\title{
Reggeon exchange from gauge/gravity duality
}

\author{
Matteo Giordano $^{a}$ and Robi Peschanski ${ }^{b}$ \\ ${ }^{a}$ Departamento de Física Teórica, Universidad de Zaragoza, \\ Calle Pedro Cerbuna 12, E-50009 Zaragoza, Spain \\ ${ }^{b}$ Institut de Physique Théorique CEA-Saclay, \\ F-91191 Gif-sur-Yvette Cedex, France \\ E-mail: giordano@unizar.es, robi.peschanski@cea.fr
}

ABSTRACT: We perform the analysis of quark-antiquark Reggeon exchange in meson-meson scattering, in the framework of the gauge/gravity correspondence in a confining background. On the gauge theory side, Reggeon exchange is described as quark-antiquark exchange in the $t$ channel between fast projectiles. The corresponding amplitude is represented in terms of Wilson loops running along the trajectories of the constituent quarks and antiquarks. The paths of the exchanged fermions are integrated over, while the "spectator" fermions are dealt with in an eikonal approximation. On the gravity side, we follow a previously proposed approach, and we evaluate the Wilson-loop expectation value by making use of gauge/gravity duality for a generic confining gauge theory. The amplitude is obtained in a saddle-point approximation through the determination near the confining horizon of a Euclidean "minimal surface with floating boundaries", i.e., by fixing the trajectories of the exchanged quark and antiquark by means of a minimisation procedure, which involves both area and length terms. After discussing, as a warm-up exercise, a simpler problem on a plane involving a soap film with floating boundaries, we solve the variational problem relevant to Reggeon exchange, in which the basic geometry is that of a helicoid. A compact expression for the Reggeon-exchange amplitude, including the effects of a small fermion mass, is then obtained through analytic continuation from Euclidean to Minkowski space-time. We find in particular a linear Regge trajectory, corresponding to a Regge-pole singularity supplemented by a logarithmic cut induced by the non-zero quark mass. The analytic continuation leads also to companion contributions, corresponding to the convolution of the same Reggeon-exchange amplitude with multiple elastic rescattering interactions between the colliding mesons.

KEYwORDS: Gauge-gravity correspondence, Nonperturbative Effects

ARXIV EPRINT: 1105.6013 


\section{Contents}

1 Introduction and summary of results $\quad 2$

2 Wilson loops and gauge/gravity duality for confining theories 6

3 Reggeon-exchange amplitude $r$

$\begin{array}{lll}3.1 & \text { Impact-parameter amplitude } & 10\end{array}$

$\begin{array}{lll}3.2 & \text { Gauge/gravity correspondence and minimal surfaces } & 13\end{array}$

$\begin{array}{lll}3.3 & \text { Saddle-point approximation } & 17\end{array}$

4 Warm-up exercise: soap film with floating boundaries $\quad 18$

5 Variational problem for the Reggeon-exchange amplitude in Euclidean space $\quad 22$

5.1 Exact solution in implicit form 23

5.2 Explicit solutions: analytical and numerical results 28

$\begin{array}{lll}5.2 .1 & \text { Case } \varphi_{0} \gg 1 & 29\end{array}$

$\begin{array}{lll}5.2 .2 \text { Case } \lambda \gg 1 & 31\end{array}$

$\begin{array}{lll}5.2 .3 & \text { Numerical results } & 32\end{array}$

6 Analytic continuation into Minkowski space-time 33

6.1 Subcritical region $b \leq b_{c} \quad 36$

6.2 Analytic continuation towards $b>b_{c} \quad 36$

$\begin{array}{llr}7 & \text { The Reggeon amplitude } & 39\end{array}$

7.1 The Reggeon singularity and small quark-mass effects 40

7.2 Prefactors 43

7.3 Multi-sheet structure of the effective action: convolution of Regge amplitudes 44

8 Conclusions and outlook $\quad 46$

$\begin{array}{ll}\text { A Evaluation of the spin factor } & 48\end{array}$

B Application to the Reggeon-exchange amplitude 51

B.1 Contraction with the bispinors 51

B.2 Evaluation of the phase factor on the solution of the saddle-point equation 53

$\begin{array}{ll}\text { C Spin-factor contribution in the large- } \lambda \text { case } & 56\end{array}$ 


\section{Introduction and summary of results}

The main difficulty in the study of hadronic soft scattering at high energy ${ }^{1}$ in the framework of Quantum Field Theory is due to the fact that it involves the nonperturbative, strong-coupling regime of the microscopic theory underlying strong interactions, namely Quantum Chromodynamics (QCD). In recent years, a remarkable achievement of the general gauge/string theories relationship (see [1] and older relevant references therein), namely the so-called gauge/gravity duality, has provided a new possible practical tool to deal with strong-coupling physics in QCD, and this has raised the hope to obtain new insights in this difficult and long-standing problem. Gauge/gravity duality, whose first precise realisation has been provided by the AdS/CFT correspondence [2-4], relates a gauge field theory at strong coupling with a dual gravity theory in the weak coupling regime, and has been the subject of intense research work over the last decade. The AdS/CFT correspondence, which is valid, strictly speaking, for the conformal $\mathcal{N}=4 \mathrm{SYM}$ gauge field theory, appears to be physically useful in the study of the high-temperature quark-gluon plasma in QCD, where the confinement property is less relevant $[5,6]$ (for a recent review see [7]). The extension of the gauge/gravity duality to non conformal confining theories is motivated by the possibility to obtain a better understanding of those nonperturbative properties of strong interactions which are sensitive to the confinement scale.

Although not yet completed, specifically for QCD, this program has shown a few properties which the gravity theory dual to QCD should have, in order to reproduce the main features of strong interactions. In particular, the presence of a confinement scale in the gauge theory translates into a characteristic scale in the gravity theory, associated for example to the horizon of a black hole [8], or to the position of a hard wall [9], or to the scale associated to a soft wall [10]. Our aim is to formulate results which may be valid in a generic confining case, independently of a specific realisation of the duality. Such an opportunity is provided by the high-energy limit of soft two-body scattering amplitudes [11-15], for which relatively general properties may be obtained where the confinement scale plays a major role.

As it is well known, from the phenomenological point of view, soft high-energy hadronhadron scattering processes can be described, in the language of Regge theory, in terms of the exchange of "families" of states between the interacting hadrons. These "families" correspond to the singularities in the complex-angular-momentum plane of the amplitude in the crossed channel (see e.g. [16]). The leading contribution at high energy comes from the so-called Pomeron, which carries the quantum numbers of the vacuum, while subleading contributions are usually called Reggeons, and correspond to various non-vacuum quantum-number exchanges. One of the aims of the theoretical study of soft high-energy scattering is to obtain an explanation of these phenomenological concepts from the underlying microscopic field theory.

While a lot of work has been done in recent years regarding the Pomeron [17-28], especially in the context of the gauge/gravity duality [11-13, 15, 29-43], the problem of Reggeon exchange has received less attention. ${ }^{2}$ In this paper we shall focus on the approach

\footnotetext{
${ }^{1} \mathrm{~A}$ soft two-body high-energy scattering process is characterised by a large center-of-mass energy squared $s$ and a small momentum transfer $t$ with respect to the typical hadronic scale, i.e., $\sqrt{s} \gg 1 \mathrm{GeV} \gtrsim \sqrt{-t}$.

${ }^{2}$ We mention however ref. [44], where a unified treatment of the signature-odd partner of the Pomeron,
} 
to $q \bar{q}$-Reggeon exchange in meson-meson scattering proposed in [14]. This approach elaborates on the formalism of [17-22], valid for soft high-energy processes, and on previous work on the use of gauge/gravity duality for scattering amplitudes [11-13]. In particular, it assumes gauge/gravity duality for a generic confining theory, and exploits the pathintegral representation for the fermion propagator [46-50] to provide an expression for the Reggeon-exchange scattering amplitude in the energy regime under investigation.

In this approach, the Reggeon-exchange amplitude is put into a relation with the expectation value of certain Euclidean Wilson loops, describing the exchange of a (Reggeised) quark-antiquark pair between the interacting hadrons. More precisely, the loop contours are made up of a fixed part, corresponding to the eikonal trajectories of the "spectator" fermions, and a "floating" part, corresponding to the trajectories of the exchanged fermions. The Reggeon-exchange scattering amplitude is obtained by summing up the contributions of these loops, through a path-integration over the trajectories of the exchanged fermions. In turn, these Wilson loops are related via the gauge/gravity duality to minimal surfaces in a curved confining metric, having the loop contour as boundary (Plateau problem), which correspond to the exchange of an open string between the interacting hadrons. Finally, the physical amplitude in Minkowski space-time is recovered by means of analytic continuation [51-57].

The determination of the relevant minimal surfaces is in general a difficult problem, and depends on the specific choice for the confining background. The key idea is to observe that an approximate solution to this problem can be found by solving a simpler Plateau problem, namely by finding a minimal surface in a flat Euclidean 4-dimensional space near the confinement scale (e.g., near a confining horizon) in the bulk. Since the precise form of the metric does not enter the simplified problem, the corresponding solution is expected to be a valid approximation independently of the specific realisation of the duality. To leading order, the amplitude is then evaluated through a saddle-point approximation of the Euclidean path-integral, which fixes the shape of the floating boundary.

The quantity to be minimised is the Euclidean "effective action" (see section 3), which consists of a linear combination of the area of the surface and of the length of its floating boundaries, and which encodes the contribution to the (Euclidean) scattering amplitude of a given shape of the floating boundary. For the sake of brevity, we will sometimes refer to our variational problem as the minimal surface problem with floating boundaries, which we will define precisely in section 3 .

It is worth noticing already at this stage an important practical aspect of this approach. For large "spectator"-quark mass (and thus large meson mass) and small exchanged-quark mass, it is argued that the relevant contributions to the path integral should come from those configurations in which the floating part of the boundary lies on a specific surface, namely the minimal surface having as boundaries two infinite straight lines. Such a surface is a helicoid, which has been already encountered in the study of soft quark-quark scattering at high energy [12].

the so-called "Odderon", and of the signature-odd Reggeons is proposed, and ref. [45], where the Regge behaviour of scattering amplitudes in QCD is obtained in an effective string approach. 
In [14], this problem was solved using a null quark mass approximation, which simplifies the minimisation procedure by reducing the effective action to the area term only, and leads to a complex solution (thus not in Euclidean space) for the saddle-point equation. ${ }^{3}$ The physical amplitude was finally obtained by means of analytic continuation of this complex solution onto a physically admissible one in Minkowski space-time. Interestingly enough, the resulting amplitude obtained in [14] was of Regge-pole type, with a linear Reggeon trajectory. Our aim in this paper is to revisit this method in a more general minimisation setting, including the length terms by considering a non-zero quark mass. In this way we find real Euclidean solutions, from which we can obtain the physical scattering amplitude in Minkowski space-time by means of a suitable analytic continuation.

In this paper, we investigate in detail and solve the Euler-Lagrange equations corresponding to the above-mentioned minimal surface problem with floating boundaries, and we discuss the properties of the resulting Reggeon-exchange amplitudes. We summarise here the main results.

- It is shown how a real solution to the minimal surface problem with floating boundaries is obtained in Euclidean space, provided the quark mass is non-zero. The minimal Euclidean "effective action" corresponding to the solution reads

$$
S_{\mathrm{eff}, \mathrm{E}}=\frac{b^{2}}{2 \pi \alpha_{\mathrm{eff}}^{\prime} \theta} f(\tilde{\varphi})+\frac{4 m b}{\theta}\left(B\left(\varphi_{0}, \tilde{\varphi}\right)-\sinh \tilde{\varphi}\right),
$$

where $1 / 2 \pi \alpha_{\text {eff }}^{\prime}$ is the string tension, $b$ is the impact-parameter distance and $\theta$ the angle between the Euclidean trajectories of the two incoming particles, and $\varphi_{0}$ and $\tilde{\varphi}$ are geometric parameters of the solution of the minimisation problem for the boundaries (see further section 5). The shape of the boundaries enters the effective action through the functions $f(\tilde{\varphi})$ and $B\left(\varphi_{0}, \tilde{\varphi}\right)$, which are obtained in an implicit form, and can be easily evaluated numerically; an explicit analytic expression is obtained in two specific regimes.

- The saddle-point equation in Euclidean space admits a real solution only in a finite region for the impact parameter $b$, namely $b \leq b_{c}=4 \pi \alpha_{\text {eff }}^{\prime} m$, and this limitation carries over to Minkowski space after analytic continuation. In order to investigate the region $b>b_{c}$, and also in order to take the massless-quark limit, we show how an analytic continuation of the result in Minkowski space to the region $b>b_{c}$ can be performed, giving rise to a sensible scattering amplitude. Our main result for the Minkowskian effective action then reads

$$
S_{\mathrm{eff}, \mathrm{M}}(s, b) \sim \frac{b^{2}}{2 \pi \alpha_{\mathrm{eff}}^{\prime} \chi} \arccos \frac{b_{c}}{b}-\frac{2 b m}{\chi} \sqrt{1-\left(\frac{b_{c}}{b}\right)^{2}}+2 \pi^{2} \alpha_{\mathrm{eff}}^{\prime} m^{2},
$$

where $\chi \sim \log s$ is substituted to $\theta$ by the analytic continuation $\theta \rightarrow-i \chi$, see section 6 .

\footnotetext{
${ }^{3}$ Quadratic fluctuations of the string around the corresponding minimal surface were also computed, leading to a constant shift in the Reggeon "intercept", i.e., the exponent of $s$ at $t=0$ in the high-energy behaviour of the amplitude [13].
} 
- Expanding eq. (1.2) for small quark mass, one finds a Gaussian-like Reggeon-exchange amplitude in impact-parameter space (up to prefactors),

$$
a(\vec{b}, \chi) \equiv \frac{i}{2 s} \int \frac{d^{2} \vec{q}}{(2 \pi)^{2}} e^{-i \vec{q} \cdot \vec{b}} \mathcal{A}_{\mathcal{R}}(s, t) \propto \exp \left\{-\frac{b^{2}}{4 \alpha_{\mathrm{eff}}^{\prime} \chi}+\frac{4 b m}{\chi}-2 \pi^{2} \alpha_{\mathrm{eff}}^{\prime} m^{2}\right\}
$$

where $\mathcal{A}_{\mathcal{R}}\left(s \sim e^{\chi}, t=-\vec{q}^{2}\right)$ is the Reggeon-exchange scattering amplitude in momentum space. The result eq. (1.3) leads to a linear Reggeon trajectory $\alpha_{\mathcal{R}}(t)=\alpha_{0}+\alpha_{\mathcal{R}}^{\prime} t$, with slope $\alpha_{\mathcal{R}}^{\prime} \equiv \alpha_{\text {eff }}^{\prime}$ equal to the inverse string tension. ${ }^{4}$ As discussed in section 7 , one is able to discuss modifications of the Regge singularity due to a small but nonzero quark mass. We find the same Regge-pole singularity obtained at $m=0$ in [14], plus a logarithmic singularity due to the non-zero quark mass. Although the nature of the Reggeon singularity is changed, the Reggeon trajectory remains linear after the inclusion of (small) quark-mass effects. Their main physical consequence is that the slope of the amplitude $\partial \mathcal{A}_{\mathcal{R}} /\left.\partial t\right|_{t=0}$ is increased, and its shrinkage with energy is strengthened.

- The analytic continuation of the Euclidean action (1.1) leads to other contributions in Minkowski space-time through the Riemann multi-sheet structure of the inverse cosine function in eq. (1.2). They take the form of a multiple convolution in momentum space of the Reggeon-exchange amplitude (1.3) with an integer number of copies of the following amplitude,

$$
\mathcal{A}_{e l}(s, t)=-4 i \pi s \int_{0}^{\infty} d b b J_{0}(q b) \exp \left\{-\frac{b^{2}}{\alpha_{\text {eff }}^{\prime} \chi}\right\} \equiv-2 i \pi s \alpha_{\text {eff }}^{\prime} \chi \exp \left\{\frac{\alpha_{\text {eff }}^{\prime} t}{4} \chi\right\}
$$

which happens to be a Regge-pole amplitude with intercept one. The properties of this amplitude allow one to interpret it as an elastic interaction between the incident mesons. Moreover, this interaction has the same features of the one obtained some time ago for dipole-dipole elastic scattering amplitudes [12]. The multiple convolution can be phenomenologically interpreted as the effect of the "rescattering corrections" to the "bare" $q \bar{q}$-Reggeon exchange.

As we have already remarked, our results for confining gauge theories are expected to be quite general, and independent of the precise realisation of the gauge/gravity duality (assuming it exists), since they rely only on general features of the dual geometry, essentially the (effective) cut-off provided by the confinement scale in the bulk. It is worth mentioning that in recent years the holographic approach has been applied also to the issue of scattering amplitudes in the context of $\mathcal{N}=4$ SYM theory, in particular using the AdS/CFT correspondence and minimal surfaces to investigate gluon-gluon elastic scattering at high energy [58]. It appears that in this case the resulting Regge trajectory is logarithmic rather than linear $[59,60]$, which is a striking difference between the predictions for conformal and confining gauge theories.

\footnotetext{
${ }^{4}$ Note that this equality is non trivial, since the string tension may be independently obtained by evaluating the confining $Q-\bar{Q}$ potential.
} 
The plan of the paper is the following. In section 2 we recall the relevant features of the gauge/gravity duality used in the evaluation of Wilson-loop expectation values in a confining theory. In section 3 we review the approach of [14] to Reggeon exchange, discussing in some detail the approximations involved and the Euler-Lagrange equations for the relevant minimal surface with floating boundaries in the presence of a non-zero quark mass. In section 4 we solve explicitly the equations in a planar case, which happens to be related to a classical problem involving a soap film with floating boundaries. In section 5 we solve the Euler-Lagrange equations related to the Reggeon-exchange problem, where the basic geometry is that of a helicoid. We discuss in particular the issue of smoothness conditions, and we obtain an exact solution in implicit form for the general case. We then investigate analytically two limits of the solution, in which we are able to write it down explicitly and to uncover the dependence on the relevant variables. We also compare them with some numerical results for the exact solution. In section 6 we perform the analytic continuation into Minkowski space-time, and discuss the properties of the resulting Reggeon amplitude, in particular regarding the dependence on the energy and on the impact parameter. In section 7 we discuss the effect of a non-zero quark mass on the nature of the Reggeon singularity and on the Reggeon trajectory. We also discuss the other companion contributions to the amplitude coming from the multi-sheet structure of the Minkowskian effective action. Finally, in section 8 we draw our conclusions and show some prospects for the future. A few technical details are given in the appendices.

\section{Wilson loops and gauge/gravity duality for confining theories}

In this section we recall the relevant aspects of the gauge/gravity duality which will be used in the following. We begin with the now standard AdS/CFT correspondence [2-4], which relates type IIB string theory in $A d S_{5} \times S^{5}$ in the weak-coupling, supergravity limit, to four-dimensional $\mathcal{N}=4 \mathrm{SYM}$ theory, which is a conformal and non confining field theory, in the limit of large number of colours $N_{c}$ and strong 't Hooft coupling $\lambda=g_{\mathrm{YM}}^{2} N_{c}$, where $g_{\mathrm{YM}}$ is the coupling constant in the gauge theory. Expectation values in the field theory can be obtained from the dual gravity theory with the appropriate prescription; for further convenience, we focus on the problem of the vacuum expectation value of Wilson loops. Going over to Euclidean signature, the prescription for a Wilson loop running along the path $\mathcal{C}$ is given by the following area law [61-64],

$$
\langle\mathcal{W}[\mathcal{C}]\rangle \sim \mathcal{F}[\mathcal{C}] e^{-\frac{1}{2 \pi \alpha^{\prime}} A_{\min }[\mathcal{C}]} .
$$

Here $A_{\min }[\mathcal{C}]$ is the area of a minimal surface in the Euclidean version of the $A d S_{5}$ metric, which is obtained from the original metric

$$
d s_{\text {AdS }}^{2}=\frac{d z^{2}}{z^{2}}+\frac{\eta_{\mu \nu} d x^{\mu} d x^{\nu}}{z^{2}}
$$

where $\eta_{\mu \nu}$ is the four-dimensional Minkowski metric $(\mu, \nu=0, \ldots, 3)$, by replacing $\eta_{\mu \nu} \rightarrow$ $\delta_{\mu \nu}$. The minimal surface has as boundary the contour $\mathcal{C}$ at $z=0$, i.e., on the fourdimensional boundary of $A d S_{5}$; moreover, $\mathcal{F}$ is a prefactor due to quantum fluctuations around the minimal surface, and $1 / 2 \pi \alpha^{\prime}$ is the string tension. 
In order to extend the duality to the confining case, one has to properly modify the background metric in the dual gravity theory, taking into account that the theory is no more conformal. Although the precise realisation of the duality (assuming it exists) is not known yet, a common feature of various attempts to describe a confining theory in terms of a gravity dual is the presence of a characteristic scale $R_{0}$ in the metric, which separates the small and large $z$ regions. With the appropriate choice of coordinates, while for small $z$ the metric diverges as some inverse power of $z$, for $z$ of the order of $R_{0}$ it turns out to be effectively flat. The interpretation in the dual confining field theory is that the scale $R_{0}$ provides the confinement scale. For example, in the case of the AdS/BH metric of [8], such a scale is provided by the position in the fifth dimension of the black-hole horizon. The relevant part of the metric reads

$$
d s_{\mathrm{AdS} / \mathrm{BH}}^{2}=\frac{16}{9} \frac{1}{f(z)} \frac{d z^{2}}{z^{2}}+\frac{\eta_{\mu \nu} d x^{\mu} d x^{\nu}}{z^{2}}+\ldots,
$$

where $f(z)=z^{2 / 3}\left(1-\left(z / R_{0}\right)^{4}\right)$. The near-horizon geometry is effectively flat,

$$
d s_{\mathrm{hor}}^{2} \simeq \frac{1}{R_{0}^{2}} \eta_{\mu \nu} d x^{\mu} d x^{\nu}
$$

The prescription used to calculate a Wilson loop expectation value (in Euclidean signature) is the same as above, but substituting the AdS metric with an appropriate confining background [65-67]. Also, one has to replace $1 / 2 \pi \alpha^{\prime}$ with an effective string tension $1 / 2 \pi \alpha_{\text {eff }}^{\prime}$, which depends on the particular background metric: in the case of the AdS/BH metric (2.3), for example, it is given by $1 / 2 \pi \alpha_{\mathrm{eff}}^{\prime}=\sqrt{2 g_{\mathrm{YM}}^{2} N_{c}} / 2 \pi R_{0}^{2}$. Although it is not possible to determine its explicit expression in the general case, $\alpha_{\text {eff }}^{\prime}$ can be determined phenomenologically by comparison with the heavy quark-antiquark confining potential $V_{Q \bar{Q}}(R)=\left(1 / 2 \pi \alpha_{\text {eff }}^{\prime}\right) R$.

The analytic solution of the Plateau problem ${ }^{5}$ is a highly non trivial task already in flat Euclidean space, and it is even harder in a non-flat metric such as (2.3): some approximations are then necessary in order to obtain an analytic expression. A reasonable and manageable scheme is obtained by means of a near-horizon approximation, taking into account the above-mentioned features which the dual gravity theory is expected to have $[12,14]$ (see also $[66,67]$ ). The small- $z$ behaviour suggests that, in order to minimise the area, it is convenient for the surface to rise almost vertically from the boundary, without appreciable motion in the other directions, at least when the typical size $b$ of the Wilson loop is not too small, ${ }^{6}$ see figure 1 (left). On the other hand, the geometry of the surface is different for smaller values of $b$, see figure 1 (right). The presence of a horizon puts an upper bound on this vertical rise; moreover, when $z \sim R_{0}$, the surface lives effectively in flat space. As a result, the minimal surface is expected to be constituted by two parts: an almost vertical wall rising from the boundary up to the horizon, and transporting there the boundary conditions, and a solution of the Plateau problem in flat space.

\footnotetext{
${ }^{5}$ An analytic solution is required for performing the continuation from Euclidean to Minkowski space.

${ }^{6}$ This approximation is expected to be valid when $b$ is greater than $R_{0}$, which should correspond to the distance at which the interquark potential becomes linear.
} 


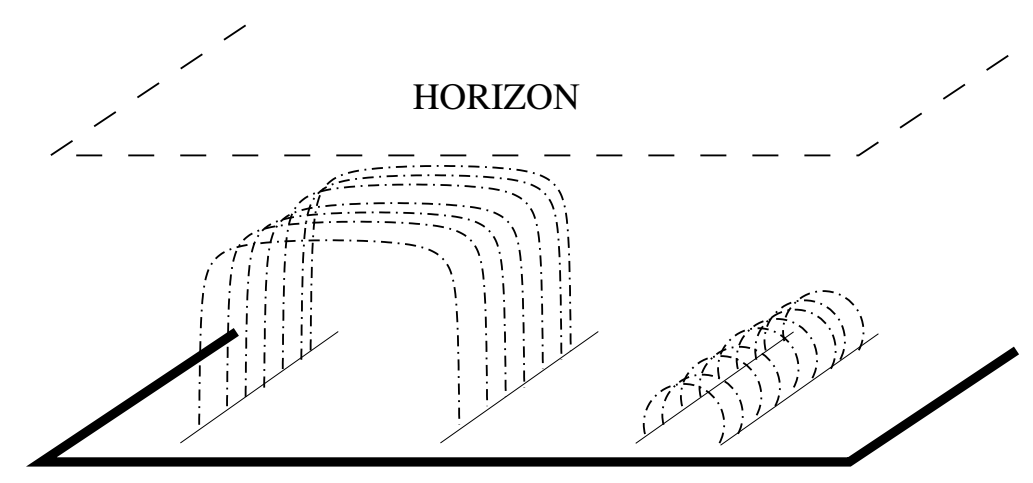

Boundary

Figure 1. Minimal surface in the confining black-hole geometry. For simplicity, the Wilson lines are drawn here with vanishing angle of tilt $\theta=0$. For large enough impact parameter (left), the minimal surface rises as a vertical wall from the boundary, and is almost flat near the horizon. At small impact parameter (right) the surface is more similar to a non confining case. The picture is taken from [12].

A schematic representation of this geometrical configuration in the bulk for parallel Wilson lines, relevant to the determination of the confining potential, is displayed in figure 1 (left). The area of the vertical wall is divergent, but in the expression for scattering amplitudes it is usually cancelled by appropriate normalisation factors. ${ }^{7}$ The problem is then reduced to a calculation in four-dimensional Euclidean space: this is reminiscent of the old "QCD string" approach (see [68] for a comprehensive review), although in this case it should be the result of an approximation to a higher-dimensional critical string theory, and thus it should not suffer the problems of the old approach.

Due to the generality of the geometrical picture leading to the considered flat-space approximation near the confinement scale, analogous to a near-horizon approximation, one expects to get results valid for a gauge/gravity duality for a generic confining gauge field theory, and hopefully for QCD assuming the existence of its yet unknown gravity dual. We shall now turn to the determination of a Reggeon-exchange amplitude in this context.

\section{Reggeon-exchange amplitude}

In this section we recall the method of ref. [14] for the determination of the Reggeonexchange contribution to the meson-meson scattering amplitude in the soft high-energy regime, developing on a few points which are relevant for our analysis and more briefly discussed in that work.

The starting point is to adopt a description of the interacting hadrons in terms of their constituent partons. Such an approach to soft high energy hadron-hadron scattering has been introduced in [17], where it was used, together with an LSZ reduction scheme

\footnotetext{
${ }^{7}$ This is the case, for example, for the dipole-dipole scattering amplitude expressed in terms of Wilsonloop correlation functions [12]: here the relevant size is the distance $b$ between the two loops, which play the role of disconnected boundary for the minimal surface, and the normalisation factor is the product of the Wilson-loop expectation values.
} 
and an eikonal approximation for the propagators, in order to derive approximate nonperturbative formulas for the scattering amplitudes. The basic idea is that the leading Pomeron-exchange contribution to the elastic amplitude comes from processes which are elastic and soft at the level of the constituent partons, justifying an eikonal-like approach. In a space-time picture of these processes, the partons travel along their classical, straightline trajectories, exchanging only soft gluons which leave these trajectories practically unperturbed. This approach to the Pomeron-exchange amplitude has been investigated and extended in a number of papers [11-13, 15, 18-28].

In particular, in the case of meson-meson scattering, one can describe the mesons, in a first approximation, in terms of a wave packet of transverse colourless quark-antiquark dipoles $[18,20,21,24]$. The mesonic scattering amplitude is reconstructed, after folding with the appropriate wave functions, from the scattering amplitude of such dipoles. Since here we are interested only in the Reggeon trajectory, which, being a universal quantity, should not depend on the details of the meson wave function, we can focus on the dipoledipole amplitude, which is expected to encode the relevant features of the process. Stated differently, invoking the universality of Reggeon exchange, one can consider mesons whose wave function is strongly peaked around some average value $|\vec{R}|$ of the dipole size.

Using this simplified description for the mesons, Reggeon exchange is identified as an inelastic process at the partonic level, involving the exchange of a quark-antiquark pair between the colliding dipoles. More precisely, the corresponding space-time picture is the following (see figure 2). Before and after the interaction time (which may be long for a soft interaction), the partons inside the high-energy mesons travel approximately along their classical, straight-line (eikonal) trajectories. During the interaction time, a pair of valence partons is exchanged in the $t$ channel between the mesons, and thus their trajectories bend, connecting the incoming and outgoing eikonal trajectories; the other partons exchange only soft gluons, and their straight-line trajectories are left practically undisturbed. The softness of the process requires that the exchanged fermions carry a small fraction of longitudinal momentum of the mesons. ${ }^{8}$

In order to avoid inessential complications, we consider the scattering of two heavylight mesons $M_{1,2}$ of large mass $m_{1,2}$, i.e., $M_{1}=Q \bar{q}$ and $M_{2}=\bar{Q}^{\prime} q$, where $Q$ and $\bar{Q}^{\prime}$ are heavy and of different flavours, while $q$ and $\bar{q}$ are light and of the same flavour. In this way the total scattering amplitude amounts to a single type ${ }^{9}$ of Reggeon-exchange process, namely the one in which $q$ and $\bar{q}$ are exchanged in the $t$ channel, plus the Pomeronexchange component, where there are no exchanged fermions. Moreover, the choice of heavy mesons is made in order for the typical size of the dipoles to be small, since in this case $\left|\vec{R}_{1,2}\right| \sim m_{1,2}^{-1} \ll \Lambda_{\mathrm{QCD}}^{-1}$; the reasons for this choice will be explained later on.

\footnotetext{
${ }^{8} \mathrm{~A}$ rigorous quantitative formulation of this statement is still lacking and requires a more detailed study of Reggeon exchange from first principles [69].

${ }^{9}$ For physical mesonic amplitudes, different Regge trajectories are introduced depending on the exchanged quantum numbers and the quark flavours. In the present case, we consider only the simplest case with only light-quark exchange. For completion however, as we will discuss in section 7, the Reggeon exchange is not isolated. It is expected to be accompanied by contributions corresponding to the so-called "rescattering corrections", which we will also obtain from holography.
} 


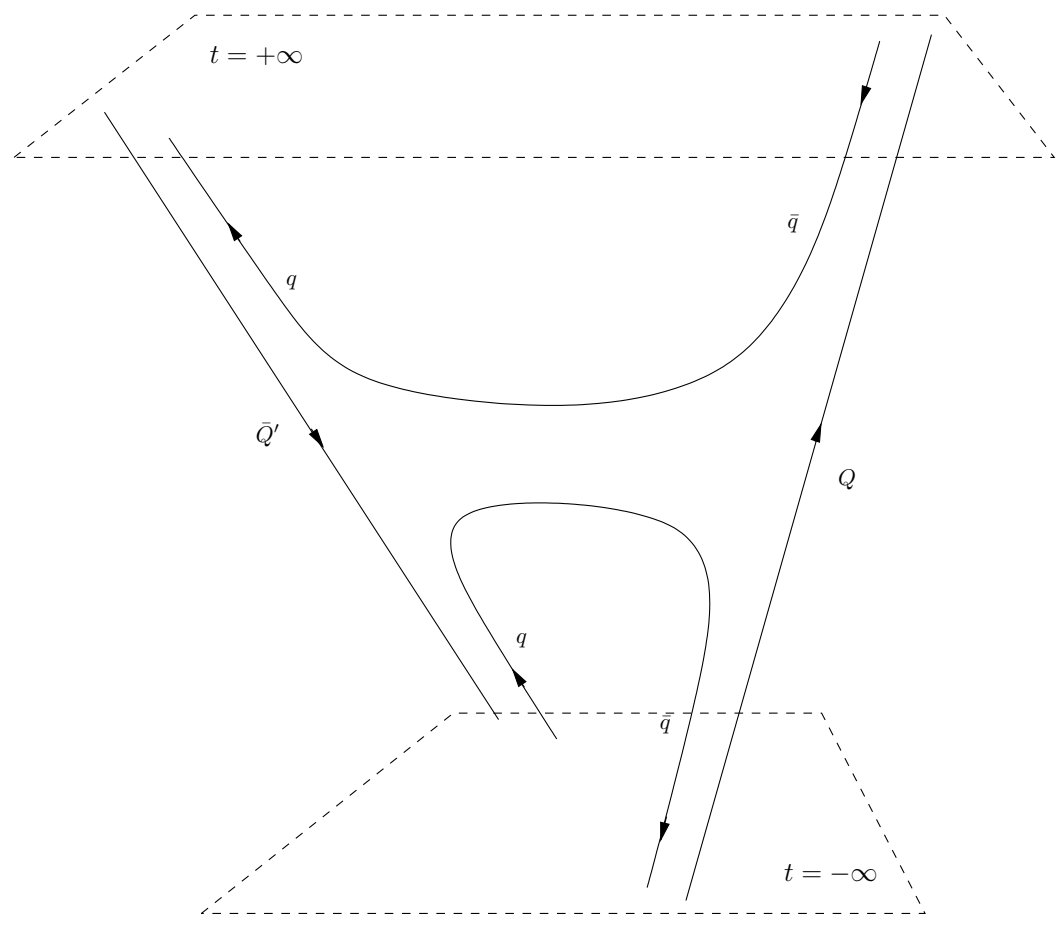

Figure 2. Space-time picture of the Reggeon-exchange process. $Q, \bar{Q}^{\prime}$ are heavy and fast quark and antiquark, which follow straight-line trajectories in the eikonal approximation. $q, \bar{q}$ are the exchanged light quark and antiquark, describing the Reggeon exchange between the incident $Q \bar{q}$ and $\bar{Q}^{\prime} q$ mesons (see text).

\subsection{Impact-parameter amplitude}

At this point, let us describe in some detail the expression for the Reggeon-exchange contribution $\mathcal{A}_{\mathcal{R}}(s, t)$ to the scattering amplitude proposed in [14]. To this extent, let us introduce the impact-parameter amplitude $a(\vec{b}, \chi)$,

$$
\mathcal{A}_{\mathcal{R}}(s, t)=-i 2 s \int d^{2} b e^{i \vec{q} \cdot \vec{b}} a(\vec{b}, \chi),
$$

where $\chi$ is the hyperbolic angle between the classical trajectories of the colliding mesons, related to the center-of-mass energy squared $s$ through $\chi \simeq \log s /\left(m_{1} m_{2}\right)$ (for $s \rightarrow \infty$ ), with $m_{1,2}$ the masses of the mesons, and $t=-\vec{q}^{2}$. Here we do not write explicitly the dependence on the orientation of the dipoles. According to the space-time picture of the process given above, the eikonal approximation can no longer be used to describe the propagation of the light quarks, and different techniques are required. Working in Euclidean space, the authors of [14] exploit the path-integral representation for the fermion propagator in an external non-Abelian gauge field [46-50], in order to write down a Euclidean "amplitude" $\tilde{a}(\vec{b}, \theta, T)$ in terms of a path-integral over the trajectories of the light quarks. Here $\theta$ is the angle between the Euclidean trajectories of the mesons, and $T$ is an IR cutoff, which will be explained shortly. The physical Minkowskian amplitude $a(\vec{b}, \chi)$ in eq.(3.1) is finally recovered by means of the analytic continuation $\theta \rightarrow-i \chi, T \rightarrow i T$ [51-57], contracting with the appropriate Dirac spinors for the quarks and antiquarks, and removing the IR 
cutoff by taking the limit $T \rightarrow \infty$ :

$$
\begin{aligned}
a(\vec{b}, \chi)= & \lim _{T \rightarrow \infty}[\tilde{a}(\vec{b},-i \chi, i T)]_{\alpha^{\prime} \beta^{\prime} \gamma^{\prime} \delta^{\prime} ; \alpha \beta \gamma \delta} \bar{u}_{\alpha^{\prime}}^{\left(s_{Q}^{\prime}\right)}\left(p_{Q}^{\prime}\right) u_{\alpha}^{\left(s_{Q}\right)}\left(p_{Q}\right) v_{\beta^{\prime}}^{\left(t_{\bar{Q}^{\prime}}^{\prime}\right)}\left(p_{\bar{Q}^{\prime}}^{\prime}\right) \bar{v}_{\beta}^{\left(t_{\bar{Q}^{\prime}}\right)}\left(p_{\bar{Q}^{\prime}}\right) \\
& \times \bar{v}_{\gamma}^{\left(t_{\bar{q}}\right)}\left(p_{\bar{q}}\right) u_{\delta}^{\left(s_{q}\right)}\left(p_{q}\right) v_{\gamma^{\prime}}^{\left(t_{\bar{q}}^{\prime}\right)}\left(p_{\bar{q}}^{\prime}\right) \bar{u}_{\delta^{\prime}}^{\left(s_{q}^{\prime}\right)}\left(p_{q}^{\prime}\right) .
\end{aligned}
$$

Even in the simplified setting that we are considering here, in order to reconstruct the mesonic amplitudes one has still to average over the orientation of the dipoles; moreover, one should also contract the spin indices with the appropriate wave functions. As we have already said, we are interested here only in the Reggeon trajectory, and so the detailed dependence on spin should not be relevant, and it will not be discussed in this work. As for the dependence on the orientation, the choice of large meson masses, or equivalently of small dipole sizes, will make it negligible in a first approximation, as it will be discussed further on.

All in all, the Euclidean "amplitude" $\tilde{a}(\vec{b}, \theta, T)$, which should encode the features of the Reggeon trajectory, can be written symbolically as

$$
\tilde{a}(\vec{b}, \theta, T)=\mathcal{Z}^{-1} \int \mathcal{D} \mathcal{C}_{+} \mathcal{D C}-\langle\mathcal{W}[\mathcal{C}]\rangle e^{-m_{0} L[\mathcal{C}]} \mathcal{I}[\mathcal{C}],
$$

where the different terms $\langle\mathcal{W}[\mathcal{C}]\rangle, L[\mathcal{C}], \mathcal{I}[\mathcal{C}], \mathcal{Z}$ are defined as follows.

- $\langle\mathcal{W}[\mathcal{C}]\rangle$ is the expectation value of the Euclidean Wilson loop running along the path $\mathcal{C}$ (see figure 3), composed essentially of the Euclidean trajectories of the partons,

$$
\begin{aligned}
\mathcal{C} & =\mathcal{C}_{1} \circ \mathcal{S}_{1}^{-} \circ \mathcal{C}_{+} \circ \mathcal{S}_{2}^{-} \circ \mathcal{C}_{2} \circ \mathcal{S}_{2}^{+} \circ \mathcal{C}_{-} \circ \mathcal{S}_{1}^{+}, \\
\mathcal{W}[\mathcal{C}] & =\operatorname{tr}\left\{W\left[\mathcal{C}_{1}\right] W\left[\mathcal{S}_{1}^{-}\right] W\left[\mathcal{C}_{-}\right] W\left[\mathcal{S}_{2}^{-}\right] W\left[\mathcal{C}_{2}\right] W\left[\mathcal{S}_{2}^{+}\right] W\left[\mathcal{C}_{+}\right] W\left[\mathcal{S}_{1}^{+}\right]\right\},
\end{aligned}
$$

where $W\left[\mathcal{C}_{i}\right]$ is the Wilson line along the path $\mathcal{C}_{i}$.

More precisely, $\mathcal{C}_{1}$ and $\mathcal{C}_{2}$ are the straight-line paths corresponding to the heavy partons $Q$ and $\bar{Q}^{\prime}$, respectively, which are fixed, ${ }^{10}$

$$
\begin{aligned}
\mathcal{C}_{1}: X_{1}(\nu) & =u_{1} \nu+\frac{b}{2}+\frac{R_{1}}{2}, & & \nu \in[-T, T], \\
\mathcal{C}_{2}: X_{2}(\nu) & =-u_{2} \nu-\frac{b}{2}-\frac{R_{2}}{2}, & \nu & \in[-T, T], \\
u_{1} & =\left(\cos \frac{\theta}{2}, \sin \frac{\theta}{2}, \overrightarrow{0}\right), & u_{2} & =\left(\cos \frac{\theta}{2},-\sin \frac{\theta}{2}, \overrightarrow{0}\right), \\
b & =(0,0, \vec{b}), & R_{i} & =\left(0,0, \vec{R}_{i}\right),
\end{aligned}
$$

while $\mathcal{C}_{+,-}$are the curved paths corresponding to the exchanged light partons, which have to be integrated over,

$$
\begin{array}{rlrl}
\mathcal{C}_{+}: X_{+}(\nu), \dot{X}_{+}^{2}(\nu) & =1, & \nu & \in\left[0, L_{+}\right], \\
X_{+}(0) & =u_{1} T+\frac{b}{2}-\frac{R_{1}}{2}, & X_{+}\left(L_{+}\right) & =u_{2} T-\frac{b}{2}+\frac{R_{2}}{2}, \\
\nu & \in\left[0, L_{-}\right], \\
\mathcal{C}_{-}: X_{-}(\nu), \dot{X}_{-}^{2}(\nu) & =1, & & \\
X_{-}(0) & =-u_{2} T-\frac{b}{2}+\frac{R_{2}}{2}, X_{-}\left(L_{-}\right)=-u_{1} T+\frac{b}{2}-\frac{R_{1}}{2},
\end{array}
$$

\footnotetext{
${ }^{10}$ Here and in the following we denote with $\vec{v}$ a two-dimensional vector. The components of the Euclidean vectors are chosen to be $x=\left(x_{4}, x_{1}, \vec{x}\right)$ with $\vec{x}=\left(x_{2}, x_{3}\right)$.
} 


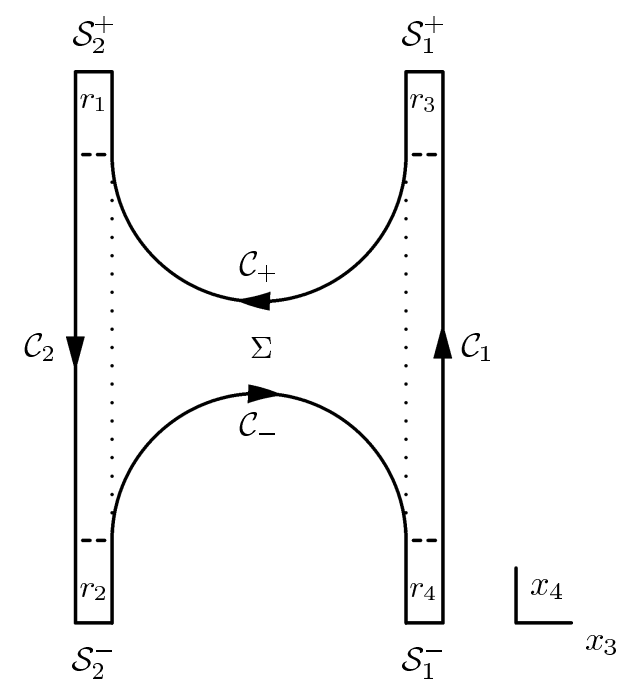

Figure 3. Schematic representation of the Wilson loop contour relevant to Reggeon exchange. The "tilted" contour (solid line) is projected on the $\left(x_{4}-x_{3}\right)$ plane for simplicity. The dashed lines delimit the various regions of the corresponding minimal surface, to be discussed below. The dotted lines correspond to the "virtual" eikonal trajectories of the light quarks, which together with $\mathcal{C}_{1,2}$ describe the free propagation of the mesons.

and $\mathcal{S}_{1,2}^{ \pm}$are straight-line paths in the transverse plane (see figure 3 ), connecting the four pieces above, which are introduced in order to make the whole expression gaugeinvariant. The path-integration over the exchanged-quark trajectories $\mathcal{C}_{ \pm}$is denoted simbolically by $\int \mathcal{D C} \mathcal{C}_{ \pm}$.

In the expressions above, the condition $\dot{X}_{i}^{2}=1$ makes of $\nu$ the natural parameter along the curve: this condition comes from the integration over momenta in the path integral for the Euclidean fermion propagator [49, 50]. As we have already said, the sizes $\left|\vec{R}_{i}\right|$ of the dipoles are of the order of the (small) inverse mass of the mesons, $\left|\vec{R}_{i}\right| \sim m_{i}^{-1}$.

- $L[\mathcal{C}]$ is the length of the path traveled by the light quarks,

$$
L[\mathcal{C}] \equiv L\left[\mathcal{C}_{+}\right]+L\left[\mathcal{C}_{-}\right]=L_{+}+L_{-},
$$

and $m_{0}$ is the (bare) mass of the light quark. As we will see below in more detail, the length-term factor $e^{-m_{0} L[\mathcal{C}]}$ in eq. (3.3) plays an important stabilisation role in the minimisation procedure related to the saddle-point approximation of the pathintegral.

- $\mathcal{I}[\mathcal{C}] \equiv \otimes_{i=\{1,2,+,-\}} \mathcal{I}_{i}\left[\mathcal{C}_{i}\right]$ is the product of the spin factors $[49,50]$ corresponding to the various fermionic trajectories, and it comes from the integration over momenta in the path-integral representation for the fermion propagator. Its subfactors are given 
by the path-ordered products

$$
\mathcal{I}_{i}\left[\mathcal{C}_{i}\right]=\prod_{X_{i}(\nu) \in \mathcal{C}_{i}} \frac{1+\dot{X}_{i}(\nu)}{2},
$$

where we have used the notation $\not x \equiv x_{\mu} \gamma_{E \mu}$, with $\gamma_{E \mu}$ the Euclidean Dirac matrices (see appendix A), and where we understand that terms corresponding to larger values of the parameter $\nu$ appear on the left.

- $\mathcal{Z}$ is a normalisation constant, which was implicitly assumed in [14], whose role is to make the amplitude IR-finite. In principle, one should be able to determine it from first principles; at the present stage, we adopt a more pragmatic approach, fixing it "by hand" in order to remove infrared divergences.

Before proceeding, a comment is in order. Although there are reasonable arguments for the validity of eq. (3.3) as a nonperturbative, approximate expression for the Reggeonexchange amplitude [14], a direct derivation of it from first principles is not yet known, contrary to the Pomeron-exchange case. Also, the analytic continuation used to obtain the Minkowskian amplitude has been proved to be the correct one in the case of the Pomeronexchange amplitude: there is not yet an explicit proof that it is the correct one also in the case of Reggeon exchange, although it seems quite plausible. These two issues are currently under investigation, ${ }^{11}$ and since the approach described in this section appears to be basically correct, we will use it as the starting point for our analysis.

\subsection{Gauge/gravity correspondence and minimal surfaces}

The following step is the application of the gauge/gravity correspondence, which, as discussed in section 2, allows to write the Wilson-loop expectation value as

$$
\langle\mathcal{W}[\mathcal{C}]\rangle=\mathcal{F}[\mathcal{C}] e^{-\frac{1}{2 \pi \alpha_{\text {eff }}^{\prime}} A_{\min }[\mathcal{C}]},
$$

where $A_{\min }[\mathcal{C}]$ is the area of the minimal surface having the contour $\mathcal{C}$ as boundary, and $\mathcal{F}[\mathcal{C}]$ contains the contributions of fluctuations around this surface. In this work we stick to the "quenched" approximation, ${ }^{12}$ while loop corrections will be considered in a future work [71].

At this point, one should in principle solve the Plateau problem in a curved background for a general boundary, and then integrate over all possible boundaries: this is a formidable task, which is currently out of reach. In order to simplify the problem, it is useful to recall the physical picture of the process, already discussed above, and sketched in figure 2. Before

\footnotetext{
${ }^{11} \mathrm{~A}$ first analysis indicates that the basic formula is essentially correct, apart from minor modifications which do not change the results on the intercept and the slope of the Reggeon trajectory; moreover, the analytic continuation required to obtain the physical amplitude turns out to be essentially the same as in the Pomeron-exchange case. A detailed study of these issues is delayed to a future publication [69].

${ }^{12}$ Expression (3.9) does not contain the effect of dynamical fermions, which are subleading at large $N_{c}$. A way to include such effects, going beyond the "quenched" approximation, has been suggested in [70], making use of the world-line formalism to express the fermion-matrix determinant as a sum of Wilson loops over all possible contours.
} 
and after the interaction, the partons travel along their eikonal, straight-line trajectories, and during the time of interaction the light quarks are exchanged between the two mesons. Translating this picture to Euclidean space, we then expect that the main contributions to the path integral come from those paths $\mathcal{C}_{ \pm}$which away from the central (interaction) region are straight lines, coinciding with the eikonal trajectories of the light quarks. As a consequence, the relevant minimal surfaces are essentially made up of a central strip (corresponding to region $\Sigma$ in figure 3), bounded by the curved part of the light-quark trajectories, which corresponds to the exchanged Reggeon, and four rectangles (regions $r_{1,2,3,4}$ in figure 3), corresponding to the free propagation of mesons before and after the interaction.

In the case that we are considering, namely small dipole sizes corresponding to heavy mesons, the part of the minimal surface corresponding to these rectangles is determined by the near-boundary behaviour of the metric, reaching a maximal value $z_{\max } \sim \mathcal{O}\left(\left|\vec{R}_{1,2}\right|\right)$ in the $z$-direction, and thus not feeling the confinement scale, see figure 1 (right). On the other hand, for large enough $b$, in the central region we can use the approximation scheme discussed in section 2. In this region the minimal surface is expected to be made up of an almost vertical wall of area $A_{\text {wall }}$, extending from the boundary of AdS up to the region where the metric is effectively flat (e.g., the black-hole horizon of ref. [8]), and a minimal surface living in the effectively flat metric, bounded by the light-quark trajectories transported from the boundary of AdS to the effectively flat region, see figure 1 (left).

Within this configuration, the geometry of the flat part of the Reggeon strip is governed by the (almost) infinite straight lines corresponding to the eikonal trajectories of the heavy quarks, transported to the effectively flat region. This suggests that the relevant contributions come from configurations in which the floating boundaries lie on the corresponding helicoid. Indeed, the helicoid has been recognised as the minimal surface associated with soft elastic quark-quark (and also quark-antiquark) scattering at high energy [12]. This assumption is expected to be sensible only for small quark mass (more precisely for small constituent quark mass, see footnote 20), as we will discuss further on. We then recover the same basic geometry already found in the treatment of Pomeron exchange, the difference being the presence of partially floating, instead of fully fixed boundaries.

Notice that since we are considering the case $\left|\vec{R}_{1,2}\right| \ll b$, we can neglect the size of the dipole in the interaction region, so that the eikonal trajectories of the light and heavy quarks coincide at the given level of approximation. Therefore, to first order the flat part of the "strip" $\Sigma$ takes the form

$$
\begin{aligned}
& X^{\text {hel }}(\bar{\tau}, \sigma)=\left(\cos \left(\frac{\theta \sigma}{b}\right) \bar{\tau}, \sin \left(\frac{\theta \sigma}{b}\right) \bar{\tau}, \frac{\vec{b}}{b} \sigma\right), \\
& \sigma \in[-b / 2, b / 2], \quad \bar{\tau} \in\left[-\tau^{-}(\sigma), \tau^{+}(\sigma)\right], \quad \tau^{ \pm}(\sigma) \geq 0 .
\end{aligned}
$$

The path-integral is then reduced to the integration over the curved part of the lightquark trajectories, constrained now to lie on the helicoid, i.e., over the "profiles" $\tau^{ \pm}(\sigma)$ which constitute the boundary of the relevant piece of helicoid; the remaining parts of the paths $\mathcal{C}_{ \pm}$lie on the eikonal light-quark trajectories. Notice that for any choice of $\tau(\sigma)$ in eq. (3.10), the resulting surface is automatically a minimal surface in flat space, i.e., a surface with zero mean curvature. 
The remaining part of the minimal surface is made up of the vertical wall and of the four rectangles. In turn, the vertical wall is made of four pieces, corresponding to the paths $\mathcal{C}_{ \pm}$and to those pieces of the paths $\mathcal{C}_{1,2}$ bounding the interaction region (i.e., between the dashed lines in figure 3 ). The rectangles are deformed in the region where they connect to the interaction region (near the dashed lines in figure 3), where the surface rises steeply to the effectively flat region; nevertheless, the area of these regions is proportional to $\left|\vec{R}_{1,2}\right|$, and can be neglected.

In this approximation, the dependence of the minimal surface on the orientation of the dipoles is trivial, as anticipated. Moreover, in this case the spin factor simplifies considerably, and it can be explicitly evaluated. ${ }^{13}$ The details of the calculation are given in appendix $\mathrm{A}$, here we quote only the final result,

$$
\mathcal{I}\left[\mathcal{C}_{i}\right]=\mathcal{N}_{i} P\left(\dot{X}_{i}\left(\nu_{f}\right)\right) \mathcal{U}_{i} P\left(\dot{X}_{i}\left(\nu_{i}\right)\right),
$$

where the various quantities are defined as follows,

$$
\begin{aligned}
P(n) & =\frac{1+\not h}{2}, \quad \mathcal{N}_{i}=\left(\frac{1+\dot{X}_{i}\left(\nu_{f}\right) \cdot \dot{X}_{i}\left(\nu_{i}\right)}{2}\right)^{-\frac{1}{2}}, \\
\mathcal{U}_{i} & =\operatorname{diag}\left(e^{-\frac{i}{2} \Phi\left(\mathcal{C}_{\vec{u}_{i}}\right)}, e^{\frac{i}{2} \Phi\left(\mathcal{C}_{\vec{u}_{i}}\right)}, e^{\frac{i}{2} \Phi\left(\mathcal{C}_{\vec{u}_{i}}\right)}, e^{-\frac{i}{2} \Phi\left(\mathcal{C}_{\vec{u}_{i}}\right)}\right) .
\end{aligned}
$$

Here $X_{i}\left(\nu_{i, f}\right)$ are the initial and final points of $\mathcal{C}_{i}$, and the (real) phases $\Phi\left(\mathcal{C}_{\vec{u}_{i}}\right)$, which depend on the shape of the path, are given in appendix A.

Since the paths $X_{1,2}$ are fixed straight lines, and moreover, for the relevant paths, $X_{ \pm}$lie on the eikonal trajectories of the light quarks near the initial and final points, it is possible to factor out of the path integral the quantities $\mathcal{N} \equiv \prod_{i=\{1,2,+,-\}} \mathcal{N}_{i}$ and $\Omega^{(i, f)} \equiv \otimes_{i=\{1,2,+,-\}} P\left(\dot{X}_{i}\left(\nu_{i, f}\right)\right)$. Denoting in short $\mathcal{U}=\otimes_{i=\{1,2,+,-\}} \mathcal{U}_{i}$, we have

$$
\begin{aligned}
\tilde{a}(\vec{b}, \theta, T) \sim \mathcal{Z}^{-1} \mathcal{N} \Omega^{(f)} & \left\{\int \mathcal{D} \tau^{+} \mathcal{D} \tau^{-} \mathcal{F}\left[\tau^{+}, \tau^{-}\right] e^{-\frac{1}{2 \pi \alpha_{\text {eff }}^{\prime}}\left\{A_{\text {min }}^{\text {hel }}\left[\tau^{+}, \tau^{-}\right]+A_{\text {rect }}+A_{\text {wall }}\right\}}\right. \\
& \left.\times e^{-m_{0}\left\{L^{\text {hel }}\left[\tau^{-}\right]+L^{\text {hel }[}\left[\tau^{+}\right]+4 T-L_{0}\left[\tau^{-}\right]-L_{0}\left[\tau^{+}\right]\right\}} \mathcal{U}\left[\tau^{+}, \tau^{-}\right]\right\} \Omega^{(i)}
\end{aligned}
$$

where the appropriate contraction of indices among $\Omega^{(f)}, \mathcal{U}$ and $\Omega^{(i)}$ is understood, and we have made explicit the dependence of $\mathcal{U}$ on $\tau^{ \pm}$. The area $A_{\text {min }}^{\text {hel }}$ of the helicoidal "Reggeon strip", and the length $L^{\text {hel }}$ of the boundaries, can now be written explicitly as functionals of $\tau^{ \pm}(\sigma)$,

$$
\begin{aligned}
A_{\min }^{\mathrm{hel}}\left[\tau^{+}, \tau^{-}\right] & =\int_{-\frac{b}{2}}^{+\frac{b}{2}} d \sigma \int_{-\tau^{-}(\sigma)}^{+\tau^{+}(\sigma)} d x \sqrt{1+(p x)^{2}} \\
L^{\mathrm{hel}}\left[\tau^{ \pm}(\sigma)\right] & =\int_{-\frac{b}{2}}^{+\frac{b}{2}} d \sigma \sqrt{1+\left(p \tau^{ \pm}(\sigma)\right)^{2}+\left(\dot{\tau}^{ \pm}(\sigma)\right)^{2}} .
\end{aligned}
$$

where we have used the notation

$$
p=\theta / b .
$$

\footnotetext{
${ }^{13}$ The first calculation of ref. [14] has been redone with a different result, see appendix A.
} 
In (3.13), the contributions $A_{\text {rect }}$ and $A_{\text {wall }}$ correspond to the four rectangles $r_{1-4}$ (see figure 3 ) and to the vertical wall, respectively. Moreover, $2 T-L_{0}\left[\tau^{ \pm}\right]$is the length of the straight-line part of the light-quark trajectories, with $L_{0}$ depending only on the endpoints,

$$
L_{0}\left[\tau^{ \pm}\right]=\tau^{ \pm}(b / 2)+\tau^{ \pm}(-b / 2) .
$$

The expression eq. (3.13) is almost the final answer, but we still have to deal with infrared problems. It is immediate to see that there are two possible sources of infrared singularities, which should be removed by the normalisation constant $\mathcal{Z}$. The first one comes from the area of the rectangles, and can be removed by including in $\mathcal{Z}$ the quantity

$$
\mathcal{Z}_{1}=\left\langle\mathcal{W}_{1}\right\rangle\left\langle\mathcal{W}_{2}\right\rangle
$$

where $\mathcal{W}_{1,2}$ are the rectangular Wilson loops describing the free propagation of the mesons (see figure 3). Such a term plays the role of renormalisation constant for the dipoles in a LSZ approach to dipole-dipole scattering [18], and in the gauge/gravity duality approach it is given by

$$
\mathcal{Z}_{1} \simeq e^{-\frac{1}{2 \pi \alpha_{\text {eff }}^{\prime}} A_{\text {rect }}^{\prime}}
$$

where $A_{\text {rect }}^{\prime}$ is the sum of the areas of the minimal surfaces corresponding to the Wilson loops $\mathcal{W}_{1,2}$. For almost vertical walls, the difference $A_{\text {wall }}+A_{\text {rect }}-A_{\text {rect }}^{\prime}$ is approximately of the form

$$
\begin{aligned}
\frac{1}{2 \pi \alpha_{\text {eff }}^{\prime}}\left(A_{\text {wall }}+A_{\text {rect }}-A_{\text {rect }}^{\prime}\right) \simeq & \delta m\left(L^{\text {hel }}\left[\tau^{-}\right]+L^{\text {hel }}\left[\tau^{+}\right]-L_{0}\left[\tau^{-}\right]-L_{0}\left[\tau^{+}\right]\right) \\
& +\delta c\left(L_{0}\left[\tau^{-}\right]+L_{0}\left[\tau^{+}\right]\right),
\end{aligned}
$$

and so it is independent of $T$. The UV divergencies coming from the part of the surface near the boundary $z=0$ are contained in $\delta m$ which, as we will see in a moment, amounts simply to a renormalisation of the (bare) mass parameter $m_{0}$. The second term originates from the incomplete cancellation between the area of the rectangles in the central region (i.e., the region between dashed and dotted lines in figure 3). The quantity $\delta c$ is a UVfinite quantity, which at the present stage we are not able to compute explicitly. However, this term would not affect the variational problem: indeed, it depends on the light-quark trajectories only through $L_{0}\left[\tau^{ \pm}\right]$, which, as we will see in the next subsection, does not enter the minimisation procedure. Therefore, the contribution of this term to the effective action (in the saddle-point approximation considered in this paper) could be determined if $\delta c$ were known. For this reason, we will discuss the possible role of this term in footnotes 21,26 and 32 , dropping it from the main derivation.

The second source of IR singularities is the length of the straight-line part of the lightquark trajectories, which for the dominant paths is expected to be of order $4 T+\mathcal{O}(1)$ for large $T$, so that after analytic continuation $T \rightarrow i T$ we would get an infinite phase $\sim e^{i 4 m_{0} T}$. This phase corresponds to the self-interaction of the quarks, which plays no role in the scattering process, and has therefore to be removed. We then insert a second factor

$$
\mathcal{Z}_{2}=e^{-4 m_{0} T}
$$


which accomplishes this task already at the Euclidean level. The normalisation constant is then taken to be $\mathcal{Z}=\mathcal{Z}_{1} \mathcal{Z}_{2}$. All in all, we obtain for the Euclidean amplitude

$$
\begin{aligned}
\tilde{a}(\vec{b}, \theta, T) \sim \mathcal{N} \Omega^{(f)}\{ & \int \mathcal{D} \tau^{+} \mathcal{D} \tau^{-} \mathcal{F}\left[\tau^{+}, \tau^{-}\right] e^{-\frac{1}{2 \pi \alpha_{\text {eff }}^{\prime}} A_{\min }^{\text {hel }}\left[\tau^{+}, \tau^{-}\right]} \\
& \left.\times e^{-m\left\{L^{\text {hel }}\left[\tau^{-}\right]+L^{\text {hel }}\left[\tau^{+}\right]-L_{0}\left[\tau^{-}\right]-L_{0}\left[\tau^{+}\right]\right\}} \mathcal{U}\left[\tau^{+}, \tau^{-}\right]\right\} \Omega^{(i)},
\end{aligned}
$$

where we have reabsorbed the contribution of the first term in eq. (3.19) in a renormalisation of $m_{0}$, namely $m \equiv m_{0}+\delta m$. As we show in appendix B, the bispinors are eigenvectors with eigenvalue 1 of the (analytic continuation of the) projectors $P\left(\dot{X}_{i}\left(\nu_{i, f}\right)\right)$ acting on them, and so we can replace $\Omega^{(i, f)}$ in eq. (3.21) with the identity. On the other hand, the phase factors $e^{ \pm \frac{i}{2} \Phi\left(\mathcal{C}_{\vec{u}_{i}}\right)}$ contained in $\mathcal{U}$ (see eq. (3.12)) do not cancel, and their effect has to be properly taken into account.

\subsection{Saddle-point approximation}

As anticipated, the final step is a saddle-point approximation of (3.21): exploiting the symmetry of the configuration in order to restrict to the case $\tau^{+}(\sigma)=\tau^{-}(\sigma) \equiv \tau(\sigma)$, one has to solve the Euler-Lagrange equations $\delta S_{\text {eff, } \mathrm{E}}\left[\tau_{\text {s.p. }}(\sigma)\right]=0$, to find the profile $\tau_{\text {s.p. }}(\sigma)$ which minimises the "effective action",

$$
S_{\mathrm{eff}, \mathrm{E}}[\tau(\sigma)] \equiv \frac{1}{2 \pi \alpha_{\mathrm{eff}}^{\prime}} A_{\mathrm{min}}^{\mathrm{hel}}[\tau(\sigma)]+2 m\left(L^{\mathrm{hel}}[\tau(\sigma)]-L_{0}[\tau(\sigma)]\right)
$$

where, with a slight abuse of notation, we have avoided the repetition of the argument in the area functional. In the general case, the variational problem defined in eq. (3.22) is aimed at the determination of an "optimal" boundary, involving in the minimisation procedure both the area of the resulting surface and the length of the boundary. This is what we have called "minimal surface problem with floating boundaries" in the Introduction. ${ }^{14}$

Substituting the solution $\tau_{\text {s.p. }}(\sigma)$ in eq. (3.21) we obtain for the Euclidean amplitude

$$
\tilde{a}(\vec{b}, \theta, T) \sim \mathcal{N} \mathcal{U}\left[\tau_{\text {s.p. }}(\sigma)\right] \mathcal{F}\left[\tau_{\text {s.p. }}(\sigma)\right] e^{-\frac{1}{2 \pi \alpha_{\text {eff }}^{\prime}} A_{\min }^{\text {hel }}\left[\tau_{\text {s.p. }}(\sigma)\right]} e^{-2 m\left\{L^{\text {hel }}\left[\tau_{\text {s.p. }}(\sigma)\right]-L_{0}\left[\tau_{\text {s.p. }}(\sigma)\right]\right\}}
$$

where we have avoided the repetition of the argument also in the fluctuation functional and in $\mathcal{U}$, and we have dropped the $\Omega^{(i, f)}$. The Reggeon trajectory is now encoded in the solution $\tau_{\text {s.p. }}$ of the saddle-point equation: a detailed study of this equation is the subject of the rest of this paper. Since there is no possibility of confusion, in the following we will drop the subscript "s.p." to keep the notation simpler.

A few remarks are now in order. In [14] it was considered explicitly only the case of massless light-fermions. It is immediate to see that in this case a real solution of the EulerLagrange equations does not exist, and the minimal value of $S_{\text {eff, E }}$ for real $\tau(\sigma)$ is obtained

\footnotetext{
${ }^{14}$ Although somewhat similar, this problem must not be confused with the "minimal surface problem with partially free boundary", known in the mathematical literature (see e.g. [72]). In that case, part of the boundary is not competely fixed, but only constrained to lie on a given surface, as in our problem; however, only the area of the surface enters the minimisation procedure, and not the length of the boundary.
} 
for $\tau(\sigma) \equiv 0$, corresponding to a strip of vanishing width connecting the central points of the eikonal trajectories, for which $S_{\text {eff, E }}=2 m b$. The reason for this can be easily understood. The area term in the effective action is of "attractive" nature for the boundaries, since it tends to bend inwards the boundaries in order to minimise the area in between them. On the contrary, the length term is of "repulsive" nature, since it tends to minimise the curvature of the boundaries, in order to minimise their lengths. The value of the quark mass sets the rigidity of the boundaries, and if $m=0$ there is nothing preventing the boundaries to collapse to a strip of vanishing width. In order to start with a non trivial real solution, before analytic continuation, we thus cannot ignore the effect of the length term.

This qualitative argument applies also in a more general setting, with the floating boundaries not constrained to lie on a specific surface. In this setting, we expect that when the quark mass is large the minimisation procedure is dominated by the length term, so that the floating boundaries tend to become straight lines, and the helicoid geometry is lost. As a consequence, the approximation considered here is expected to be valid only for small quark mass.

The solution of the saddle-point equations at $m=0$, found in [14], is indeed a complex solution; more precisely, it is a purely imaginary constant trajectory $\tau(\sigma) \equiv \pm i / p$. Using this solution, and choosing the minus sign for physical reasons, one obtains, after analytic continuation, a Gaussian impact-parameter amplitude, which in turn yields a linear Reggeon trajectory. However, one can immediately check that this solution corresponds to a singular point of the area functional, which reads explicitly

$$
A^{\text {hel }}[\tau(\sigma)]=\frac{1}{p} \int_{-\frac{b}{2}}^{\frac{b}{2}} d \sigma\left[p \tau(\sigma) \sqrt{1+(p \tau(\sigma))^{2}}+\operatorname{arcsinh} p \tau(\sigma)\right],
$$

so that the applicability of the saddle-point method is not guaranteed. In order to investigate this problem more rigorously, it is convenient to start from the case $m \neq 0$, where regular real solutions can be found in Euclidean space: the limit $m \rightarrow 0$ will be considered only after the analytic continuation into Minkowski space-time has been performed.

In the next sections we will study in details the variational problem at hand, which involves the minimisation of a functional which contains both an area and a length term. In particular, in the next section we will study a simpler case, where we can determine exactly and explicitly the solution, in order to obtain a few insights on this kind of problem. The case relevant to Reggeon exchange is discussed in section 5, where we provide an exact solution in implicit form for the general case, and an approximate solution in explicit form in two specific regimes.

\section{Warm-up exercise: soap film with floating boundaries}

Before attacking the minimisation problem relevant to Reggeon exchange in full generality, we want to discuss a simpler case, namely the case in which the straight lines forming the fixed part of the boundary are parallel, i.e., $\theta=0$. This configuration is of limited interest for our problem, since our purpose is to obtain an analytic dependence on $\theta$; nevertheless, the mathematical problem is similar, and moreover in this case the variational equations 


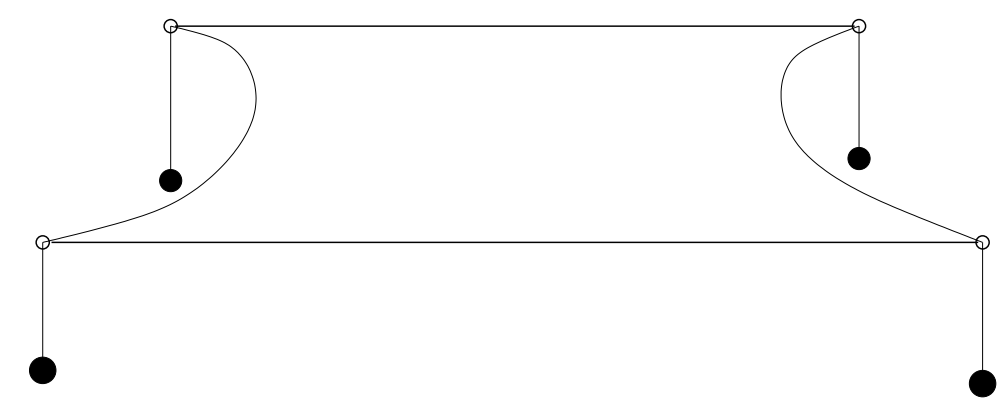

Figure 4. Soap film with partially floating boundary. The straight lines correspond to rigid rods, the curved lines to flexible wires, attached to four equal masses (black balls).

can be solved explicitly, so that we can obtain a few indications in the study of the more complicated "tilted" case $\theta \neq 0$. We consider then the minimisation of the functional

$$
H=\frac{1}{2 \pi \hat{\alpha}^{\prime}} A\left[\mathcal{C}_{1}, \mathcal{C}_{2}\right]+\hat{m}\left(L\left[\mathcal{C}_{1}\right]+L\left[\mathcal{C}_{2}\right]\right)
$$

where $A$ is the area of a surface bounded on two opposite sides by two parallel straight lines of length $2 T$ at a distance $R$, which are held fixed. On the other sides, the surface is bounded by two a priori free lines following the paths $\mathcal{C}_{1,2}$, of length $L\left[\mathcal{C}_{1,2}\right]$, which have to be determined by the minimisation procedure.

For want of a physical interpretation, this functional corresponds to the energy of an ideal soap film of vanishing mass and of surface tension $1 / 2 \pi \hat{\alpha}^{\prime}$, extending between two rigid rods (the straight lines) parallel to the ground, and between two flexible (massless) wires (of length larger than $2 T$ ), each passing through two rings positioned at the endpoints of the rods (see figure 4); moreover, two equal masses $M$ are attached at the endpoints of each wire, with $M g=\hat{m}$, and their potential energy in the gravitational field contributes the length term.

Given the symmetries of the problem, the solution will be a planar surface, and the two floating boundaries will be one the reflection of the other. The problem is thus effectively two-dimensional, and we can parameterise the relevant surfaces in terms of a single function $\tau(\sigma)$, i.e.,

$$
X^{\text {plan }}[\tau(\sigma) ; \bar{\tau}, \sigma]=(\bar{\tau}, \sigma), \quad \sigma \in[-R / 2, R / 2], \quad \bar{\tau} \in[-\tau(\sigma), \tau(\sigma)], \tau(\sigma)>0 .
$$

The expression of the functionals simplifies therefore to

$$
L=\int_{-\frac{R}{2}}^{+\frac{R}{2}} d \sigma \sqrt{1+(\dot{\tau}(\sigma))^{2}}, \quad A=2 \int_{-\frac{R}{2}}^{+\frac{R}{2}} d \sigma \tau(\sigma) .
$$

Notice that $\tau$ must satisfy $\tau(\sigma)=\tau(-\sigma)$ because of the symmetries of the problem. The Euler-Lagrange equation is easily derived, and reads

$$
\ddot{\tau}-2 R_{c}^{-1}\left(1+\dot{\tau}^{2}\right)^{\frac{3}{2}}=0,
$$

where the combined parameter

$$
R_{c} \equiv 4 \pi \hat{\alpha}^{\prime} \hat{m}
$$


will play an important role as a critical value for $R$ in the minimisation problem. Notice that for $R_{c}>0$ we have $\ddot{\tau}>0$. This equation reflects the general expectation on the nature of the two terms contributing to the energy functional, discussed in the previous section. For large $R_{c}$ the first "length" term in (4.4) dominates, so that the equation reduces to that of a straight line; the second "area" term increases the curvature of the free boundary, bending it inwards. This equation is solved in the standard way by setting ${ }^{15}$

$$
v(\tau)=\sqrt{1+\dot{\tau}^{2}}, \quad v^{\prime} \equiv \frac{d v}{d \tau}=(\dot{\tau})^{-1} \frac{d v}{d \sigma}=\frac{\ddot{\tau}}{v} .
$$

The equation becomes then

$$
v^{\prime}=2 R_{c}^{-1} v^{2}
$$

which is easily solved by

$$
v(\tau)=\frac{v\left(\tau_{0}\right)}{1-2 R_{c}^{-1} v\left(\tau_{0}\right)\left(\tau-\tau_{0}\right)}=\frac{1}{1-2 R_{c}^{-1}\left(\tau-\tau_{0}\right)},
$$

where $\tau_{0}=\tau(0)$, and we have taken into account that $v\left(\tau_{0}\right)=\sqrt{1+(\dot{\tau}(0))^{2}}=1$, since $\dot{\tau}(0)=0$. Notice that, since $1 \leq v \leq \infty$, we have to satisfy $0 \leq\left(\tau-\tau_{0}\right) \leq R_{c} / 2$. Proceeding in the usual way, we write

$$
|\sigma|=\int_{\tau_{0}}^{\tau(\sigma)} \frac{d \tau}{\sqrt{v^{2}-1}}=\sqrt{\left(\tau(\sigma)-\tau_{0}\right)\left[R_{c}-\left(\tau(\sigma)-\tau_{0}\right)\right]},
$$

which can be inverted to give

$$
\tau(\sigma)-\tau_{0}=\frac{R_{c}}{2}\left[1-\sqrt{1-\left(\frac{2 \sigma}{R_{c}}\right)^{2}}\right],
$$

where the minus sign for the square root has been chosen since the left-hand side of the equation vanishes at $\sigma=0$. Finally, imposing the boundary condition $\tau(R / 2)=T$, we obtain the complete solution ${ }^{16}$ (see figure 5)

$$
\tau_{\min }(\sigma)=T-\frac{R_{c}}{2}\left[\sqrt{1-\left(\frac{2 \sigma}{R_{c}}\right)^{2}}-\sqrt{1-\left(\frac{R}{R_{c}}\right)^{2}}\right] .
$$

Notice that in order for $\tau(\sigma)$ to be real we need the following condition to be satisfied:

$$
R \leq R_{c}
$$

This justifies the notation chosen for $R_{c} \equiv 4 \pi \hat{\alpha}^{\prime} \hat{m}$. The geometric meaning of this condition

\footnotetext{
${ }^{15}$ The equation could have been solved by direct integration, but the present approach generalises immediately to non-constant $R_{c}$, which will be relevant in the next section.

${ }^{16}$ As we will discuss in detail in the next section, this is not the boundary condition that we impose in the $\theta \neq 0$ case, so this result does not give the $\theta \rightarrow 0$ limit of the calculation relevant to Reggeon exchange.
} 


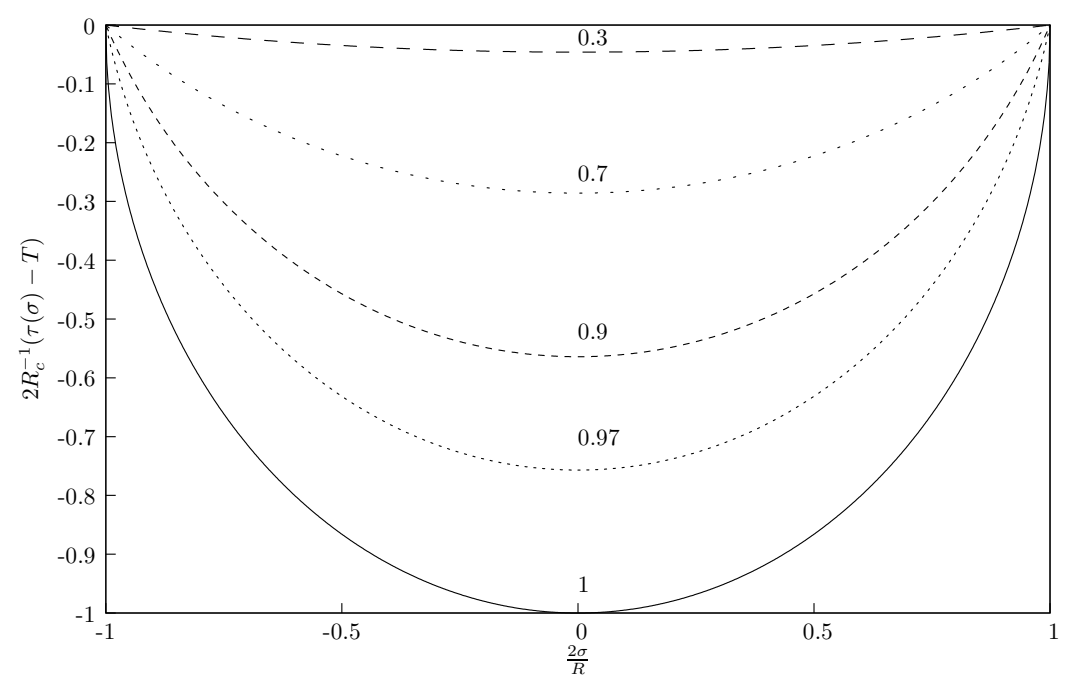

Figure 5. Minimisation profile of the floating boundary. Half of the floating boundary is represented after minimisation for various values of $R / R_{c}$. The opposite half of the floating boundary is obtained by reflection with respect to the horizontal axis.

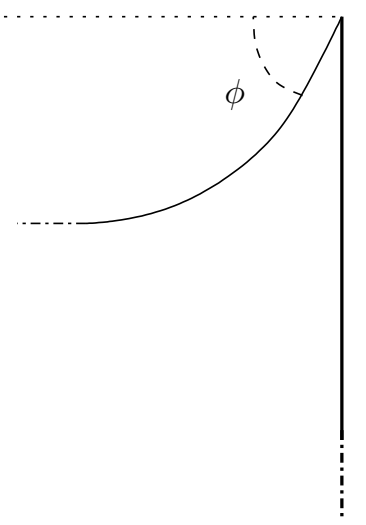

Figure 6. The angle $\phi$ formed by the fixed and the floating boundaries at the soap film corner. The angle is given by the tangent of $\tau(\sigma)$ at $\sigma=R / 2$, cfr. eq. (4.13).

is clarified by computing the derivative of $\tau(\sigma)$ at $\sigma=R / 2$,

$$
\left.\dot{\tau}(\sigma)\right|_{\sigma=R / 2}=\frac{R / R_{c}}{\sqrt{1-\left(R / R_{c}\right)^{2}}}=\tan \phi,
$$

where the angle $\phi$ is shown in figure 6 . It is then immediate to see that the condition eq. (4.12) simply means that $\phi \leq \pi / 2$, and when the bound is reached the flexible wire runs parallel to the rigid rod at the junction point. The physical interpretation of this condition will be discussed in a moment.

We turn now to the computation of the energy corresponding to the solution $\tau_{\min }$. Making use of the properties of the minimal solution, it can be expressed in a simple form, 
which is readily evaluated:

$$
\begin{aligned}
H_{\min } & =\frac{2}{\pi \hat{\alpha}^{\prime}} \int_{0}^{\frac{R}{2}} d \sigma\left\{\tau_{0}+\frac{R_{c}}{2}\left(1+\frac{\left(2 R_{c}^{-1} \sigma\right)^{2}}{\sqrt{1-\left(2 R_{c}^{-1} \sigma\right)^{2}}}\right)\right\}= \\
& =\frac{1}{2 \pi \hat{\alpha}^{\prime}}\left\{2 T R+\frac{R R_{c}}{2} \sqrt{1-\left(\frac{R}{R_{c}}\right)^{2}}+\frac{R_{c}^{2}}{2} \arcsin \frac{R}{R_{c}}\right\} .
\end{aligned}
$$

In the limit $R_{c} \rightarrow \infty$, the weight of the masses attached to the wires wins over the surface tension, so that the wires are kept straight, and we recover the result for a soap film with a fixed rectangular boundary, i.e., $\tau_{\min } \rightarrow T$, and

$$
H_{\min } \simeq \frac{1}{2 \pi \hat{\alpha}^{\prime}}\left\{2 T R+R R_{c}\right\} .
$$

At fixed $R$ we cannot go to the limit $R_{c} \rightarrow 0$, since in this case eq. (4.12) implies that $R_{c}$ must be bounded from below. The meaning of eq. (4.12) is the following: if $R$ exceeds the critical value $R_{c}$ at fixed $\hat{\alpha}$ and $\hat{m}$ (more precisely, at fixed $\hat{\alpha} \hat{m}$ ), or equivalently if $R_{c}$ becomes smaller than $R$ (e.g. for too large surface tension or too small mass), the force due to the surface tension is stronger than the gravitational force on the masses, and it makes the soap film collapse. This is essentially a Gross-Ooguri transition [63], which we expect to find also in the case $\theta \neq 0$, and $R_{c}$ appears to be the corresponding critical value at which the transition takes place. Notice that rewriting the energy in terms of the angle $\phi$ as

$$
H_{\min }=\hat{m}\left\{4 T \sin \phi+R_{c}(\phi+\sin \phi \cos \phi)\right\},
$$

we easily see that the maximal value is reached for $\phi=\pi / 2$, i.e., right before the collapse, so that the maximal energy that can be stored in this system is $\hat{m}\left\{4 T+\pi / 2 R_{c}\right\}$.

As a final remark, we note in passing that identifying $\frac{1}{2 \pi \hat{\alpha}^{\prime}}$ with the string tension $\sigma$ and $\hat{m}$ with the constituent quark mass $m$ (the functional $H$ having now the dimensions of an action), the minimisation procedure reproduces the static $Q-\bar{Q}$ linear potential $V_{Q \bar{Q}}(R)=\sigma R$ as $V_{Q \bar{Q}}(R)=H_{\min } / 2 T$ for $T \rightarrow \infty$, and moreover the bound $R \leq R_{c}$ gives the well-known string-breaking condition $\sigma R \leq 2 m$.

\section{Variational problem for the Reggeon-exchange amplitude in Euclidean space}

In this section we discuss the Euclidean variational problem relevant to the Reggeonexchange amplitude, i.e., for the "tilted" configuration of figure 2. As we have already said in section 3, the scattering amplitude is reconstructed through analytic continuation from the solution of the minimal surface problem with floating boundaries, that involves the "effective action" functional eq. (3.22), which we rewrite here for convenience:

$$
S_{\text {eff, } \mathrm{E}}[\tau(\sigma)]=\frac{1}{2 \pi \alpha_{\text {eff }}^{\prime}} A_{\text {min }}^{\text {hel }}[\tau(\sigma)]+2 m\left(L^{\text {hel }}[\tau(\sigma)]-L_{0}[\tau(\sigma)]\right) .
$$


Our aim is to find a smooth "profile" $\tau(\sigma)$, bounding a piece of helicoid which connects two straight lines at a transverse distance $b$, and forming an angle $\theta$ in the longitudinal $\left(x_{4}-x_{1}\right)$ plane. In order to do so, it is convenient to pass to dimensionless coordinates by making the change of variables

$$
t(s)=p \tau(\sigma), \quad s=p \sigma, \quad p x=y, \quad \text { with } \quad p=\theta / b .
$$

Note that $\dot{t} \equiv \frac{d t}{d s}=\frac{d \tau}{d \sigma}=\dot{\tau}$. In terms of these reduced variables, the expressions for the area and length functionals eq. (3.14) read

$$
\begin{aligned}
A_{\min }^{\mathrm{hel}} & =\frac{1}{p^{2}} \int_{-\frac{\theta}{2}}^{+\frac{\theta}{2}} d s \int_{-t(s)}^{+t(s)} d y \sqrt{1+y^{2}}, \\
L^{\mathrm{hel}} & =\frac{1}{p} \int_{-\frac{\theta}{2}}^{+\frac{\theta}{2}} d s \sqrt{1+[t(s)]^{2}+[\dot{t}(s)]^{2}},
\end{aligned}
$$

and moreover

$$
L_{0}=\frac{1}{p}\left[t\left(\frac{\theta}{2}\right)+t\left(-\frac{\theta}{2}\right)\right],
$$

for the subtraction term. This term will not enter the variational equations, since the value of $\tau\left( \pm \frac{b}{2}\right)$, and so that of $t\left( \pm \frac{\theta}{2}\right)$, is determined by requiring a smooth transition to the eikonal straight-line paths: in other words, we perform the variation of the effective action at $t\left( \pm \frac{\theta}{2}\right)$ fixed, we solve the equation and we subsequently determine the value which makes the path smooth. In terms of our parameterisation, in order for the part of the path on the helicoid to be smoothly connected with the incoming and outgoing straight lines, we need that $\dot{t}\left( \pm \frac{\theta}{2}\right)= \pm \infty$. We will discuss this point in more detail after solving the general equation.

\subsection{Exact solution in implicit form}

It is straightforward to obtain the Euler-Lagrange equation corresponding to the minimisation of the functional, which reads explicitly

$$
\frac{2 m}{p} \frac{1}{\left(1+t^{2}+\dot{t}^{2}\right)^{\frac{3}{2}}}\left[(\ddot{t}-t)\left(1+t^{2}\right)-2 t \dot{t}^{2}\right]-\frac{1}{\pi \alpha_{\text {eff }}^{\prime} p^{2}} \sqrt{1+t^{2}}=0 .
$$

After setting

$$
t(s)=\frac{\theta}{b} \tau(\sigma) \equiv \sinh \varphi(s), \quad \lambda \equiv \frac{1}{2 \pi \alpha_{\mathrm{eff}}^{\prime} m p}=\frac{b}{2 \pi \alpha_{\mathrm{eff}}^{\prime} m \theta},
$$

the equation takes the simpler form

$$
\ddot{\varphi}-\left(1+\dot{\varphi}^{2}\right) \tanh \varphi-\lambda\left(1+\dot{\varphi}^{2}\right)^{\frac{3}{2}} \cosh \varphi=0 .
$$

In some loose sense, the variable $\varphi$ parameterises in a scale-invariant way the development in "time" of the quark-exchange process in Euclidean space.

As shown in the previous section, in order to solve this equation one sets

$$
v(\varphi)=\sqrt{1+\dot{\varphi}^{2}} \longrightarrow v v^{\prime}=\ddot{\varphi},
$$


where the prime denotes differentiation with respect to $\varphi$. The equation becomes then

$$
v^{\prime}-v \tanh \varphi-\lambda v^{2} \cosh \varphi=0,
$$

which has the general solution

$$
v(\varphi)=\frac{v_{0} \cosh \varphi}{\cosh \varphi_{0}+v_{0} \frac{\lambda}{2}\left(f\left(\varphi_{0}\right)-f(\varphi)\right)}, \quad v_{0}=v\left(\varphi_{0}\right),
$$

where the function

$$
f(x) \equiv x+\sinh x \cosh x
$$

plays an important role in the following.

The symmetries of the problem imply $\varphi(-s)=\varphi(s),{ }^{17}$ and as a consequence $\dot{\varphi}(0)=0$; in turn, we have $v\left(\varphi_{0}\right)=1$. Notice that since $\ddot{\varphi}>0$ (unless $\lambda=0$, in which case the area term is absent) we have $\varphi(s)>\varphi_{0}$ for $s \neq 0$, as one expects for a minimum. Moreover, the positivity of $v$ and the monotonicity of $f(x)$ imply that $\varphi(s)$ must satisfy $0 \leq f(\varphi)-f\left(\varphi_{0}\right) \leq(2 / \lambda) \cosh \varphi_{0}$.

It is immediate at this point to write down the general solution of our variational equation, which reads

$$
|s|=\int_{\varphi_{0}}^{\varphi(s)} d x \frac{1}{\sqrt{v(x)^{2}-1}} .
$$

To fully determine the solution of the variational problem we still have to impose the appropriate boundary conditions. In order to do so, it is convenient to define $\tilde{\varphi}$ through the equation

$$
\cosh \varphi_{0}+\frac{\lambda}{2}\left(f\left(\varphi_{0}\right)-f(\tilde{\varphi})\right)=0
$$

and so rewrite $v$ as

$$
v(\varphi)=\frac{\cosh \varphi}{\frac{\lambda}{2}(f(\tilde{\varphi})-f(\varphi))} .
$$

The value $\tilde{\varphi}$ is the maximal allowed value for $\varphi(s)$ which respects the positivity of $v$, i.e., whatever is the boundary condition that we choose, we still need the inequality $\varphi_{0} \leq$ $\varphi(s) \leq \tilde{\varphi}$ to be satisfied. Since we look for a path on the helicoid that at $\sigma= \pm b / 2$, or equivalently at $s= \pm \theta / 2$, joins smoothly the eikonal incoming and outgoing trajectories of the exchanged light fermions, the derivative $\dot{t}=\dot{\varphi} \cosh \varphi$ has to diverge at $s= \pm \theta / 2$. As the function $v$, and thus $\dot{\varphi}$, have a divergence at the point $\tilde{\varphi}$, the appropriate boundary condition is then precisely $\varphi\left( \pm \frac{\theta}{2}\right)=\tilde{\varphi} .^{18}$ We will comment further on this point at the end of this section. Using eq. (5.12), this boundary condition is expressed as

$$
\frac{\theta}{2}=\int_{\varphi_{0}}^{\tilde{\varphi}} d \varphi \frac{1}{\sqrt{v(\varphi)^{2}-1}}=\int_{\varphi_{0}}^{\tilde{\varphi}} d \varphi \frac{\frac{\lambda}{2}(f(\tilde{\varphi})-f(\varphi))}{\sqrt{\cosh ^{2} \varphi-\left[\frac{\lambda}{2}(f(\tilde{\varphi})-f(\varphi))\right]^{2}}}
$$

\footnotetext{
${ }^{17}$ Strictly speaking, this is true only if the solution is unique. Since we are dealing with a boundary value problem for a nonlinear differential equation, as we will explain shortly, we are not guaranteed a priori of the existence and unicity of the solution. Nevertheless, we have verified that the solution that we have found is actually unique.

${ }^{18}$ The other possibility would be $\varphi\left( \pm \frac{\theta}{2}\right)=\infty$, but since $\varphi(s) \leq \tilde{\varphi}$ this would again require $\infty=\varphi\left( \pm \frac{\theta}{2}\right) \leq$ $\tilde{\varphi}=\infty$.
} 


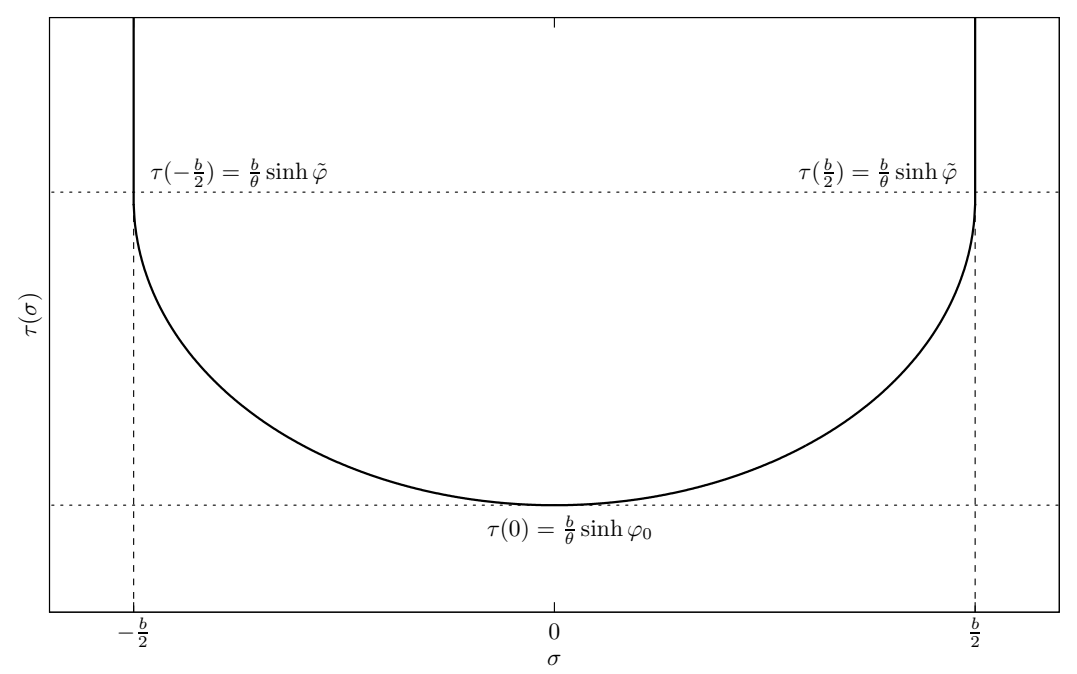

Figure 7. Sketch of the minimisation profile $\tau(\sigma)$ described by the trajectories of the exchanged fermions on the helicoid. The solid line represents the trajectory of the exchanged fermion. The dashed (vertical) lines are the eikonal trajectories, plotted for reference. The dotted (horizontal) lines indicate the minimal and maximal values of $\tau(\sigma)$, i.e., $\tau(0)=\frac{b}{\theta} \sinh \varphi_{0}$ and $\tau\left( \pm \frac{b}{2}\right)=\frac{b}{\theta} \sinh \tilde{\varphi}$.

and the mathematical problem is now completely specified. Equations (5.13) and (5.15) form a coupled set of equations, whose solution would give the explicit form of the profile $\tau(\sigma)$. We have not yet been able to find an analytic solution in the general case. Some approximate solutions will be discussed in the next subsection; here we discuss some general properties of the result. A qualitative sketch of the solution is shown in figure 7 .

As a first observation, we notice that the values $\varphi_{0}$ and $\tilde{\varphi}$ are related to the characteristic time scales of the quark-exchange interaction in Euclidean space. Recall that in the dual string picture the interaction is described by the exchange in the $t$-channel of an open string with helicoidal world-sheet between the colliding mesons. The world-sheet coordinates are $\sigma \in[-b / 2, b / 2]$ and $\bar{\tau} \in[-\tau(\sigma), \tau(\sigma)]$ (see eq. (3.10)), and they are related to the Euclidean time $t_{E}=x_{4}$ by the relation $t_{E}=\bar{\tau} \cos (\theta \sigma / b)$. The development of the interaction, seen as a process taking place in Euclidean time, passes through the following three stages. In the first stage, at time $t_{E i}=-\tilde{\tau} \cos (\theta / 2)$, with $\tilde{\tau}=(b / \theta) \sinh \tilde{\varphi}$, the strings corresponding to the two scattering mesons in the initial state begin to expand; the expansion continues until $t_{E-}=-\tilde{\tau}_{0}$, where $\tilde{\tau}_{0}=(b / \theta) \sinh \varphi_{0}$, when they join forming a single, unstable string. The second stage corresponds to the existence of this unstable string, which lasts until $t_{E+}=\tilde{\tau}_{0}$, when it decays and splits in two. In the third stage, the decay products shrink, returning to their initial size at $t_{E f}=\tilde{\tau} \cos (\theta / 2)$, thus reconstituting the mesons in the final state.

An important remark is that the solution does not depend on the length variable $T:{ }^{19}$ this guarantees that our result will be free of IR divergencies. This is actually expected,

\footnotetext{
${ }^{19}$ Strictly speaking, a solution exists only if $T \geq \tau( \pm b / 2)$, but since we are interested in the limit $T \rightarrow \infty$ this restriction is irrelevant.
} 
since we are working with colourless objects, and it is in contrast with the divergencies arising in quark-quark and gluon-gluon scattering $[12,58]$. The only way in which $T$ could have entered the solution is through the boundary conditions, since the equations do not depend on it, but our choice for them is again independent of $T$. As a consequence, the relevant analytic continuation from Euclidean to Minkowski space-time reduces simply to $\theta \rightarrow-i \chi$.

It is easy to prove that a regular solution, for which $\tau(s)>0$, and thus $\varphi(s)>0$, can exist only in a limited range for the impact parameter. From eq. (5.7), using the fact that $\varphi(s) \geq \varphi_{0}$, we derive the inequality

$$
\frac{\ddot{\varphi}}{\left(1+\dot{\varphi}^{2}\right)^{\frac{3}{2}}} \geq \lambda \cosh \varphi \geq \lambda \cosh \varphi_{0}
$$

which, integrating between 0 and $\theta / 2$, and using $\dot{\varphi}(0)=0$ and $\dot{\varphi}(\theta / 2)=\infty$, provides a bound on $b$,

$$
\frac{\lambda \theta}{2}=\frac{b}{4 \pi \alpha_{\mathrm{eff}}^{\prime} m} \leq \frac{1}{\cosh \varphi_{0}} \leq 1
$$

This defines a critical value

$$
b_{c} \equiv 4 \pi \alpha_{\mathrm{eff}}^{\prime} m,
$$

beyond which the Euclidean solution ceases to be a positive real quantity. The limitation imposed by this bound is analogous to the one found in the case $\theta=0$, eq. (4.12), i.e., for too large $b$ the four-dimensional Euclidean "soap film" corresponding to the string worldsheet collapses due to the attractive effect of the string tension. ${ }^{20}$ Moreover, the fact that $b_{c}$ vanishes when $m=0$ reflects the necessity of a "repulsive" boundary-length term to compensate for the "attractive" area term in the minimisation procedure.

Another inequality can be obtained by multiplying by $\dot{\varphi}$ both sides of the first relation in eq. (5.16), and integrating between 0 and $\theta / 2$,

$$
1 \geq \lambda\left(\sinh \tilde{\varphi}-\sinh \varphi_{0}\right)=\frac{1}{2 \pi \alpha_{\mathrm{eff}}^{\prime} m}\left(\tau\left(\frac{b}{2}\right)-\tau(0)\right) .
$$

This result implies that the variation of $\tau$ along the boundary of the helicoid is of order $\mathcal{O}\left(\alpha_{\text {eff }}^{\prime} m\right)$ : as a consequence, in the massless case the profile which minimises the effective action should be a constant. However, no constant real solution of eq. (5.7) exists; moreover, even if it existed, it could not be smooth at the junction with the eikonal incoming and outgoing trajectories. It is simple to derive from (5.19) an inequality for $\Delta \equiv \tilde{\varphi}-\varphi_{0}$,

$$
1 \geq \lambda \cosh \varphi_{0} \sinh \Delta \geq \lambda \Delta,
$$

which will be useful in the next subsection.

\footnotetext{
${ }^{20}$ The interpretation of eq. (5.17) as a string-breaking condition suggests that the mass parameter $m$ represents the constituent mass of the light quark. Together with the lower bound on $b$ discussed previously, this condition determines a window $R_{0} \lesssim b \leq b_{c}$, where the flat-space approximation is expected to be valid.
} 
We can now exploit the formal solution, and the relations eq. (5.13) and (5.15) satisfied by $\varphi_{0}$ and $\tilde{\varphi}$, in order to rewrite the effective-action functional in a rather compact way, namely $^{21}$

$$
S_{\mathrm{eff}, \mathrm{E}}=\frac{b^{2}}{2 \pi \alpha_{\mathrm{eff}}^{\prime} \theta} f(\tilde{\varphi})+\frac{4 m b}{\theta}\left(B\left(\varphi_{0}, \tilde{\varphi}\right)-\sinh \tilde{\varphi}\right)
$$

where

$$
B\left(\varphi_{0}, \tilde{\varphi}\right)=\int_{\varphi_{0}}^{\tilde{\varphi}} d \varphi \sqrt{(\cosh \varphi)^{2}-\left[\frac{\lambda}{2}(f(\tilde{\varphi})-f(\varphi))\right]^{2}},
$$

and we recall that

$$
f(\varphi)=\varphi+\sinh \varphi \cosh \varphi .
$$

The last term in eq. (5.21) is simply the subtraction term $2 m L_{0}$, rewritten in terms of $\tilde{\varphi}$. The other two terms are obtained by combining the expressions for the area of the piece of helicoid and the length of its curved boundaries. Since the area of a portion of helicoid with a constant profile $\tau(\sigma) \equiv \bar{\tau}$ can be expressed as $A(\theta, b, \bar{\tau})=\left(b^{2} / \theta\right) f(\operatorname{arcsinh}(\theta \bar{\tau} / b))$, one recognises in the first term the area $\bar{A}$ of such a surface, with $\bar{\tau}=(b / \theta) \sinh \tilde{\varphi}$, times a factor $1 / 2 \pi \alpha_{\text {eff }}^{\prime}$. Moreover, it is easily proved that $B\left(\varphi_{0}, \tilde{\varphi}\right)-\sinh \tilde{\varphi} \leq 0$, so that the effective action is actually smaller than $\bar{A} / 2 \pi \alpha_{\text {eff }}^{\prime}$.

As anticipated in the Introduction, the minimal effective action eq. (5.21), with the functions $B\left(\varphi_{0}, \tilde{\varphi}\right)$ defined by $(5.22)$ and $f(\tilde{\varphi})$ by $(5.23)$, represents the main result of the variational problem discussed in the present section, and it encodes the properties of Reggeon exchange in a compact analytic form. In order to be of practical use, it requires the explicit solution of the system of equations (5.13) and (5.15). Before moving on to this issue, which is the subject of the next subsection, we want to comment briefly on two points.

Although in this paper we have focussed only on positive real solutions of (5.13) and (5.15), this system of equations admits also solutions for which $\varphi_{0}$ is negative. As long as the equations for the boundary conditions give $\tilde{\varphi} \geq 0$, a profile can be formally defined, which at a certain value $\pm \bar{s}$ vanishes, and is negative for $|s| \leq \bar{s}$. This simply means that the curves corresponding to the propagation of the light fermions cross at a certain point; as a consequence, the profile obtained by replacing the piece between the two crossing points with a straight line would yield a smaller value for the effective action. More precisely, if $\varphi(s)$ is a solution of the minimisation equations which vanishes at $\pm \bar{s}$, one substitutes $\varphi(s) \rightarrow \varphi(s) \theta\left(s^{2}-\bar{s}^{2}\right)$, resulting in a surface contracted to a vanishing strip in the central region. Of course, the value which results for the effective action is no longer given by formula (5.21), which has to be modified taking into account the different shape of the central region. The resulting profile is continuous, but it has cusps at $\pm \bar{s}$, which require a careful evaluation of the spin factor. Finally, when $\tilde{\varphi}=0$, the surface becomes just a thin strip of vanishing width connecting the eikonal trajectories; the cusps are found at $\pm \theta / 2$, and the cusp angle is $\pi / 2$. In the rest of this paper we will not discuss these configurations anymore, focusing only on smooth solutions with $\tau(\sigma) \geq 0$.

\footnotetext{
${ }^{21}$ We note in passing that the term neglected in eq. (3.19) would give to eq. (5.21) the contribution $\delta S_{\text {eff }, \mathrm{E}}=\delta c(4 b / \theta) \sinh \tilde{\varphi}$.
} 
We would also remark that the choice $\varphi\left( \pm \frac{\theta}{2}\right)=\tilde{\varphi}$, dictated by the smoothness condition, corresponds actually to the minimal value of the effective action among the solutions of eq. (5.7). Indeed, one can consider the most general choice $\varphi\left( \pm \frac{\theta}{2}\right)=\bar{\varphi} \leq \tilde{\varphi}$ (larger values are not allowed, see the comment after eq. (5.14)), thus introducing cusps at $s= \pm \frac{\theta}{2}$. This yields for the effective action

$$
S_{\mathrm{eff}, \mathrm{E}}(\bar{\varphi})=\frac{b^{2}}{2 \pi \alpha_{\mathrm{eff}}^{\prime} \theta} f(\tilde{\varphi})+\frac{4 m b}{\theta}\left(\bar{B}\left(\varphi_{0}, \tilde{\varphi}, \bar{\varphi}\right)-\sinh \bar{\varphi}\right)
$$

where

$$
\bar{B}\left(\varphi_{0}, \tilde{\varphi}, \bar{\varphi}\right)=\int_{\varphi_{0}}^{\bar{\varphi}} d \varphi \sqrt{(\cosh \varphi)^{2}-\left[\frac{\lambda}{2}(f(\tilde{\varphi})-f(\varphi))\right]^{2}},
$$

and $\tilde{\varphi}$ is again defined by eq. (5.13). The boundary condition eq. (5.15) becomes

$$
\frac{\theta}{2}=\int_{\varphi_{0}}^{\bar{\varphi}} d \varphi \frac{\frac{\lambda}{2}(f(\tilde{\varphi})-f(\varphi))}{\sqrt{\cosh ^{2} \varphi-\left[\frac{\lambda}{2}(f(\tilde{\varphi})-f(\varphi)]^{2}\right.}} .
$$

Equations (5.13) and (5.26) define implicitly the dependence of $\varphi_{0}$ and $\tilde{\varphi}$ on the boundary value $\bar{\varphi}$. In order to find the value of $\bar{\varphi}$ which minimises the effective action, one has to compute the derivative

$$
\frac{d S_{\mathrm{eff}, \mathrm{E}}}{d \bar{\varphi}}=\frac{d \varphi_{0}}{d \bar{\varphi}} \frac{\partial S_{\mathrm{eff}, \mathrm{E}}}{\partial \varphi_{0}}+\frac{d \tilde{\varphi}}{d \bar{\varphi}} \frac{\partial S_{\mathrm{eff}, \mathrm{E}}}{\partial \tilde{\varphi}}+\frac{\partial S_{\mathrm{eff}, \mathrm{E}}}{\partial \bar{\varphi}}
$$

however, one easily sees that $\frac{\partial S_{\text {eff, E }}}{\partial \varphi_{0}}=0$ upon use of eq. (5.13), and moreover $\frac{\partial S_{\text {eff, E }}}{\partial \tilde{\varphi}}=0$ upon use of eq. (5.26). One is thus left with

$$
\frac{d S_{\mathrm{eff}, \mathrm{E}}}{d \bar{\varphi}}=\frac{\partial S_{\mathrm{eff}, \mathrm{E}}}{\partial \bar{\varphi}}=\frac{4 m b}{\theta}\left(\sqrt{\cosh ^{2} \bar{\varphi}-\left[\frac{\lambda}{2}(f(\tilde{\varphi})-f(\bar{\varphi}))\right]^{2}}-\cosh \bar{\varphi}\right)
$$

and so $\frac{d S_{\text {eff, }}}{d \bar{\varphi}}<0$ for $\bar{\varphi}<\tilde{\varphi}$. Therefore, $S_{\text {eff, E }}$ is minimal for the maximal allowed value of $\bar{\varphi}$, i.e., $\bar{\varphi}=\tilde{\varphi}$.

\subsection{Explicit solutions: analytical and numerical results}

In order to perform correctly the analytic continuation, one should obtain the exact dependence on $\theta$ by solving the equations eq. (5.13) and (5.15) for $\varphi_{0}$ and $\tilde{\varphi}$, and inserting them in the formula for the effective action. This is a hard problem, which we have not been able to solve in the general case, and so, in order to investigate the analytic dependence on $\theta$, and on the impact parameter $b$, we have to focus on some specific regimes where the relevant expressions simplify, and the equations become manageable.

One possibility is to consider the case of large $\varphi_{0}$ (and therefore also large $\tilde{\varphi}$ ): in this case we can approximate $f(x) \simeq e^{2 x} / 4$ in (5.23), and solve explicitly equations eq. (5.13) and (5.15). From eq. (5.20), we see that this approximation holds in the region where $\lambda \sinh \Delta \ll 1$, which corresponds to small $b$, as we will show. As we will see, this region is of limited physical interest; moreover, it is not possible to calculate higher-order corrections straightforwardly. 
Another possibility is to consider the case of large $\lambda$, which, according to the inequality eq. (5.20), implies $\Delta \ll 1$. Since $b$ is limited, this regime corresponds essentially to small values of $\theta .{ }^{22}$ In this case we can perform an expansion in powers of $\Delta$ of the various quantities, and then solve explicitly the equations. This can be done in a systematic way, but here we focus on the lowest order approximation only, briefly commenting on higherorder corrections. As we will see, this case turns out to be physically relevant after analytic continuation to Minkowski space-time.

In order to obtain an overview of the general features of the solution, and of the corresponding value of the effective action, in a wider range of values of $b$ and $\theta$, we have solved the equations numerically. Although we cannot determine the analytic form of the solution for $\varphi_{0}$ and $\tilde{\varphi}$, and therefore that of the effective action, nevertheless the numerical results can help in understanding better the various regimes of the solution for the minimisation problem, and the range of validity of our approximate analytic expressions. Moreover, the numerical investigation of the solution reveals a few features which are not captured by the available analytic results. Our numerical results are shown in figures 8-10, and compared with the analytic approximations.

\subsubsection{Case $\varphi_{0} \gg 1$}

We consider first the case of large $\varphi_{0}$. As we will see, this corresponds to a region where $b / 4 \pi m \alpha_{\text {eff }}^{\prime}=b / b_{c}$ is small. We begin by solving the equations (5.13) and (5.15) for the boundary values $\varphi_{0}$ and $\tilde{\varphi}$. Retaining only the leading terms in eq. (5.13), i.e., approximating $f(x) \simeq e^{2 x} / 4$ and $\cosh x \simeq e^{x} / 2$, we find

$$
1 \simeq \frac{\lambda}{2} e^{\tilde{\varphi}} \sinh \Delta .
$$

It is easy to see that if this equation has a solution with large $\tilde{\varphi}$, then $\lambda \sinh \Delta$ must be small, as expected from (5.20). Making the same approximation in eq. (5.15) we obtain

$$
\frac{\theta}{2} \simeq \int_{0}^{\Delta} d x \frac{\sinh x}{\sqrt{\sinh ^{2} \Delta-\sinh ^{2} x}}=\arcsin \tanh \Delta,
$$

where we have used (5.29) and the change of variables

$$
y=\sqrt{1-\left(\frac{\cosh x}{\cosh \Delta}\right)^{2}} .
$$

We can then write down the solution as

$$
\begin{aligned}
& \tilde{\varphi} \simeq \log \left(\frac{b_{c}}{b} \theta \cot \frac{\theta}{2}\right) \\
& \Delta \simeq \operatorname{arctanh} \sin \frac{\theta}{2}=\frac{1}{2} \log \left(\frac{1+\sin \frac{\theta}{2}}{1-\sin \frac{\theta}{2}}\right) .
\end{aligned}
$$

\footnotetext{
${ }^{22}$ Notice that there is a partial overlap with the range of validity of the approximation discussed above.
} 
Explicitly, we have for $\varphi_{0}$

$$
\varphi_{0}=\tilde{\varphi}-\Delta=\log \left(\frac{b_{c}}{b} \theta \cot \frac{\theta}{2} \sqrt{\frac{1-\sin \frac{\theta}{2}}{1+\sin \frac{\theta}{2}}}\right),
$$

and the condition $\varphi_{0} \gg 1$ implies then

$$
\lambda \sinh \Delta=\frac{b}{b_{c}} \frac{1}{\theta} \tan \frac{\theta}{2} \ll \sqrt{\frac{1-\sin \frac{\theta}{2}}{1+\sin \frac{\theta}{2}}}<1 .
$$

Moreover, in order to have $0<\Delta<\infty$, eq. (5.32) implies that the angle $\theta$ has to lie in the range $0<\theta<\pi$ : this implies that $b / b_{c}$ has to be much smaller than a function of $\theta$ bounded by 2 , and thus small, as anticipated. We can now obtain the profile $\varphi(s)$ as

$$
s \simeq \int_{\tilde{\varphi}-\varphi(s)}^{\Delta} d x \frac{\sinh x}{\sqrt{\sinh ^{2} \Delta-\sinh ^{2} x}}=\arcsin \left(\tanh \Delta \sqrt{1-\left(\frac{\sinh (\tilde{\varphi}-\varphi(s))}{\sinh \Delta}\right)^{2}}\right)
$$

which inverted gives

$$
\sinh (\tilde{\varphi}-\varphi(s))=\tan \frac{\theta}{2} \sqrt{1-\left(\frac{\sin s}{\sin \frac{\theta}{2}}\right)^{2}}
$$

In order to obtain the effective action we still need to evaluate the integral $B\left(\varphi_{0}, \tilde{\varphi}\right)$, which in the given approximation reads

$$
B\left(\varphi_{0}, \tilde{\varphi}\right) \simeq \frac{1}{\lambda} \int_{0}^{\Delta} \frac{d x}{\sinh \Delta} e^{-x} \sqrt{1-\left(\frac{\sinh x}{\sinh \Delta}\right)^{2}}
$$

Setting $\cos \phi=\sinh x / \sinh \Delta$ the integral is easily evaluated, and gives

$$
B\left(\varphi_{0}, \tilde{\varphi}\right) \simeq \frac{\pi \alpha_{\mathrm{eff}}^{\prime} m \theta}{b}\left[\frac{\pi}{2}-\frac{\theta}{2 \sin ^{2} \frac{\theta}{2}}+\cot \frac{\theta}{2}\right] .
$$

In conclusion, we have for the effective action

$$
\left.S_{\mathrm{eff}, \mathrm{E}}\right|_{\varphi_{0} \gg 1}=2 \pi \alpha_{\mathrm{eff}}^{\prime} m^{2}\left[\theta \cot ^{2} \frac{\theta}{2}+\pi-\frac{\theta}{\sin ^{2} \frac{\theta}{2}}-2 \cot \frac{\theta}{2}\right]
$$

which at this level of approximation turns out to be independent of $b$, and of order $\mathcal{O}\left(\mathrm{m}^{2}\right)$. This is of course due to the fact that we are neglecting important subleading contributions: indeed, a logarithmic term $\propto \log \alpha_{\text {eff }}^{\prime} m / b$ would appear if we naively included the contribution coming from the $\tilde{\varphi}$ term in $f(\tilde{\varphi})$ (see eq. (5.21)). This term is of the same order of contributions neglected in the approximation above, and thus it is not consistent to include it; nevertheless, it shows how a non trivial dependence on $b$ could appear at subleading order. 


\subsubsection{Case $\lambda \gg 1$}

We determine now the explicit form of the solution in the case of large $\lambda$, which is expected to describe the small $\theta$ region, $\theta \ll b /\left(2 \pi \alpha_{\text {eff }}^{\prime} m\right)=2 b / b_{c} \leq 2$. According to eq. (5.20), $\Delta=\tilde{\varphi}-\varphi_{0}$ is of order $\mathcal{O}\left(\lambda^{-1}\right),{ }^{23}$ and so we can perform an expansion in powers of $\Delta$ of the relevant quantities.

We begin again by solving the equations (5.13) and (5.15) for the boundary values. Expanding eq. (5.13) as

$$
\cosh \tilde{\varphi}-\Delta \sinh \tilde{\varphi}+\frac{1}{2} \Delta^{2} \cosh \tilde{\varphi}+\mathcal{O}\left(\Delta^{3}\right)=\lambda \Delta \cosh \tilde{\varphi}\left[\cosh \tilde{\varphi}-\Delta \sinh \tilde{\varphi}+\mathcal{O}\left(\Delta^{2}\right)\right]
$$

we see that up to $\mathcal{O}\left(\Delta^{2}\right)$ we have

$$
(1-\lambda \Delta \cosh \tilde{\varphi})(\cosh \tilde{\varphi}-\Delta \sinh \tilde{\varphi})=\mathcal{O}\left(\Delta^{2}\right) .
$$

The term in the second pair of brackets is positive for $\Delta<1$, and of order $\mathcal{O}\left(\Delta^{0}\right)$, and so we infer

$$
\cosh \tilde{\varphi}=\frac{1}{\lambda \Delta}+\mathcal{O}\left(\Delta^{2}\right) .
$$

Expanding now eq. (5.15) we obtain

$$
\begin{aligned}
\frac{\theta}{2} & =\Delta \int_{0}^{1} d x \frac{x(\cosh \tilde{\varphi}-\Delta \sinh \tilde{\varphi})+\mathcal{O}\left(\Delta^{2}\right)}{\sqrt{1-x^{2}}(\cosh \tilde{\varphi}-\Delta \sinh \tilde{\varphi})+\mathcal{O}\left(\Delta^{2}\right)} \\
& =\Delta \int_{0}^{1} d x \frac{x}{\sqrt{1-x^{2}}}+\mathcal{O}\left(\Delta^{3}\right)=\Delta+\mathcal{O}\left(\Delta^{3}\right) .
\end{aligned}
$$

Summarising, we have the solution ${ }^{24}$

$$
\begin{aligned}
\cosh \tilde{\varphi} & =\frac{4 \pi \alpha_{\mathrm{eff}}^{\prime} m}{b}=\frac{b_{c}}{b}, \\
\Delta & =\frac{\theta}{2},
\end{aligned}
$$

and thus

$$
\varphi_{0}=\tilde{\varphi}-\Delta=\operatorname{arccosh} \frac{b_{c}}{b}-\frac{\theta}{2} .
$$

Since the left-hand side of eq. (5.44) is larger than one, in order to have a real solution we must satisfy

$$
\frac{b}{b_{c}} \leq 1
$$

which is exactly the general bound derived in the previous section, eq. (5.17). A more restrictive requirement is expected from $\varphi_{0} \geq 0$, which however yields

$$
\frac{b}{b_{c}} \leq \frac{1}{\cosh \frac{\theta}{2}}=1+\mathcal{O}\left(\theta^{2}\right)
$$

\footnotetext{
${ }^{23}$ Actually, from eq. (5.20), we can infer that $\Delta$ is of order $\mathcal{O}\left(\lambda^{-1-\epsilon}\right)$, with $\epsilon \geq 0$. The actual value $\epsilon=0$ comes out of the calculation.

${ }^{24}$ Due to the different boundary conditions, this solution is not expected to reduce to the one obtained in the previous section in the limit $\theta \rightarrow 0$.
} 
that at the given level of approximation is the same constraint found above. Of course, higher-order corrections are expected to modify this result. For completeness, we give also the explicit form of the profile, which is obtained by integrating

$$
s=\Delta \int_{\frac{\tilde{\tilde{\varphi}-\varphi(s)}}{\Delta}}^{1} d x \frac{x}{\sqrt{1-x^{2}}}=\Delta \sqrt{1-\left(\frac{\tilde{\varphi}-\varphi(s)}{\Delta}\right)^{2}}
$$

inverting this relation we obtain

$$
\tilde{\varphi}-\varphi(s)=\frac{\theta}{2} \sqrt{1-\left(\frac{2 s}{\theta}\right)^{2}}
$$

It is now easy to obtain the effective action, after we have computed one last integral, namely

$$
B\left(\varphi_{0}, \tilde{\varphi}\right)=\Delta \int_{0}^{1} d x \sqrt{1-x^{2}}[\cosh \tilde{\varphi}+\mathcal{O}(\theta)]=\frac{\theta \pi}{8} \cosh \tilde{\varphi}+\mathcal{O}\left(\theta^{2}\right) .
$$

Substituting $\tilde{\varphi}$ into the other two terms of (5.21) we finally obtain

$$
\left.S_{\mathrm{eff}, \mathrm{E}}\right|_{\lambda \gg 1}=\frac{b^{2}}{2 \pi \alpha_{\mathrm{eff}}^{\prime} \theta} \operatorname{arccosh} \frac{b_{c}}{b}+2 \pi^{2} \alpha_{\mathrm{eff}}^{\prime} m^{2}-\frac{2 b m}{\theta} \sqrt{\left(\frac{b_{c}}{b}\right)^{2}-1},
$$

up to order $\mathcal{O}\left(\theta^{0}\right)$.

The advantage of this approximation over the other one is that it extends up to "large" values of $b$, i.e., up to $b_{c}=4 \pi \alpha_{\text {eff }}^{\prime} m$. As we will discuss in detail in the next section, the physically interesting region in Minkowski space lies at large impact-parameter values, and an appropriate extension in $b$ beyond $b_{c}$ will be required: the expression obtained at large $\varphi_{0}$, which is valid only at small $b$, is not reliable for this purpose. Indeed, we see from eq. (5.44) that in order to perform this extension we have to pass through the value $\tilde{\varphi}=0$, which is clearly inconsistent with the assumption that $\varphi_{0}$ is large.

\subsubsection{Numerical results}

We discuss now briefly our numerical results. It is convenient to perform the numerical calculation by taking $\varphi_{0}$ and $\tilde{\varphi}$ as independent variables, and then calculate $b$ and $\theta$ as functions of $\varphi_{0}$ and $\tilde{\varphi}$ through eqs. (5.13) and (5.15). A minor drawback of this approach is that the space of parameters (i.e., $b$ and $\theta$ ) is not scanned uniformly. In all the figures $b$ is measured in units of $b_{c}=4 \pi \alpha_{\text {eff }}^{\prime} m$.

In figure 8 we show the curves of constant $\varphi_{0}$ in the $\theta-b$ plane, for various values of $\varphi_{0}$, and we compare the numerical results with the available analytic expressions. It is clear from this figure that the analytic results cover only a small portion of the region of $\theta-b$ plane where a real positive solution for $\varphi(s)$ exists. In the bulk of this region none of the two conditions $\lambda \gg 1$ and $\varphi_{0} \gg 1$ apply, so that we cannot use the approximations discussed above. Moreover, it turns out that for small enough $b$ there are solutions with $\theta>2 \pi$, or even larger: this means that there are several branches for the solution, corresponding to multiple "twists" of the helicoid. It is evident that the expression obtained for $\varphi_{0} \gg 1$ 


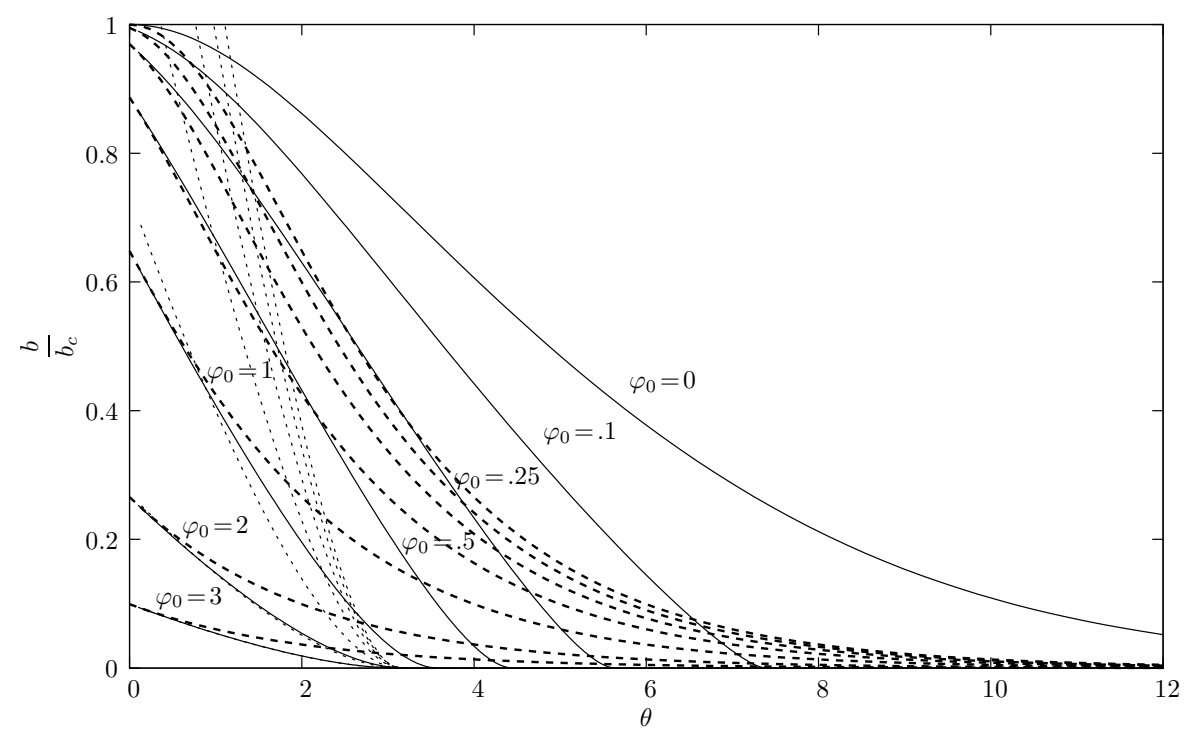

Figure 8. Curves of constant $\varphi_{0}$ in the $\theta-b$ plane. The solid lines represent the numerical results, the dotted lines represent the analytical result obtained in the case $\varphi_{0} \gg 1$, and the dashed lines represent the analytical result obtained in the case $\lambda \gg 1$.

is already a good approximation at $\varphi_{0}=2$, being practically indistinguishable from the numerical results. However, large values of $\varphi_{0}$ correspond to small values of $b$, and the approximation does not work in the region $b \sim b_{c}$, which will turn out to be physically relevant. On the other hand, in the region of small $\theta$ the expression obtained for $\lambda \gg 1$ is a good approximation in the whole range for $b$, up to $b=b_{c}$.

In figure 9 we show the curves of constant $\tilde{\varphi}$. Again, the large $-\varphi_{0}$ approximation works well only at small $b$, were it describes rather accurately the numerical results. The large $-\lambda$ approximation gives a constant value for $\tilde{\varphi}$ as a function of $\theta$, which however coincides with the small $-\theta$ limit of the numerical results in the whole $b$-range.

In figures 10 and 11 we show the results for the effective action, plotted as a function of $b$ for various values of $\theta$. The action is plotted in units of $8 \pi \alpha_{\text {eff }}^{\prime} m^{2}=2 b_{c} m$. The numerical results show that it is a monotonic function of $b$ and $\theta$, which moreover is bounded by the value $S_{\text {eff, E }}=2 \pi^{2} \alpha_{\text {eff }}^{\prime} m^{2}=\frac{\pi}{2} b_{c} m$. It turns out that, at fixed $\theta, S_{\text {eff, E vanishes at some }}$ point $b=\bar{b}(\theta)$, and it is positive for $b>\bar{b}(\theta)$. This simply means that, while for $b<\bar{b}(\theta)$ a connected helicoidal surface is more convenient, for $b>\bar{b}(\theta)$ it is less convenient than a disconnected configuration. As for the comparison with the analytic results, the large$\varphi_{0}$ approximation, which gives for the effective action a $b$-independent function, correctly describes the $b \rightarrow 0$ limit. On the other hand, the large $-\lambda$ approximation is a very good approximation for $S_{\text {eff, }}$ in the whole $b$-range for $\theta \lesssim 0.5$.

\section{Analytic continuation into Minkowski space-time}

In this section we want to discuss the physical predictions that can be obtained after analytic continuation of the Euclidean effective action. As already remarked, an approximate 


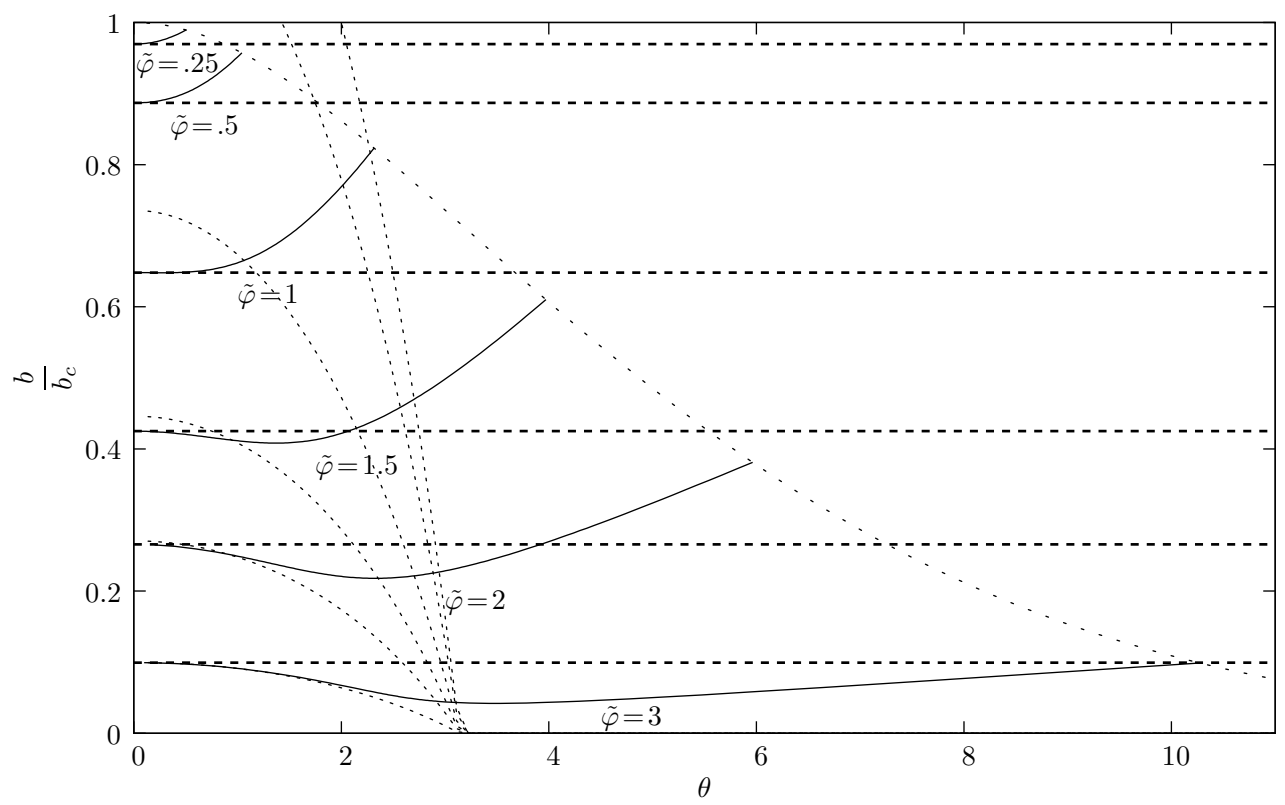

Figure 9. Curves of constant $\tilde{\varphi}$ in the $\theta-b$ plane. The key is as in figure 8 . The dotted line with sparse dots corresponds to the limiting case $\varphi_{0}=0$, i.e., $\tau(0)=0$, and is plotted for reference.

solution as the ones discussed in the previous section is not expected to capture the exact analytic form of the effective action. Although this is not rigorous from a mathematical point of view, we can nevertheless perform the analytic continuation into Minkowski space-time of our approximate expressions, and use physical arguments as a guide in order to judge the validity of the results obtained for the Reggeon-exchange amplitude. For instance, the expression obtained at large $\varphi_{0}$ becomes, after analytic continuation,

$$
\left.S_{\mathrm{eff}, \mathrm{E}}\right|_{\varphi_{0} \gg 1} \rightarrow \frac{i m b_{c}}{2}\left(\chi \operatorname{coth}^{2} \frac{\chi}{2}-\frac{\chi}{\sinh ^{2} \frac{\chi}{2}}-2 \operatorname{coth} \frac{\chi}{2}-i \pi\right),
$$

which is essentially an energy-dependent phase, so that the resulting impact-parameter amplitude is an oscillating function of energy. However, as we have explained in the previous section, this result corresponds to a region in which the impact parameter $b$ is very small, namely $b \ll b_{c}=4 \pi \alpha_{\text {eff }}^{\prime} m$, and since $b_{c} \rightarrow 0$ when $m \rightarrow 0$, its contribution to the scattering amplitude would vanish as $m^{2}$ at fixed $\chi$, thus not allowing for a suitable limit of zero quark mass. Moreover, the flat-space approximation, described in section 2, which we are using for the calculation of the minimal surface, is not expected to be reliable in the region of small $b$.

As we discuss below, relevant contributions to the physical scattering amplitude, i.e., after analytic continuation to Minkowski space, come from the region of $b>b_{c}$, in such a way that the result has a non-zero limit when $m \rightarrow 0$. Hence, a more significant result is obtained starting from the solution obtained at large $\lambda$, which as we have explained describes the whole $b \leq b_{c}$ region at small $\theta$, and which is therefore more suitable for an extension to larger values of $b$. This is supported by the comparison with our numerical results for 


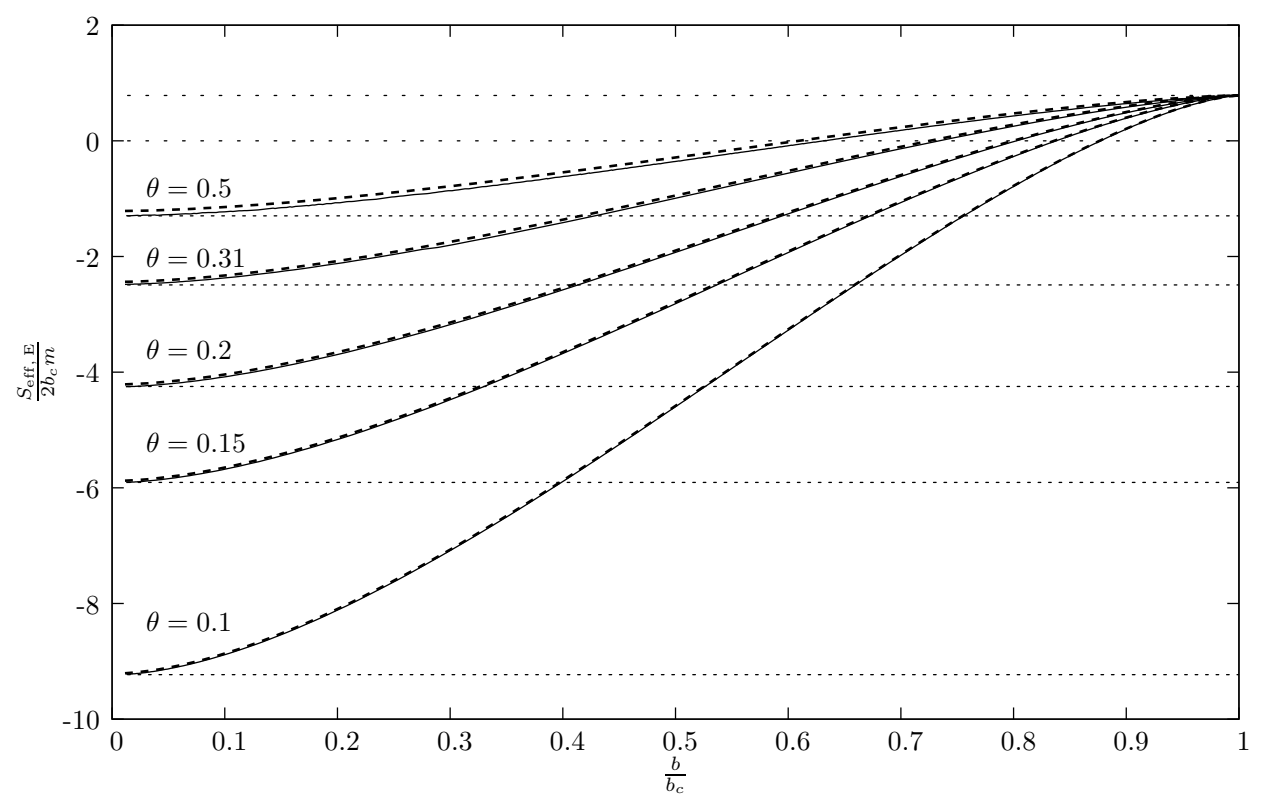

Figure 10. Effective action as a function of $b$ for various values of $\theta$ : small $\theta$. The action is plotted in units of $2 b_{c} m=8 \pi \alpha_{\mathrm{eff}}^{\prime} m^{2}$. The key is as in figure figure 8. The values 0 and $\pi / 4$ are plotted for reference with a dotted line with sparse dots.

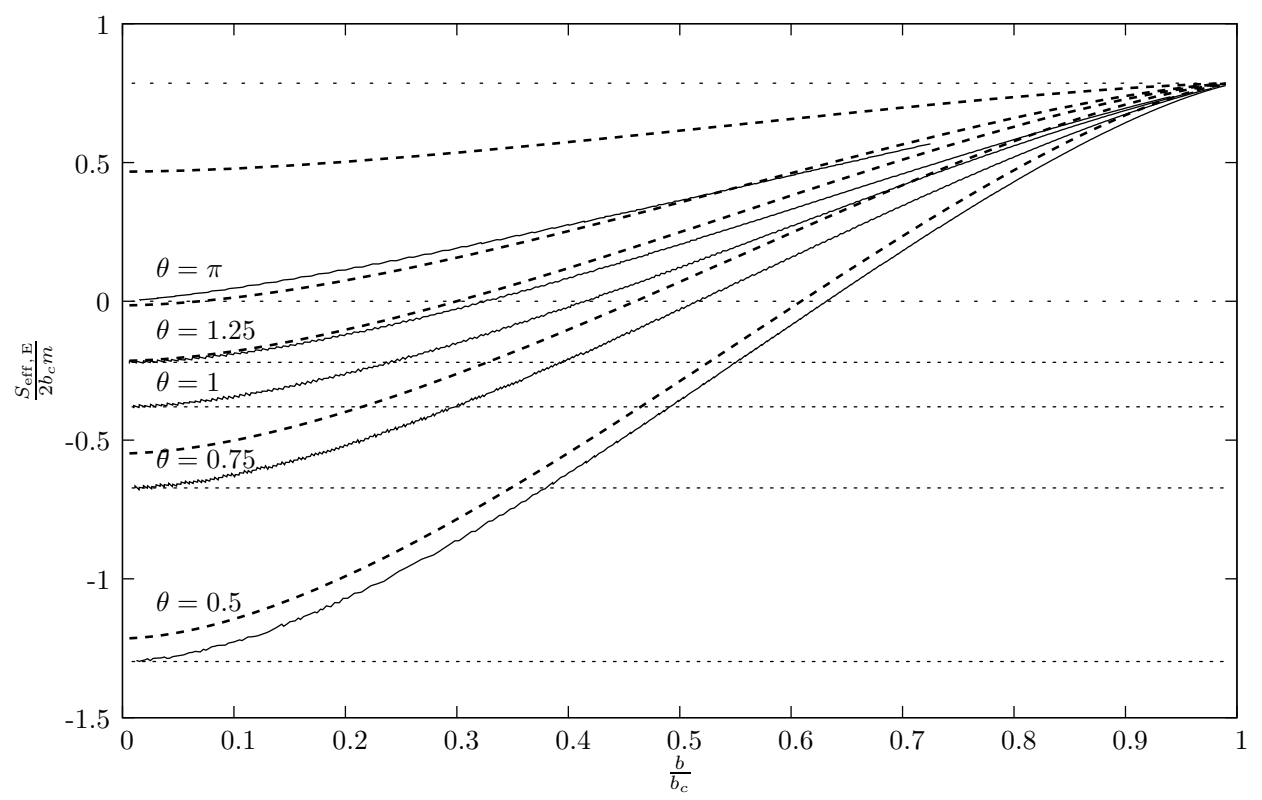

Figure 11. Effective action as a function of $b$ for various values of $\theta$ : "large" $\theta$. The key is as in figure figure 8 . The values 0 and $\pi / 4$ are plotted for reference with a dotted line with sparse dots.

the Euclidean effective action (figures 10 and 11), which shows a fairly good agreement with the large $-\lambda$ analytic expression as regards the dependence on $b$. In particular, the agreement improves as $b$ tends towards $b_{c}$. 


\subsection{Subcritical region $b \leq b_{c}$}

Making then the substitution $\theta \rightarrow-i \chi$ in eq. (5.52), we obtain

$$
\left.S_{\mathrm{eff}, \mathrm{E}}\right|_{\lambda \gg 1} \rightarrow S_{\mathrm{eff}, \mathrm{M}}=i \frac{b^{2}}{2 \pi \alpha_{\mathrm{eff}}^{\prime} \chi} \operatorname{arccosh} \frac{b_{c}}{b}-i \frac{2 b m}{\chi} \sqrt{\left(\frac{b_{c}}{b}\right)^{2}-1}+2 \pi^{2} \alpha_{\mathrm{eff}}^{\prime} m^{2} .
$$

The real part of expression eq. (6.2) consists simply of a $b, \chi$-independent term, while the whole $b, \chi$-dependence is contained in terms which are purely imaginary for $b \leq b_{c}$, and which moreover are vanishing in the limit $\chi \rightarrow \infty$. As we show in appendix $C$, the phases $\Phi\left(\mathcal{C}_{\vec{u}_{i}}\right)$ in the contribution of the spin factor remain real in this region after analytic continuation, and they are independent of energy. Therefore, in the region $b \leq b_{c}$, corresponding to the region where a real solution exists in Euclidean space, the impactparameter amplitude is an oscillating function. However, this region -which we can call the core region- has an energy-independent size, so that its contribution at small quark mass $m$ is of the order of ${ }^{25}$

$$
\left|\mathcal{A}^{\text {core }}\right| \propto\left|\int_{0}^{b_{c}} d b b J_{0}(b q) e^{-i \operatorname{Im} S_{\mathrm{eff}, \mathrm{M}}} e^{-2 \pi^{2} \alpha_{\mathrm{eff}}^{\prime} m^{2}} e^{i \Phi_{\mathrm{spin}}}\right| \leq \frac{b_{c}^{2}}{2}=\mathcal{O}\left(m^{2}\right),
$$

and thus vanishing in the limit $m \rightarrow 0$. Therefore, as anticipated, the relevant contributions to the amplitude come from the region $b>b_{c}$ : in the remaining part of this section we discuss how this region can be reached, and how expression eq. (6.2) is modified.

\subsection{Analytic continuation towards $b>b_{c}$}

As we have already pointed out, a real solution of the saddle-point equation in Euclidean space exists only in a limited range of impact-parameter values. The limitation to real solutions is dictated by the fact that the path-integral eq. (3.3) is over real paths $\mathcal{C}_{ \pm}$in Euclidean space, leading in turn to an integral over real $\tau^{ \pm}$. The limitation $b \leq b_{c}$ can be seen also in the effective action, since $b_{c}$ is a branch point for this quantity, beyond which it acquires an imaginary component. Nevertheless, since we are mainly interested in the impact-parameter amplitude in Minkowski space, which is generally a complex quantity, we can think of extending the result beyond $b_{c}$, leaving aside the limitations coming from the requirement of reality, by making use of an appropriate analytic continuation. To justify this procedure from a mathematical point of view we can invoke analyticity in the impact parameter, which allows us to determine the value of the impact-parameter amplitude for $b>b_{c}$ up to fixing the ambiguity in the choice of the Riemann sheet.

As we have said above, $b_{c}$ is a branch point for $S_{\text {eff, }}$, and so we need to specify a prescription in order to go from $b<b_{c}$ to $b>b_{c}$. To this extent, we choose the usual

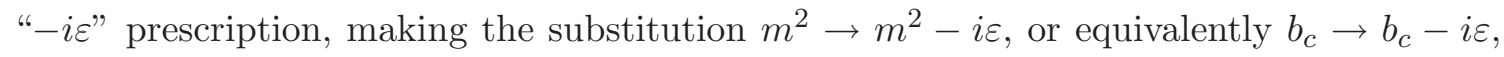
in eq. (6.2). Defining $y=b_{c} / b$, this prescription amounts to going from $y>1$ to $y<1$

\footnotetext{
${ }^{25}$ For $b \lesssim R_{0}$ one should also include corrections due to the curvature, which cannot be neglected for small impact parameter. The corresponding contribution to the amplitude is however limited by the unitarity bound on the impact-parameter amplitude, and it is subleading with respect to contributions from the "tail", i.e., from $b>b_{c}$, discussed below.
} 
passing in the lower half of the complex $y$-plane, so that the phase of $y-1$ goes from $-\varepsilon$ to $-\pi+\varepsilon$. We have then

$$
\begin{aligned}
& \sqrt{y^{2}-i \varepsilon-1} \underset{y>1 \rightarrow y<1}{\rightarrow}-i \sqrt{1-y^{2}}, \\
& \operatorname{arccosh} y \underset{y>1 \rightarrow y<1}{\rightarrow}-i \arccos y
\end{aligned}
$$

and therefore the effective action becomes for $b>b_{c}$

$$
S_{\mathrm{eff}, \mathrm{M}} \rightarrow \frac{b^{2}}{2 \pi \alpha_{\mathrm{eff}}^{\prime} \chi} \arccos \frac{b_{c}}{b}-\frac{2 b m}{\chi} \sqrt{1-\left(\frac{b_{c}}{b}\right)^{2}}+2 \pi^{2} \alpha_{\mathrm{eff}}^{\prime} m^{2} .
$$

The effective action is then real at $b>b_{c}$; moreover, for very large $b \gg b_{c}$ the expression simplifies to

$$
S_{\mathrm{eff}, \mathrm{M}} \simeq \frac{b^{2}}{4 \alpha_{\mathrm{eff}}^{\prime} \chi}-\frac{4 b m}{\chi}+2 \pi^{2} \alpha_{\mathrm{eff}}^{\prime} m^{2},
$$

which yields then a Gaussian-like impact-parameter amplitude. The results eqs. (6.5) and (6.6) represent the main physical output of our calculation of the Reggeon-exchange amplitude, as anticipated in the Introduction. ${ }^{26}$

Equations (6.5) and (6.6) call for comments. The large $-b$ expansion of eq. (6.6) can be equivalently seen as a small- $m$ expansion, up to order $\mathcal{O}\left(m^{2}\right) .{ }^{27}$ In particular, taking $m$ to zero we obtain the same result of [14], which corresponds to a complex constant Euclidean profile $\tau(\sigma) \equiv-i b / \theta$, i.e., $\varphi(s) \equiv-i \pi / 2$. As we will show in the next section, taking the Fourier transform with respect to $\vec{b}$ one obtains for the amplitude a Regge-pole behaviour $\mathcal{A}_{\mathcal{R}} \propto s^{\alpha_{\mathcal{R}}(t)}$, with a linear Reggeon trajectory $\alpha_{\mathcal{R}}(t)=\alpha_{\text {eff }}^{\prime}{ }^{t}$ with intercept $\alpha_{0}=0 .{ }^{28}$ In [14] also the effect of quadratic fluctuations of the world-sheet around the classical solution were considered, which yielded a contribution $\delta \alpha_{0}=n_{\perp} / 24$ to the Reggeon intercept, with $n_{\perp}$ the number of transverse directions in which the string could fluctuate. In this paper we do not have computed quantum fluctuations, which require more work due to the non trivial form of the classical solution.

The possibility to take the small- $m$ limit seems to be in contradiction with our previous remarks on the domain of applicability of our approximation. We recall that the flat-space approximation is expected to be valid for $b \geq R_{0}$, which is the scale at which the linear potential sets in, and that a real solution in Euclidean space exists for $b \leq b_{c}=4 \pi \alpha_{\text {eff }}^{\prime} m$. Setting $\Lambda_{\mathrm{QCD}}^{-2}=4 \pi \alpha_{\text {eff }}^{\prime}$, and $R_{0}=\kappa \Lambda_{\mathrm{QCD}}^{-1}$, with $\kappa$ of order 1 , we have then that $b$ has to be in the window $\kappa \leq b \Lambda_{\mathrm{QCD}} \leq m \Lambda_{\mathrm{QCD}}^{-1}$, which disappears when $m \Lambda_{\mathrm{QCD}}^{-1}<\kappa$ (see figure 12 left). However, we have shown that, after analytic continuation $\theta \rightarrow-i \chi$ to Minkowski space, it is possible to further extend the Reggeon-exchange amplitude to larger values of $b$, i.e., to $b>m \Lambda_{\mathrm{QCD}}^{-1}$, by means of analytic continuation in $b$ (see figure 12 right). In this

\footnotetext{
${ }^{26}$ The term neglected in eq. (3.19) would give an extra contribution $\delta S_{\text {eff, } \mathrm{M}}=\delta c(4 b / \chi) \sqrt{1-\left(b_{c} / b\right)^{2}}$ to eq. (6.5). For large $b$ this contribution is approximately $\delta S_{\text {eff, } \mathrm{M}} \simeq \delta c(4 b / \chi)$.

${ }^{27}$ More precisely, up to order $\mathcal{O}\left(\alpha_{\text {eff }}^{\prime} m^{2}\right)$.

${ }^{28}$ Our expression for the spin factor is enhanced by a factor of $s$ with respect to the one found in [14], which would apparently raise the intercept by 1 . However, an extra suppressing factor $s^{-1}$ appears when taking properly into account the fact that the quarks and antiquarks are partons inside of mesons [69].
} 

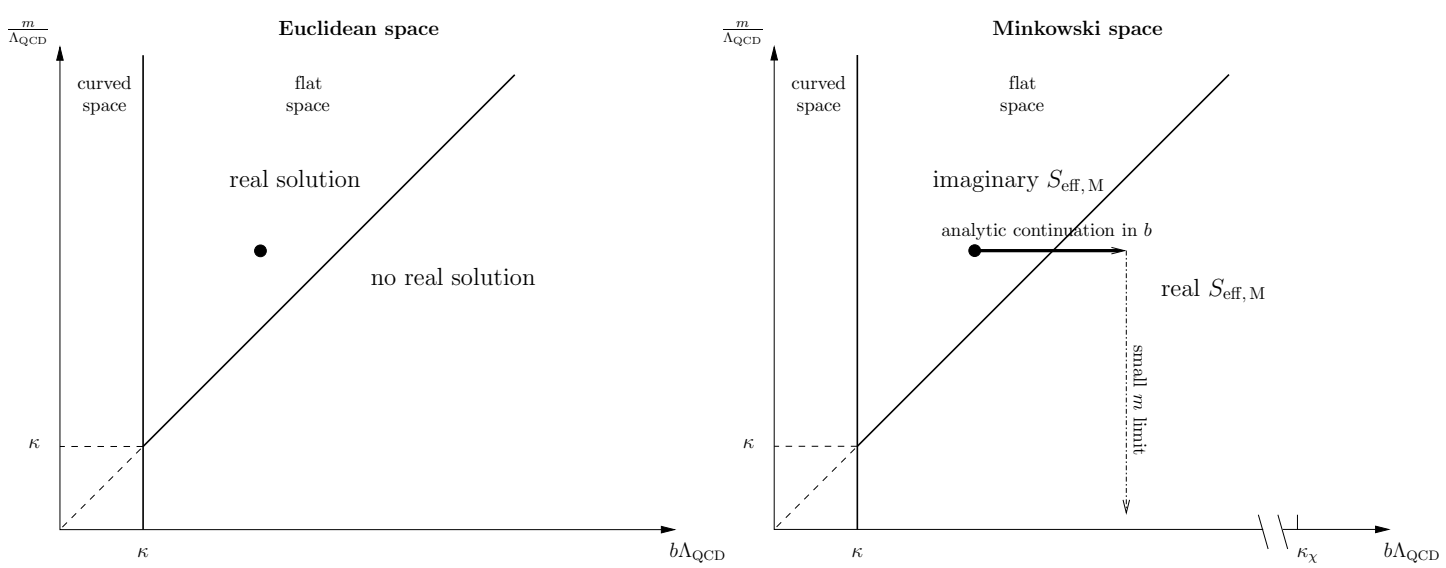

Figure 12. Analytic continuation in the ( $b, m)$ plane (in dimensionless units).

Euclidean space (left). Vertical line at $b=R_{0}=\kappa \Lambda_{\mathrm{QCD}}^{-1}$ : separates the region where the relevant geometry is essentially flat $\left(b \Lambda_{\mathrm{QCD}} \geq \kappa\right)$ from the one where the curvature cannot be neglected. Tilted straight line $b \Lambda_{\mathrm{QCD}}=m \Lambda_{\mathrm{QCD}}^{-1}$ : a real solution to the variational problem exists above this line. Together with the vertical line it defines the "wedge" where our approximation is valid.

Minkowski spacetime (right). After analytic continuation $\theta \rightarrow-i \chi$, which connects the black dots, it is possible to perform a further analytic continuation in $b$, which allows then to take the small- $m$ limit. The region of $b \Lambda_{\mathrm{QCD}}$ relevant to Reggeon exchange extends (approximately) up to the value $\kappa_{\chi}=\sqrt{\chi}$.

region, which is connected to the axis $m=0$, the Minkowskian effective action becomes real, and there is no further obstruction (at least at the given level of approximation) to take the limit $m \rightarrow 0$.

We have then shown that in order to obtain rigorously a non-zero result in the singular $m=0$ case, one needs to start from $m \neq 0$ and then perform an analytic continuation in the impact parameter $b$ beyond the branch point $b_{c}$ : indeed, the contribution from the region $b \leq b_{c} \propto m$ is proportional to $m^{2}$ at high energy and in the limit $m \rightarrow 0$, and thus the amplitude would vanish. ${ }^{29}$ Following eq. (6.6), the impact-parameter region giving the major contribution to the Regge amplitude extends to $b \rightarrow \sqrt{\alpha_{\text {eff }}^{\prime} \chi} \gg \alpha_{\text {eff }}^{\prime} m$ (cfr. $\kappa_{\chi} \gg \kappa$ in figure 12 right).

The result obtained through analytic continuation in $\theta$ of the small- $\theta$ solution (which is, strictly speaking, a large $-\lambda$ solution), and the successive analytic continuation in $b$ past the branch-point $b_{c}$, is then sensible from the physical point of view. Nevertheless, two important analyticity issues are present.

- The terms that we have neglected contain higher positive powers of $\theta$, and so, although small in Euclidean space, could give important, and in principle also dominant contributions to the amplitude after analytic continuation. Indeed, terms of order $\mathcal{O}\left(\theta^{n}\right)$ would give larger and larger $\mathcal{O}\left(\chi^{n}\right)$ terms as $n$ increases, which could possibly lead to violations of the Froissart bound [73-75]. However, to correctly perform

\footnotetext{
${ }^{29}$ More precisely, it would reduce to the "curved" contribution from the region $b \lesssim R_{0}$, which is however not under control at the present stage, but which is subleading in energy with respect to the "tail" contribution.
} 
the analytic continuation one should first resum all orders in $\theta$, which amounts to obtain the exact solution in explicit form, and only after that take $\theta \rightarrow-i \chi$. This could easily lead to modifications of the Minkowskian effective action which at large $\chi$ become irrelevant. ${ }^{30}$

- In order to fix the ambiguity of the analytic continuation in $b$, we have chosen the

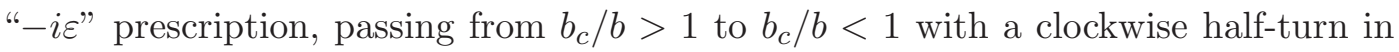
the complex $b_{c} / b$-plane. The correctness of this choice is clear from a physical point of view: indeed, if we had chosen the opposite prescription, i.e., if we had passed from $b_{c} / b>1$ to $b_{c} / b<1$ moving in the upper half of the complex plane, we would have obtained an unphysical, divergent impact-parameter amplitude at large $b$. However, a completely satisfactory explanation from a mathematical point of view is lacking at the moment. It is possible that the " $-i \varepsilon$ " prescription would turn out naturally by taking into account the exact dependence on $\theta$ in the Euclidean effective action. ${ }^{31}$ Another interesting possibility, which we consider in the next section, is that the whole multi-sheet structure of the Minkowskian effective action has physical relevance.

These delicate analyticity problems are currently open, and require further work to be solved. Nevertheless, although the results cannot be taken too "literally", it is interesting to investigate the possible physical consequences of eq. (6.5), in particular the effects of a small fermion mass on the Reggeon singularity.

\section{The Reggeon amplitude}

It is interesting to investigate the effects of a small fermion mass on the Reggeon singularity, computing the Reggeon-exchange amplitude eq. (3.1) by performing the Fourier transform of the impact-parameter amplitude, namely

$$
\mathcal{A}_{\mathcal{R}}\left(s, t=-q^{2}\right) \equiv-2 i s \int d^{2} b e^{i \vec{q} \cdot \vec{b}} a(\vec{b}, \chi)=-4 i \pi s \int_{0}^{\infty} d b b J_{0}(q b) a(b, \chi),
$$

where in the last passage we have used azimuthal invariance, and with a small abuse of notation we have denoted $a(\vec{b}, \chi)=a(b, \chi)$.

The impact-parameter amplitude is given by the product of several factors. The first factor is the contribution $e^{-S_{\text {eff, M }}}$ of the saddle point, which up to order $\mathcal{O}(m)$ reads (see also figure 13)

$$
e^{-S_{\mathrm{eff}, \mathrm{M}}}=e^{-\frac{b^{2}}{4 \alpha_{\mathrm{eff}}^{\prime} \chi}}\left(1+\frac{4 b m}{\chi}\right)+\mathcal{O}\left(m^{2}\right)
$$

\footnotetext{
${ }^{30}$ As an illustrative example, one could find that the $1 / \theta$ factor in eq. (5.52) is modified to $g(\theta)=$ $1 / \theta\left[1+\theta^{2} /(2+\cos \theta)\right]$. While the small- $\theta$ expansion gives $g(\theta) \simeq 1 / \theta+\theta / 3+\mathcal{O}\left(\theta^{2}\right)$, which is compatible with our result, the analytic continuation $\theta \rightarrow-i \chi$ would lead to $g(-i \chi)=i / \chi-i \chi /(2+\cosh \chi)$, which reduces to $g(-i \chi) \simeq i / \chi$ for $\chi \rightarrow \infty$, and thus would not change our result for the Regge trajectory.

${ }^{31}$ In order to have this prescription built in the exact expression for the Euclidean effective action, $b_{c}$ should appear multiplied by an appropriate function of $\theta$ : such a function must tend to 1 as $\theta \rightarrow 0$, and it should have a small negative imaginary component when $\theta \rightarrow-i \chi+\varepsilon$.
} 
A second factor is the contribution of the spin factors, evaluated at the saddle point and contracted with the bispinors corresponding to the interacting quarks and antiquarks. As we show in appendix $\mathrm{C}$, the calculation of this contribution can be performed exactly, but the result contains an implicit dependence on $\chi$ and $b$ which we have not been able to determine explicitly in the general case. We have obtained an explicit expression in the large $-\lambda$ approximation, as we have done for the effective action, but a comparison with numerical results shows that in this case the extrapolation of the analytic result to the region $b>b_{c}$ cannot be trusted. Nevertheless, spin effects are not expected to affect the behaviour of the Reggeon trajectory. For this reason, we have preferred not to include the spin factor in our analysis, delaying a detailed study to a future publication.

Two other factors should in principle be included, namely the contributions from the string fluctuations around the minimal surface, i.e., the factor $\mathcal{F}$ in eq. (3.9), and the contribution of quadratic fluctuations of the floating boundary around the saddle-point. At the present stage these contributions are not known (except for $\mathcal{F}$ in the case $m=0$, where it is $\left.\mathcal{F}\right|_{m=0} \propto s^{\frac{n_{\perp}}{24}}$ ), and they could easily introduce further dependence on $b$ and $\chi$, thus modifying the form of the impact-parameter amplitude.

However, an implicit assumption of the saddle-point approximation was that these contributions are not of exponential type, and so the term $e^{-S_{\text {eff, } \mathrm{M}}}$ will not change if the approximation method works. On the other hand, power-like factors are not completely under control; the same happens for the overall power of $s$, and for logarithmic prefactors $\chi \sim \log s$. It is therefore sensible, in a first approximation, to consider only the contribution eq. (7.2) from the saddle-point, ignoring all the other factors, and to determine the Reggeon trajectory in this case. Clearly, an overall factor $s^{\delta \alpha}$ would simply change the value of the intercept of an amount $\delta \alpha$. Moreover, the presence of factors $b^{n_{b}}$ in the impact-parameter amplitude, or logarithmic $\chi^{n_{\chi}}$ prefactors (with $n_{b}, n_{\chi}$ positive integers), would modify the nature of the singularity but not the Reggeon trajectory. We will discuss this issue in detail in subsection 7.2. As a final remark, notice that the extra factor of $s$ in front of the Fourier transform in eqs. (3.1) and (7.1) is cancelled by a compensating factor $s^{-1}$, which appears when taking properly into account the fact that the quarks and antiquarks are partons inside of mesons [69], as already mentioned in the previous section.

\subsection{The Reggeon singularity and small quark-mass effects}

In a first approximation, we therefore consider the following expression for the Reggeonexchange amplitude,

$$
\begin{aligned}
\mathcal{A}_{\mathcal{R}}(s, t) & \approx \frac{1}{2 \alpha_{\mathrm{eff}}^{\prime} \chi} \int_{0}^{\infty} d b b e^{-\frac{b^{2}}{4 \alpha_{\mathrm{e}}^{\prime} \chi}}\left(1+\frac{4 b m}{\chi}\right) J_{0}(q b)+\mathcal{O}\left(m^{2}\right) \\
& =\mathcal{T}_{0}(\chi, t)+m \mathcal{T}_{1}(\chi, t)+\mathcal{O}\left(m^{2}\right)
\end{aligned}
$$

where we are ignoring all numerical prefactors and the dependence on spin, which are actually irrelevant for the following discussion. Moreover, in eq. (7.3) we have introduced 


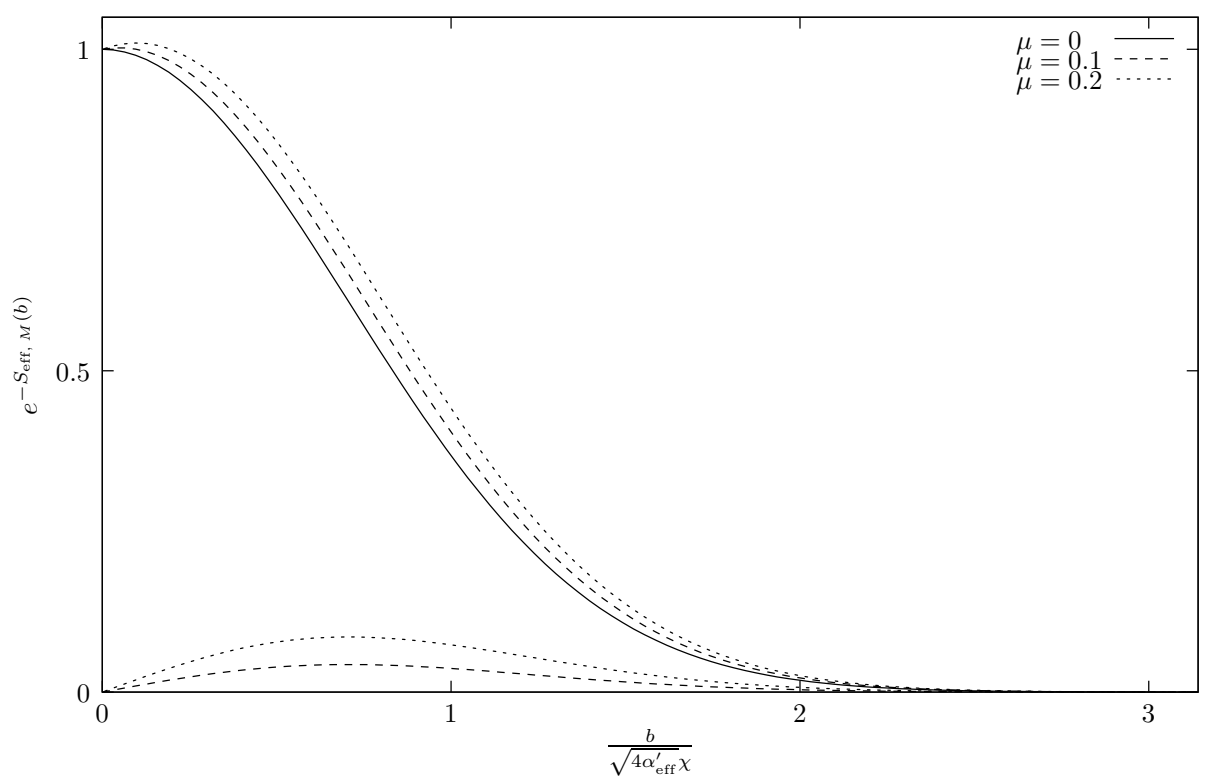

Figure 13. Impact-parameter amplitude at small $m$. Plot of the full saddle-point contribution $e^{-S_{\text {eff }, M}}$ in eq. (7.2) to the impact-parameter amplitude (upper curves) and of the mass-dependent term alone (lower curves) for different values of $\mu=8 m \sqrt{\alpha_{\mathrm{eff}}^{\prime} / \chi}$.

the quantities

$$
\begin{aligned}
\mathcal{T}_{0}(\chi, t) & =e^{-\alpha_{\text {eff }}^{\prime} \chi q^{2}} \\
\mathcal{T}_{1}(\chi, t) & =4 \sqrt{\frac{\pi \alpha_{\text {eff }}^{\prime}}{\chi}}\left\{\tilde{I}_{0}\left(\alpha_{\text {eff }}^{\prime} \chi \frac{q^{2}}{2}\right)-\alpha_{\text {eff }}^{\prime} \chi q^{2}\left[\tilde{I}_{0}\left(\alpha_{\text {eff }}^{\prime} \chi \frac{q^{2}}{2}\right)-\tilde{I}_{1}\left(\alpha_{\text {eff }}^{\prime} \chi \frac{q^{2}}{2}\right)\right]\right\} \\
& =8 \sqrt{\pi \alpha_{\text {eff }}^{\prime}} \frac{\partial}{\partial \chi}\left[\sqrt{\chi} \tilde{I}_{0}\left(\alpha_{\text {eff }}^{\prime} \chi \frac{q^{2}}{2}\right)\right],
\end{aligned}
$$

where $\tilde{I}_{n}(z) \equiv e^{-z} I_{n}(z)$, with $I_{n}(z)$ the modified Bessel functions. The factor $\left(2 \alpha_{\text {eff }}^{\prime} \chi\right)^{-1}$ has been inserted "by hand" in order to remove an extra logarithmic prefactor, and to fix (arbitrarily) the normalisation. As explained above, such prefactors are not completely under control, but they do not change the Reggeon trajectory. It is therefore sensible to start from the simpler "basic" expression without any extra power of $\chi$; the extension to the more general case is discussed in the next subsection.

As a first remark, notice that the slope of the amplitude at $t=0$, given by

$$
\frac{\partial \mathcal{A}_{\mathcal{R}}}{\partial t}(s, t=0)=\alpha_{\mathrm{eff}}^{\prime}\left(\chi+6 m \sqrt{\pi \alpha_{\mathrm{eff}}^{\prime} \chi}\right)
$$

is increased by the effect of the quark mass. Moreover, the dependence of the slope on energy is stronger when $m \neq 0$. These effects are related to the effective increase of the width of the impact-parameter amplitude, which can be seen in figure 13.

To uncover the nature of the Reggeon singularity we compute the Mellin transform of the amplitude. If we write the amplitude as $\mathcal{A}_{\mathcal{R}}(s, t)=\mathcal{A}(\chi, t)$, with $\chi \simeq \log \left(s / m_{1} m_{2}\right)$ at 
large energy, we can conveniently express the Mellin transform as an integral over $\chi$, i.e.,

$$
\mathcal{A}^{(M)}(\omega, t)=\int_{0}^{\infty} d \chi e^{-\omega \chi} \mathcal{A}(\chi, t) .
$$

The Mellin transform is clearly linear, and moreover it has the following properties,

$$
\begin{aligned}
(\chi f)^{(M)}(\omega) & =-\frac{\partial}{\partial \omega} f^{(M)}(\omega), \\
\left(\frac{\partial}{\partial \chi} f\right)^{(M)}(\omega) & =\omega f^{(M)}(\omega),
\end{aligned}
$$

which will be useful in the following. We thus write, discarding the subleading $\mathcal{O}\left(\mathrm{m}^{2}\right)$ terms,

$$
\mathcal{A}^{(M)}(\omega, t)=\mathcal{T}_{0}^{(M)}(\omega, t)+m \mathcal{T}_{1}^{(M)}(\omega, t) .
$$

The first term is easily evaluated, and yields

$$
\mathcal{T}_{0}^{(M)}(\omega, t)=\frac{1}{\omega+\alpha_{\mathrm{eff}}^{\prime} q^{2}}=\frac{1}{\omega-\alpha_{\mathrm{eff}}^{\prime} t} .
$$

This is the only term when $m=0$, and it clearly corresponds to a simple pole at $\omega=\alpha_{\text {eff }}^{\prime} t$; the corresponding Reggeon trajectory is linear, as found in [14]. Partially anticipating the discussion of the next subsection, we easily determine the effect of logarithmic prefactors on this term: exploiting the first property in eq. (7.7), an overall prefactor $\chi^{n}$ would simply transform the simple pole in eq. (7.9) into an $n$-th order pole, without changing its position.

In order to evaluate the second term,

$$
\mathcal{T}_{1}^{(M)}(\omega, t)=8 \sqrt{\pi \alpha_{\mathrm{eff}}^{\prime}} \omega \int_{0}^{\infty} d \chi e^{-\omega \chi} \sqrt{\chi} \tilde{I}_{0}\left(\alpha_{\mathrm{eff}}^{\prime} \chi \frac{q^{2}}{2}\right),
$$

we exploit the integral representation for the modified Bessel functions, which allows to write for $\tilde{I}_{0}$

$$
\tilde{I}_{0}(z)=\frac{2}{\pi} \int_{0}^{\frac{\pi}{2}} d \phi e^{-2 z \sin ^{2} \phi}
$$

and thus

$$
\mathcal{T}_{1}^{(M)}(\omega, t)=8 \sqrt{\pi \alpha_{\mathrm{eff}}^{\prime}} \omega \frac{2}{\pi} \int_{0}^{\frac{\pi}{2}} d \phi\left(\omega+\alpha_{\mathrm{eff}}^{\prime} q^{2} \sin ^{2} \phi\right)^{-\frac{3}{2}} \int_{0}^{\infty} d \chi \sqrt{\chi} e^{-\chi} .
$$

The $\chi$ integral is now easily evaluated, and yields $\int_{0}^{\infty} d \chi \sqrt{\chi} e^{-\chi}=\frac{\sqrt{\pi}}{2}$, and so

$$
\begin{aligned}
\mathcal{T}_{1}^{(M)}(\omega, t)= & -16 \sqrt{\alpha_{\mathrm{eff}}^{\prime}} \omega \frac{\partial}{\partial \omega} \omega^{-\frac{1}{2}} \int_{0}^{\frac{\pi}{2}} d \phi\left(1+\frac{\alpha_{\mathrm{eff}}^{\prime} q^{2}}{\omega} \sin ^{2} \phi\right)^{-\frac{1}{2}}= \\
& -16 \sqrt{\alpha_{\mathrm{eff}}^{\prime}} \omega \frac{\partial}{\partial \omega} \omega^{-\frac{1}{2}} K\left(-\frac{\alpha_{\mathrm{eff}}^{\prime} q^{2}}{\omega}\right),
\end{aligned}
$$

where $K(z)$ is the complete elliptic integral of the first kind (evaluated at a negative argument; see e.g. [76]). All in all, we have

$$
\mathcal{A}^{(M)}(\omega, t)=\frac{1}{\omega-\alpha_{\mathrm{eff}}^{\prime} t}-16 \sqrt{\alpha_{\mathrm{eff}}^{\prime}} m \omega \frac{\partial}{\partial \omega} \omega^{-\frac{1}{2}} K\left(\frac{\alpha_{\mathrm{eff}}^{\prime} t}{\omega}\right) .
$$


Since $t<0$ in the physical $s$-channel, this function is regular for all $\omega>0$, and it has a branch-point singularity at $\omega=0$. Analytically continuing in $t$ to $t>0$, i.e., to the physical $t$-channel, the Reggeon singularity moves on the positive real half-axis. As we have already said, the first term is a pole at $\alpha_{\text {eff }}^{\prime} t$. Also the second term is singular at $\alpha_{\text {eff }}^{\prime} t$, the singularity being governed by the behaviour of the elliptic integral near 1,

$$
K(z) \underset{z \rightarrow 1}{\simeq} \frac{1}{2} \log \frac{16}{1-z} .
$$

Explicitly,

$$
\begin{aligned}
\omega \frac{\partial}{\partial \omega} \omega^{-\frac{1}{2}} K\left(\frac{\alpha_{\mathrm{eff}}^{\prime} t}{\omega}\right) \underset{\omega \rightarrow \alpha_{\mathrm{eff}}^{\prime} t}{\simeq}-\frac{1}{2} \omega^{-\frac{1}{2}}\left\{\frac{1}{2} \log \frac{16 \omega}{\omega-\alpha_{\mathrm{eff}}^{\prime} t}+\frac{\alpha_{\mathrm{eff}}^{\prime} t}{\omega-\alpha_{\mathrm{eff}}^{\prime} t}\right\} \\
\underset{\omega \rightarrow \alpha_{\mathrm{eff}}^{\prime} t}{\simeq}-\frac{1}{2\left(\alpha_{\mathrm{eff}}^{\prime} t\right)^{\frac{1}{2}}}\left\{\frac{1}{2} \log \frac{16 \alpha_{\mathrm{eff}}^{\prime} t}{\omega-\alpha_{\mathrm{eff}}^{\prime} t}+\frac{\alpha_{\mathrm{eff}}^{\prime} t}{\omega-\alpha_{\mathrm{eff}}^{\prime} t}\right\},
\end{aligned}
$$

so that putting everything together we have

$$
\mathcal{A}^{(M)}(\omega, t) \underset{\omega \rightarrow \alpha_{\mathrm{eff}}^{\prime} t}{\simeq} \frac{1+8 \alpha_{\mathrm{eff}}^{\prime} m t^{\frac{1}{2}}}{\omega-\alpha_{\mathrm{eff}}^{\prime} t}+4 m t^{-\frac{1}{2}} \log \frac{16 \alpha_{\mathrm{eff}}^{\prime} t}{\omega-\alpha_{\mathrm{eff}}^{\prime} t} .
$$

The leading singularity of $\mathcal{A}^{(M)}$ is then a pole at $\omega=\alpha_{\text {eff }}^{\prime} t$, with residue (up to numerical factors)

$$
\text { Res }=1+8 \alpha_{\text {eff }}^{\prime} t^{\frac{1}{2}} m
$$

Moreover, there is a logarithmic branch-point singularity at $\omega=\alpha_{\mathrm{eff}}^{\prime} t$ due to the second term of $\mathcal{A}^{(M)}$. At $t=0$ this singularity becomes an algebraic one, since in that case $\mathcal{A}^{(M)} \sim \omega^{-\frac{1}{2}}$ near $\omega=0$. Nevertheless, although the nature of the singularity seems more complicated than in the massless case, involving also Regge cuts, the Reggeon trajectory is still linear after the inclusion of terms of order $\mathcal{O}(m)$. Of course, this result is based on a certain number of approximations and assumptions; nevertheless, it shows how a non trivial Regge singularity can emerge from quark-mass effects.

\subsection{Prefactors}

Moreover, we show now that the linearity of the trajectory is a quite robust result, which does not change under the inclusion of the factor $2 \alpha_{\text {eff }}^{\prime} \chi$ removed "by hand" in eq. (7.3); more generally, we show that it does not change under the inclusion of possible extra factors of the form $s^{\delta \alpha} \chi^{n_{\chi}} b^{n_{b}}$, which could come from the string fluctuations and from the quadratic fluctuations around the saddle point. Clearly, a factor $s^{\delta \alpha}$ simply shifts the trajectory, changing the intercept of an amount $\delta \alpha$. According to the properties of the Mellin transform given in eq. (7.7), factors of the type $\chi^{n_{\chi}}$ (with $n_{\chi}>0$ ) require $n_{\chi}$ derivatives with respect to $\omega$, so increasing the order of poles, but leaving the linearity of the trajectory unaltered. Finally, factors of the type $b^{n_{b}}$ (with $n_{b}>0$ ) can be of two types, namely $n_{b}$ even or $n_{b}$ odd. Notice that the term $\mathcal{T}_{0}$ in eq. (7.4) comes from a twodimensional integral of the type Gaussian times an even power of $b$, namely $b^{0}$, while for the term $\mathcal{T}_{1}$ the integral is of the type Gaussian times an odd power of $b$, namely $b^{1}$. Let 
us indicate with $\mathcal{T}_{0,1}^{\left(n_{b}\right)}$ the modified integrals obtained including an extra $b^{n_{b}}$ factor in the integrand. In the case of $n_{b}$ even, $n_{b}=2 k$, the type of integral of $\mathcal{T}_{0,1}^{\left(n_{b}\right)}$ is the same as that of $\mathcal{T}_{0,1}$, and the extra factors are taken into account by performing $k$ derivatives as follows,

$$
\mathcal{T}_{0,1}^{(2 k)}=\left[4 \chi \alpha_{\text {eff }}^{\prime}\left(1+\alpha_{\text {eff }}^{\prime} \frac{\partial}{\partial \alpha_{\text {eff }}^{\prime}}\right)\right]^{k} \mathcal{T}_{0,1},
$$

which are again seen not to change the linear trajectory. For $n_{b}$ odd, $n_{b}=2 k+1, \mathcal{T}_{0}^{\left(n_{b}\right)}$ becomes of the type Gaussian times an odd power of $b$, and similarly $\mathcal{T}_{1}^{\left(n_{b}\right)}$ becomes of the type Gaussian times an even power of $b$. More precisely,

$$
\begin{aligned}
& \mathcal{T}_{0}^{\left(n_{b}\right)}=\mathcal{T}_{0}^{(2 k+1)}=\frac{\chi}{4 m} \mathcal{T}_{1}^{(2 k)}, \\
& \mathcal{T}_{1}^{\left(n_{b}\right)}=\mathcal{T}_{1}^{(2 k+1)}=\frac{4 m}{\chi} \mathcal{T}_{0}^{(2 k+2)},
\end{aligned}
$$

and the result above in eq. (7.19) for even $n_{b}$ can be applied. In conclusion, the linearity of the Regge trajectory is not affected by the class of modifications considered here; in particular, the slope of the trajectory does not change. ${ }^{32}$

\subsection{Multi-sheet structure of the effective action: convolution of Regge ampli- tudes}

The results discussed so far are based on the use of the " $-i \varepsilon$ " prescription for the analytic continuation of eq. (6.2) from $b<b_{c}$ to $b>b_{c}$, leading to eq. (6.5) for the Minkowskian effective action. As we have mentioned in the previous section, it is possible that the whole multi-sheet structure of the Minkowskian effective action is physically relevant. A careful analysis shows that in the most general case the analytic continuation of eq. (6.2) from $b<b_{c}$ to $b>b_{c}$ leads to

$$
\begin{aligned}
\left.S_{\text {eff, M }}\right|_{b<b_{c}} \rightarrow & \left.S_{\text {eff, M }}^{( \pm, n)}\right|_{b>b_{c}}= \\
& \pm\left\{\frac{b^{2}}{2 \pi \alpha_{\text {eff }}^{\prime} \chi} \arccos \frac{b_{c}}{b}-\frac{2 b m}{\chi} \sqrt{1-\left(\frac{b_{c}}{b}\right)^{2}}\right\}+2 \pi^{2} \alpha_{\text {eff }}^{\prime} m^{2}+\frac{n b^{2}}{\alpha_{\text {eff }}^{\prime} \chi},
\end{aligned}
$$

with $n \in \mathbb{Z}$, depending on the specific prescription chosen for the analytic continuation, i.e., on the path in the complex plane along which the analytic continuation is performed. Here $\arccos x$ denotes the principal determination of the inverse cosine function, i.e., $\arccos x \in$ $[0, \pi]$. The last term in eq. (7.21) comes from the analytic continuation of this function, $\arccos x \rightarrow \pm \arccos x+2 n \pi i$, along paths in the complex plane which wind a certain number of times around -1 .

As we have already said, we do not have a precise mathematical argument which would select a specific prescription, and so one of the possibilities $\left.S_{\mathrm{eff}, \mathrm{M}}^{( \pm, n)}\right|_{b>b_{c}}$ for the Minkowskian

\footnotetext{
${ }^{32}$ It can be shown that the term neglected in eq. (3.19) would not change the Reggeon trajectory. At the order $\mathcal{O}(m)$ considered here, its effect could be taken into account by replacing $m \rightarrow m-\delta c$ in the formulas of this section.
} 
effective action. As a consequence, we have to use physical arguments in order to discriminate among the various possibilities. A first requirement, related to the unitarity bound on the impact-parameter amplitude, is that the resulting amplitude vanishes for $b \rightarrow \infty$.

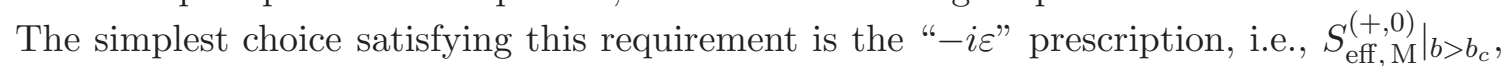
but it is clearly not the only one. A second reasonable requirement is that the last term in eq. (7.21) may be interpreted as a correction to a given basic amplitude for Reggeon exchange. Stated differently, we ask that setting $n=0$ we obtain a physically acceptable quantity. These two requirements restrict the possibilities to $\left.S_{\text {eff, M }}^{(+, n)}\right|_{b>b_{c}}$ with $n \in \mathbb{N}$.

We will make now the following working hypothesis: we will assume that all the physically sensible choices $\left.S_{\text {eff, }}^{(+, n)}\right|_{b>b_{c}}, n \in \mathbb{N}$, contribute to the Reggeon-exchange amplitude. The determination of the full contribution of each of the admissible terms to the scattering amplitude appears to be a difficult task, which would require the knowledge of their relative weights in the functional integral eq. (3.3) (after analytic continuation to Minkowski space-time). However, from their analytical structure and formal properties, the new contributions can be put into a relation with physical processes which are expected to take place in meson-meson scattering at high energy.

Indeed, one finds that each contribution to the impact-parameter amplitude is proportional to the following factorised expression,

$$
\exp \left\{-S_{\mathrm{eff}, \mathrm{M}}^{(+, n)}\right\}=\exp \left\{-S_{\mathrm{eff}, \mathrm{M}}\right\} \times\left[\exp \left\{-\frac{b^{2}}{\alpha_{\mathrm{eff}}^{\prime} \chi}\right\}\right]^{n}
$$

where $S_{\text {eff, M }} \equiv S_{\text {eff, M }}^{(+, 0)}$ is the effective action given explicitly in eq. (6.5), corresponding to the Reggeon-exchange amplitude discussed in the previous section, and where for notational simplicity we have dropped the subscript $\left.\right|_{b>b_{c}}$. Going from impact-parameter to transverse momentum space via Fourier transform, and ignoring possible $b$-dependent prefactors, which can be treated as discussed in the previous subsection, one obtains for each component

$$
\begin{aligned}
\mathcal{A}^{(+, n)}\left(s, t=-\vec{q}^{2}\right) & \equiv \int d^{2} b e^{i \vec{q} \cdot \vec{b}} \exp \left\{-S_{\text {eff, M }}^{(+, n)}\right\} \\
& =\int d^{2} b e^{i \vec{q} \cdot \vec{b}} \exp \left\{-S_{\text {eff, }, \mathrm{M}}\right\}\left[\exp \left\{-\frac{b^{2}}{\alpha_{\text {eff }}^{\prime} \chi}\right\}\right]^{n} \\
& =i^{n} \mathcal{A}^{(+, 0)}(s, t) \otimes \mathcal{A}_{\text {el }}^{\otimes n}(s, t),
\end{aligned}
$$

where $\otimes$ is the sign of a convolution, defined here as

$$
f(t) \otimes g(t) \equiv \frac{1}{2 s} \int \frac{d^{2} k}{(2 \pi)^{2}} f\left(-(\vec{q}-\vec{k})^{2}\right) g\left(-\vec{k}^{2}\right), \quad t=-\vec{q}^{2},
$$

and where the amplitudes $\mathcal{A}^{(+, 0)}$ and $\mathcal{A}_{e l}$ are given by

$$
\begin{aligned}
\mathcal{A}^{(+, 0)}(s, t) & =\int d^{2} b e^{i \vec{q} \cdot \vec{b}} \exp \left\{-S_{\text {eff, } \mathrm{M}}\right\}, \\
\mathcal{A}_{e l}(s, t) & =-i 2 s \int d^{2} b e^{i \vec{q} \cdot \vec{b}} \exp \left\{-\frac{b^{2}}{\alpha_{\text {eff }}^{\prime} \chi}\right\}=-2 i \pi s \alpha_{\text {eff }}^{\prime} \chi e^{\frac{\alpha_{\text {eff }}^{\prime}}{4} \chi} .
\end{aligned}
$$


The physical interpretation of the resulting convolution (7.23) becomes quite clear when remarking that the amplitude $\mathcal{A}_{e l}(s, t)$ given by eq. (7.25) is equal (up to prefactors) to the one obtained for elastic dipole-dipole scattering within the same formalism in ref. [12]. This means that the various components $\mathcal{A}^{(+, n)}(s, t)$ represent the contribution of multiple elastic rescattering interaction between the colliding mesons, occuring together with the $q \bar{q}$-Reggeon exchange previously discussed, which corresponds to the amplitude $\mathcal{A}^{(+, 0)}(s, t)$. We find that such elastic contributions are independent of the quark mass, as it is expected, and moreover of Regge-pole type, with Regge trajectory $\alpha_{e l}(t)=\alpha_{0 e l}+\alpha_{e l}^{\prime} t$. As already noticed in [14], the "Regge slope" $\alpha_{e l}^{\prime}=\alpha_{\text {eff }}^{\prime} / 4$ of the elastic amplitude (7.25) is one-fourth of the one obtained in the case of $q-\bar{q}$ exchange, and the "Regge intercept" is $\alpha_{0 e l}=1$ (up to fluctuations, see [13]).

From a phenomenological point of view, such contributions are expected to come from the long interaction time allowed by the softness of the interactions at strong coupling in QCD (although a complete theoretical derivation is not yet available). We see here that they may appear in the gauge/gravity framework in relation with the multi-sheet structure of the effective action, if one assumes that all the sheets which are physically sensible (in the sense discussed above) contribute to the scattering amplitude. Although a satisfactory mathematical justification of this assumption is lacking at the moment, a possible origin of these extra contributions is the following. When formulated in terms of the variable $\varphi(s)$, the Euclidean variational problem is invariant under the reparameterisation $\varphi(s) \rightarrow \varphi(s)+2 n \pi i$. On the other hand, the expression eq. (5.21) for the Euclidean effective action is not: while it is obviously possible to write it in an explicit reparameterisationinvariant form, in doing so one would lose analyticity in $\tilde{\varphi}$. Since an analytic expression is required in order to go from Euclidean to Minkowski space, one has to impose a "gauge choice" (e.g., $\operatorname{Im} \varphi(s)=0$ ), and use the corresponding expression for the Euclidean effective action (which in this case would be eq. (5.21)). As a result, it is possible that the completely equivalent choices $\varphi(s)+2 n \pi i$ in Euclidean space are mapped into different solutions of the corresponding variational problem in Minkowski space, each one contributing to the path integral a quantity proportional to expression eq. (7.22). This possibility is currently under investigation.

\section{Conclusions and outlook}

In this paper we have investigated the problem of $q \bar{q}$-Reggeon exchange in soft high-energy meson-meson scattering, in the framework of the gauge/gravity duality in a generic confining background, along the lines of [14]. Reggeon exchange is described as quark-antiquark exchange in the $t$ channel between the two mesons, represented as wave packets of colourless quark-antiquark dipoles. After Wick rotation to Euclidean space, the corresponding impact-parameter amplitude is represented in terms of a path-integral of Wilson loops, running along the trajectories of the constituent partons. While the trajectories of the "spectator" quark and antiquark can be dealt with in an eikonal approximation, the trajectories of the exchanged fermions have to be integrated over, with a weight depending on their length. In the dual gravity picture, where a Wilson loop corresponds to a minimal 
surface having the loop contour as boundary, the exchanged-fermion trajectories become therefore what we have called floating boundaries, which should in principle be integrated over. In the case of heavy mesons, corresponding to small dipole sizes, the floating boundaries which give the dominant contribution to the Euclidean path-integral are expected to lie on a helicoid, determined by the eikonal trajectories of the partons, and they can be determined in a saddle-point approximation by solving what we have called a "minimal surface problem with floating boundary", involving both the area of the surface and the length of the boundary in the minimisation procedure. The properties of the Reggeon trajectory are therefore related to the properties of the solution of this problem, which we have investigated in detail. Including the effects of a small but non-zero constituent quark mass $m$, we have found a real solution to such an equation, in a limited interval of values of the impact-parameter $b \leq b_{c} \propto m$. After analytic continuation into Minkowski space-time, and a subsequent analytic continuation in $b$ to extend the result to the region $b>b_{c}$, we have derived an expression for the amplitude in the case of non-zero quark mass, which reduces to the result for massless quarks discussed in [14] in the limit $m \rightarrow 0$.

The advantage of keeping the quark mass different from zero is twofold: on one side, it regularises the calculation, allowing a rigorous analysis of the solution of the saddlepoint equation in Euclidean space, and of the physical amplitude obtained after analytic continuation. On the other side, it allows to compute mass-dependent corrections to the amplitude, and to investigate the modifications of the Reggeon singularity due to the quark mass. To first order in $m$, it turns out that the Reggeon singularity is more complicated than a Regge pole, but that nevertheless the Reggeon trajectory is the same found in the massless case, namely $\alpha_{\mathcal{R}}(t)=\alpha_{\text {eff }}^{\prime} t$, if we neglect string fluctuations around the minimal surface, and quadratic fluctuations of the boundary around the saddle-point solution. We have discussed a quite large class of possible modifications of the amplitude due to these effects, and we have shown that while the nature of the singularity can change, the linearity of the Reggeon trajectory is not affected; in particular, the slope of the trajectory does not change.

Let us now discuss the outlook on possible future directions of investigation. As discussed in section 6, in order to perform correctly the analytic continuation from Euclidean to Minkowski space-time one should know the exact dependence on the Euclidean angle $\theta$. Although we have been able to write down the exact solution to the saddle-point equation, nevertheless we could not obtain it in a sufficiently explicit form, displaying the exact dependence on the relevant variables. Our conclusions regarding the Reggeon trajectory rely on an approximate explicit expression, analytically continued to Minkowski space-time, and require therefore further investigation to be consolidated. However, the qualitative agreement with the phenomenology let us hope that more precise calculations of the $\theta$-dependence would not change too much the result.

In the appendix we have computed the contribution of the spin factor in Euclidean space in exact implicit form, and in an approximate explicit form suitable for the analytic continuation to Minkowski space-time. However, we are unable for the moment to perform reliably the analytic continuation to $b>b_{c}$, and so we have preferred not to include the spin factor in our analysis. Although spin effects are not expected to change the Reggeon trajectory, a detailed study is needed to clarify this issue. 
The corrections due to string fluctuations have been computed in [14] in the massless case, where they have been shown to give a contribution $\delta \alpha_{0}=n_{\perp} / 24$ to the Reggeon intercept, but we have not performed the corresponding computation in the massive case considered in this paper. Moreover, at the present stage the effect of fluctuations around the saddle-point solution are not known. This point deserves further investigation.

Another open issue is that of the origin of the companion contributions, discussed in section 7 . These contributions, identified with the effect of rescattering interactions between the colliding mesons, have been obtained from the multi-sheet structure of the Minkowskian effective action, and it has been suggested that they are due to the non-uniqueness of the solution of the variational problem when formulated in Minkowski space. A detailed investigation of this problem is needed in order to better substantiate this suggestion.

As we have already remarked in section 3, the basic formula for the Reggeon-exchange amplitude has been suggested in [14], rather than having been directly derived from QCD first principles. Such a derivation is in progress, and it seems to confirm essentially the expression used in [14] and in this paper: a detailed report will be published in a forthcoming paper [69].

Another interesting issue is that of corrections related to the inclusion of dynamicalfermion effects, which are subleading in a $1 / N_{c}$ expansion but which could be relevant for the dependence on energy of the Reggeon-exchange amplitude. Using a path-integral representation for the fermion-matrix determinant, such corrections can be computed with the same minimal-surface formalism employed here (see for example [70]). This computation is in progress, and will be discussed in a separate publication [71].

In conclusion, we hope that the renewed interest in the study of soft high energy scattering in the modern framework of gauge/gravity duality will lead to a better understanding of the old but nevertheless still open problem of Regge amplitudes.

\section{Acknowledgments}

We acknowledge useful discussions with R. Janik and G. Korchemsky. M.G. wants to thank the Institut de Physique Théorique, Saclay, where part of this work has been done, for the kind hospitality. This work has been partly funded by a grant of the "Fondazione Angelo Della Riccia" (Firenze, Italy). M.G. is supported by MICINN under the CPAN project CSD2007-00042 from the Consolider-Ingenio2010 programm, as well as under the grant FPA2009-09638.

\section{A Evaluation of the spin factor}

In this appendix we critically repeat the calculation of [14] for the spin factor $I_{D}[\dot{x}(\nu)][48-50]$ in $D=4$ Euclidean space, for the special case of a path contained in a $3 D$ hyperplane. Here $\dot{x}(\nu)$ is the derivative of the path $x(\nu)$ with respect to the natural 
parameter $\nu$, so that $(\dot{x}(\nu))^{2}=1$. The spin factor is defined as

$$
\begin{aligned}
I_{D}[\dot{x}(\nu)] & =\lim _{N \rightarrow \infty} \prod_{k=1}^{N} \frac{1+\not k(k \tau)}{2} \\
\tau & =\frac{L}{N}
\end{aligned}
$$

where $L$ is the length of the path, so that $\nu \in[0, L]$. We have denoted $\not h=n_{\mu} \gamma_{E \mu}$ with $\gamma_{E \mu}$ the Euclidean Dirac matrices, satisfying the Euclidean Clifford algebra $\left\{\gamma_{E \mu}, \gamma_{E \nu}\right\}=2 \delta_{\mu \nu}$, which in four dimensions read

$$
\gamma_{E 4}=\gamma^{0}=\left(\begin{array}{cc}
\mathbb{1}_{2} & 0 \\
0 & -\mathbb{1}_{2}
\end{array}\right), \quad \gamma_{E j}=-i \gamma^{j}=\left(\begin{array}{cc}
0 & -i \sigma^{j} \\
i \sigma^{j} & 0
\end{array}\right),
$$

where $\mathbb{1}_{D}$ is the $D$-dimensional identity matrix, $\gamma^{\mu}$ are the Minkowskian gamma-matrices, and $\sigma^{j}$ are the Pauli matrices

$$
\sigma^{1}=\left(\begin{array}{ll}
0 & 1 \\
1 & 0
\end{array}\right), \quad \sigma^{2}=\left(\begin{array}{cc}
0 & -i \\
i & 0
\end{array}\right), \quad \sigma^{3}=\left(\begin{array}{ll}
1 & 0 \\
0 & 1
\end{array}\right) .
$$

It is immediate to see that for $n^{2}=1$ the quantity

$$
P(n)=\frac{1+\not h}{2}
$$

is a projector, i.e., $[P(n)]^{2}=[P(n)]^{\dagger}=P(n)$. Let us consider now the case of interest, namely $D=4$

$$
\dot{x}(\nu)=\left(\dot{x}_{4}, \dot{x}_{1}, \dot{x}_{2}, \dot{x}_{3}\right), \quad \dot{x}_{4}^{2}+\dot{x}_{1}^{2}+\dot{x}_{2}^{2}+\dot{x}_{3}^{2}=1,
$$

and a path contained in the $3 D$ hyperplane $x_{3}=$ const., i.e., $\dot{x}_{3}=0$. The projector (A.4) has therefore the form

$$
P(\dot{x}(\nu))=\frac{1}{2}\left(\begin{array}{cccc}
1+\dot{x}_{4} & 0 & 0 & -i \dot{x}_{1}-\dot{x}_{2} \\
0 & 1+\dot{x}_{4} & -i \dot{x}_{1}+\dot{x}_{2} & 0 \\
0 & i \dot{x}_{1}+\dot{x}_{2} & 1-\dot{x}_{4} & 0 \\
i \dot{x}_{1}-\dot{x}_{2} & 0 & 0 & 1-\dot{x}_{4}
\end{array}\right),
$$

which is easily recognised as the direct sum of two two-dimensional projectors. To see this explicitly, the matrix $P(\dot{x})$ can be brought to block-diagonal form,

$$
\begin{array}{rlrl}
P(\dot{x}(\nu)) & =M^{T} \bar{P}(\dot{x}(\nu)) M, & \bar{P}(\dot{x}(\nu)) & =\left(\begin{array}{c|c}
\bar{P}_{1} & 0 \\
\hline 0 & \bar{P}_{2}
\end{array}\right), \\
\bar{P}_{1}(\dot{x}(\nu))=\frac{1}{2}\left(\begin{array}{cc}
1+\dot{x}_{4} & -i \dot{x}_{1}-\dot{x}_{2} \\
i \dot{x}_{1}-\dot{x}_{2} & 1-\dot{x}_{4}
\end{array}\right), & \bar{P}_{2}(\dot{x}(\nu))=\frac{1}{2}\left(\begin{array}{cc}
1+\dot{x}_{4} & -i \dot{x}_{1}+\dot{x}_{2} \\
i \dot{x}_{1}+\dot{x}_{2} & 1-\dot{x}_{4}
\end{array}\right),
\end{array}
$$

where the matrix $M$ is given by

$$
M_{i j}=\left\{\begin{array}{ll}
1 & \text { for }(i, j)=(1,1),(2,4),(3,2),(4,3), \\
0 & \text { otherwise }
\end{array} \quad M^{T} M=\mathbb{1}_{4}\right.
$$


and one can easily verify that $\bar{P}_{1,2}^{2}=\bar{P}_{1,2}^{\dagger}=\bar{P}_{1,2}$. Moreover, ${ }^{33}$

$$
\begin{array}{lll}
\bar{P}_{1}=\frac{1+\vec{u}_{1} \cdot \vec{\sigma}}{2}=\bar{P}_{1}\left(\vec{u}_{1}(\nu)\right), & \vec{u}_{1}(\nu)=\left(-\dot{x}_{2}, \dot{x}_{1}, \dot{x}_{4}\right), & \vec{u}_{1}^{2}=1, \\
\bar{P}_{2}=\frac{1+\vec{u}_{2} \cdot \vec{\sigma}}{2}=\bar{P}_{2}\left(\vec{u}_{2}(\nu)\right), & \vec{u}_{2}(\nu)=\left(\dot{x}_{2}, \dot{x}_{1}, \dot{x}_{4}\right), & \vec{u}_{2}^{2}=1,
\end{array}
$$

and since in three dimensions the gamma-matrices are equal to the Pauli matrices, $\bar{P}_{1,2}$ are exactly the projectors entering the definition of the three-dimensional spin factor. One can thus write

$$
I_{4}[\dot{x}]=M^{T}\left(\begin{array}{c|c}
I_{3}\left[\vec{u}_{1}\right] & 0 \\
\hline 0 & I_{3}\left[\vec{u}_{2}\right]
\end{array}\right) M,
$$

and exploit the explicit expression for the three-dimensional spin factor [77],

$$
I_{3}\left[\vec{u}_{j}\right]=\frac{1+\vec{u}_{j}(L) \cdot \vec{\sigma}}{2} e^{-\frac{i}{2} \Phi\left(\mathcal{C}_{\vec{u}_{j}}\right)} \frac{1+\vec{u}_{j}(0) \cdot \vec{\sigma}}{2}\left(\frac{1+\vec{u}_{j}(L) \cdot \vec{u}_{j}(0)}{2}\right)^{-\frac{1}{2}},
$$

where $\Phi\left(\mathcal{C}_{\vec{u}_{j}}\right)$ is the area of the portion of sphere delimited by the closed path $\mathcal{C}_{\vec{u}_{j}}$ made up of the path $\vec{u}_{j}(\nu)$ and by the segment of great circle connecting the points $\vec{u}_{j}(L)$ and $\vec{u}_{j}(0)$ (see figure 14). Explicitly,

$$
\begin{aligned}
\Phi\left(\mathcal{C}_{\vec{u}_{j}}\right) & =\oint_{\mathcal{C}_{\vec{u}_{j}}} d t \dot{\phi}(1-\cos \omega), \\
\vec{u}_{j}(\nu) & =(\sin \omega \cos \phi, \sin \omega \sin \phi, \cos \omega) .
\end{aligned}
$$

It is immediate to see that this quantity changes sign under inversion of the orientation of the path; moreover, it is invariant under rotations of the path, and it changes sign under parity. ${ }^{34}$ As a consequence, since $\vec{u}_{2}=\mathcal{P} \mathcal{R} \vec{u}_{1}$, where $\mathcal{P}$ is parity and $\mathcal{R}$ an appropriate rotation, and $\vec{u}_{1}=\mathcal{R}^{\prime} \vec{u}$ with

$$
\vec{u}=\left(\dot{x}_{4}, \dot{x}_{1}, \dot{x}_{2}\right)
$$

for an appropriately chosen rotation $\mathcal{R}^{\prime}$, we can write

$$
\Phi\left(\mathcal{C}_{\vec{u}_{1}}\right)=\Phi\left(\mathcal{C}_{\vec{u}}\right), \quad \Phi\left(\mathcal{C}_{\vec{u}_{2}}\right)=-\Phi\left(\mathcal{C}_{\vec{u}_{1}}\right)=-\Phi\left(\mathcal{C}_{\vec{u}}\right)
$$

This form will be useful when applying the general expression to our specific case in the Reggeon-exchange calculation. Finally, noting that $\vec{u}_{1}(L) \cdot \vec{u}_{1}(0)=\vec{u}_{2}(L) \cdot \vec{u}_{2}(0)=\dot{x}(L)$. $\dot{x}(0)$, and setting

$$
\overline{\mathcal{U}}=\left(\begin{array}{c|c}
e^{-\frac{i}{2} \Phi\left(\mathcal{C}_{\vec{u}}\right)} & 0 \\
\hline 0 & e^{\frac{2}{2} \Phi\left(\mathcal{C}_{\vec{u}}\right)}
\end{array}\right), \quad \mathcal{N}=\left(\frac{1+\dot{x}(L) \cdot \dot{x}(0)}{2}\right)^{-\frac{1}{2}},
$$

we can write the final expression

$$
I_{4}[\dot{x}]=\mathcal{N} P(\dot{x}(L)) \mathcal{U} P(\dot{x}(0)),
$$

\footnotetext{
${ }^{33}$ Here and in the rest of the appendices, we denote with $\vec{v}$ a three-dimensional vector, while twodimensional vectors are denoted as $\vec{v}_{\perp}$.

${ }^{34}$ Possible extra contributions coming from a non-trivial winding of the path around the sphere are proportional to $4 \pi$, and thus irrelevant in the phase factor.
} 
where

$$
\mathcal{U}=M^{T} \overline{\mathcal{U}} M=\operatorname{diag}\left(e^{-\frac{i}{2} \Phi\left(\mathcal{C}_{\vec{u}}\right)}, e^{\frac{i}{2} \Phi\left(\mathcal{C}_{\vec{u}}\right)}, e^{\frac{i}{2} \Phi\left(\mathcal{C}_{\vec{u}}\right)}, e^{-\frac{i}{2} \Phi\left(\mathcal{C}_{\vec{u}}\right)}\right)
$$

is a diagonal matrix which commutes with the four-dimensional projectors $P(\dot{x}(L))$ and $P(\dot{x}(0))$, and which is easily seen to induce opposite rotations on the two two-spinor components of a Dirac four-spinor. Defining

$$
\Sigma^{3} \equiv\left(\begin{array}{c|c}
\sigma^{3} & 0 \\
\hline 0 & \sigma^{3}
\end{array}\right)
$$

we can write $\mathcal{U}$ as

$$
\mathcal{U}=\cos \left(\frac{\Phi\left(\mathcal{C}_{\vec{u}}\right)}{2}\right) \mathbb{1}_{4}-i \sin \left(\frac{\Phi\left(\mathcal{C}_{\vec{u}}\right)}{2}\right) \gamma^{0} \Sigma^{3}
$$

where for future utility we have made use of the Minkowskian gamma-matrix $\gamma^{0}$.

\section{B Application to the Reggeon-exchange amplitude}

We apply now the results of appendix A to the case of the Reggeon-exchange amplitude. We begin with the contraction of the spin factor, after analytic continuation to Minkowski space, with the bispinors associated to the scattering quarks and antiquarks. In the following subsection we will evaluate the phase factors corresponding to the relevant saddle-point solution.

\section{B.1 Contraction with the bispinors}

We are interested in the two following quantities,

$$
\begin{aligned}
& \mathcal{Q}_{+}=\left.\bar{u}^{\left(s_{q}^{\prime}\right)}\left(p_{q}^{\prime}\right) I_{4}\left[\dot{X}_{+}\right]\right|_{\theta \rightarrow-i \chi} v^{\left(t_{\bar{q}}^{\prime}\right)}\left(p_{\bar{q}}^{\prime}\right), \\
& \mathcal{Q}_{-}=\left.\bar{v}^{\left(t_{\bar{q}}\right)}\left(p_{\bar{q}}\right) I_{4}\left[\dot{X}_{-}\right]\right|_{\theta \rightarrow-i \chi} u^{\left(s_{q}\right)}\left(p_{q}\right),
\end{aligned}
$$

where $X_{ \pm}$are given in eq. (3.6), and, taking into account the softness of the process, ${ }^{35}$

$$
\begin{array}{ll}
p_{\bar{q}}^{\prime} \simeq p_{\bar{q}} \simeq\left(E, p, \overrightarrow{0}_{\perp}\right)=m \tilde{u}_{1}, & p_{q}^{\prime} \simeq p_{q}=\left(E,-p, \overrightarrow{0}_{\perp}\right)=m \tilde{u}_{2}, \\
\tilde{u}_{1}=\left(\cosh \frac{\chi}{2}, \sinh \frac{\chi}{2}, \overrightarrow{0}_{\perp}\right), & \tilde{u}_{2}=\left(\cosh \frac{\chi}{2},-\sinh \frac{\chi}{2}, \overrightarrow{0}_{\perp}\right),
\end{array}
$$

with $m$ the mass of the light quarks. Since we expect that the relevant paths deviate from the eikonal trajectory only near the interaction region, we have that

$$
\begin{aligned}
\dot{X}_{+}(0) & =-u_{1}, & \dot{X}_{+}(L) & =u_{2}, \\
\dot{X}_{-}(0) & =u_{2}, & \dot{X}_{-}(L) & =-u_{1}, \\
u_{1} & =\left(\cos \frac{\theta}{2}, \sin \frac{\theta}{2}, \overrightarrow{0}_{\perp}\right), & u_{2} & =\left(\cos \frac{\theta}{2},-\sin \frac{\theta}{2}, \overrightarrow{0}_{\perp}\right),
\end{aligned}
$$

\footnotetext{
${ }^{35}$ In a more rigorous treatment of meson-meson scattering, the mass of the quark $m$ in eq. (B.2) should be substituted with the meson-mass fraction carried by the constituent quarks [69].
} 
and moreover

$$
\mathcal{N}=\left(\frac{1-u_{1} \cdot u_{2}}{2}\right)^{-\frac{1}{2}}=\left(\frac{1-\cos \theta}{2}\right)^{-\frac{1}{2}} .
$$

Since the straight-line parts of the paths do not contribute to the phase factors, as we will show below, the spin factor should be independent of $T$, and therefore only the analytic continuation in the angular variable has to be performed. This is actually the case for the solution of the saddle-point equation. Performing now the analytic continuation, we obtain

$$
\begin{aligned}
& \dot{X}_{+}(0)=-\not \mu_{1} \rightarrow-\not \varkappa_{1}=-\frac{\not \phi_{\bar{q}}{ }^{\prime}}{m}, \quad \dot{X}_{+}(L)=\not_{2} \rightarrow \not{u}_{2}=\frac{\not p_{q}{ }^{\prime}}{m},
\end{aligned}
$$

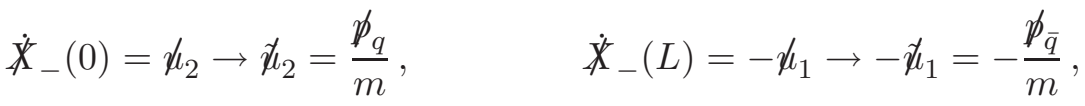

where it is understood that $\not{\iota}_{j}=\tilde{u}_{j \mu} \gamma^{\mu}$. In the high-energy, low momentum transfer limit we are interested in, the bispinors can be approximated as

$$
\begin{aligned}
& u^{(s)}(p)=\sqrt{E+m}\left(\begin{array}{c}
\phi^{(s)} \\
\frac{\vec{p} \cdot \vec{\sigma}}{E+m} \phi^{(s)}
\end{array}\right) \rightarrow \sqrt{E+m}\left(\begin{array}{c}
\phi^{(s)} \\
\frac{p \sigma^{1}}{E+m} \phi^{(s)}
\end{array}\right), \\
& v^{(t)}(p)=\sqrt{E+m}\left(\begin{array}{c}
\frac{\vec{p} \cdot \vec{\sigma}}{E+m} \tilde{\phi}^{(t)} \\
\tilde{\phi}^{(t)}
\end{array}\right) \rightarrow \sqrt{E+m}\left(\begin{array}{c}
\frac{p \sigma^{1}}{E+m} \tilde{\phi}^{(t)} \\
\tilde{\phi}^{(t)}
\end{array}\right),
\end{aligned}
$$

where $\phi^{(s)}$ and $\tilde{\phi}^{(t)}$ are two-component spinors. As a consequence, the bispinors are eigenstates of the projectors acting on them, so that

$$
\begin{aligned}
& \mathcal{Q}_{+}=\tilde{\mathcal{N}} \bar{u}^{\left(s_{q}^{\prime}\right)}\left(p_{q}^{\prime}\right) \tilde{\mathcal{U}}_{+} v^{\left(t_{\bar{q}}^{\prime}\right)}\left(p_{\bar{q}}^{\prime}\right), \\
& \mathcal{Q}_{-}=\tilde{\mathcal{N}} \bar{v}^{\left(t_{\bar{q}}\right)}\left(p_{\bar{q}}\right) \tilde{\mathcal{U}}_{-} u^{\left(s_{q}\right)}\left(p_{q}\right),
\end{aligned}
$$

where

$$
\tilde{\mathcal{N}}=\left.\mathcal{N}\right|_{\theta \rightarrow-i \chi}=\left(\frac{1-\cosh \chi}{2}\right)^{-\frac{1}{2}} \underset{\chi \rightarrow \infty}{\simeq}\left(1-\frac{s}{4 m_{1} m_{2}}\right)^{-\frac{1}{2}}
$$

and

$$
\begin{aligned}
\tilde{\mathcal{U}}_{ \pm} & =\operatorname{diag}\left(e^{-\frac{i}{2} \Phi^{(M)}\left(\mathcal{C}_{\vec{u}_{ \pm}}\right)}, e^{\frac{i}{2} \Phi^{(M)}\left(\mathcal{C}_{\vec{u}_{ \pm}}\right)}, e^{\frac{i}{2} \Phi^{(M)}\left(\mathcal{C}_{\vec{u}_{ \pm}}\right)}, e^{-\frac{i}{2} \Phi^{(M)}\left(\mathcal{C}_{\vec{u}_{ \pm}}\right)}\right), \\
\Phi^{(M)}\left(\mathcal{C}_{\vec{u}_{ \pm}}\right) & =\left.\Phi\left(\mathcal{C}_{\vec{u}_{ \pm}}\right)\right|_{\theta \rightarrow-i \chi} .
\end{aligned}
$$

It is now straightforward to evaluate these quantities, obtaining

$$
\begin{aligned}
& \mathcal{Q}_{+}=\tilde{\mathcal{N}} 2 p \phi^{\left(s_{q}^{\prime}\right) \dagger}\left(e^{\frac{i}{2} \Phi^{(M)}\left(\mathcal{C}_{\vec{u}_{+}}\right)} \sigma_{-}+e^{-\frac{i}{2} \Phi^{(M)}\left(\mathcal{C}_{\vec{u}_{+}}\right)} \sigma_{+}\right) \tilde{\phi}^{\left(t_{\bar{q}}^{\prime}\right)} \\
& \mathcal{Q}_{-}=\tilde{\mathcal{N}} 2 p \tilde{\phi}^{\left(t_{\bar{q}}\right) \dagger}\left(e^{\frac{i}{2} \Phi^{(M)}\left(\mathcal{C}_{\vec{u}_{-}}\right)} \sigma_{+}+e^{-\frac{i}{2} \Phi^{(M)}\left(\mathcal{C}_{\vec{u}_{-}}\right)} \sigma_{-}\right) \phi^{\left(s_{q}\right)}
\end{aligned}
$$

where $\sigma_{ \pm}$are the usual raising and lowering operators,

$$
\sigma_{ \pm}=\frac{\sigma^{1} \pm i \sigma^{2}}{2}
$$


The spinor base which gives the simplest representation is that of the eigenvectors of $\sigma^{3}$,

$$
\begin{array}{rlrl}
\sigma^{3} \phi^{(s)} & =s \phi^{(s)}, s= \pm 1, & \tilde{\phi}^{(t)} & =\phi^{(-t)}, t= \pm 1, \\
\phi^{(1)} & =\left(\begin{array}{l}
1 \\
0
\end{array}\right), & \phi^{(-1)}=\left(\begin{array}{l}
0 \\
1
\end{array}\right),
\end{array}
$$

and for this choice

$$
\begin{array}{ll}
\phi^{\left(s_{q}^{\prime}\right) \dagger} \sigma_{+} \tilde{\phi}^{\left(t_{\bar{q}}^{\prime}\right)}=\delta_{s_{q}^{\prime}, 1} \delta_{t_{\bar{q}}^{\prime}, 1}, & \phi^{\left(s_{q}^{\prime}\right) \dagger} \sigma_{-} \tilde{\phi}^{\left(t_{\bar{q}}^{\prime}\right)}=\delta_{s_{q}^{\prime},-1} \delta_{t_{\bar{q}}^{\prime},-1}, \\
\tilde{\phi}^{\left(t_{\bar{q}}\right) \dagger} \sigma_{-} \phi^{\left(s_{q}\right)}=\delta_{s_{q}, 1} \delta_{t_{\bar{q}}, 1}, & \tilde{\phi}^{\left(t_{\bar{q}}\right) \dagger} \sigma_{+} \phi^{\left(s_{q}\right)}=\delta_{s_{q},-1} \delta_{t_{\bar{q}},-1} .
\end{array}
$$

The evaluation of the other two terms contributing to the complete spin factor is trivial: since in that case $\dot{x}=u_{1,2}$ is constant along the trajectory, the path $\mathcal{C}_{\vec{u}}$ contracts to a point and the corresponding phase vanishes. Since the bispinors are again eigenstates of the projectors, one obtains simply $\left(p_{Q}^{\prime} \simeq p_{Q}, p_{\bar{Q}^{\prime}}^{\prime} \simeq p_{\bar{Q}^{\prime}}\right)$

$$
\begin{aligned}
& \mathcal{Q}_{1}=\bar{u}^{\left(s_{Q}^{\prime}\right)}\left(p_{Q}^{\prime}\right) I_{4}\left[\dot{X}_{1}\right] u^{\left(s_{Q}\right)}\left(p_{Q}\right)=\bar{u}^{\left(s_{Q}^{\prime}\right)}\left(p_{Q}\right) u^{\left(s_{Q}\right)}\left(p_{Q}\right)=2 m_{Q} \delta_{s_{Q}^{\prime}, s_{Q}}, \\
& \mathcal{Q}_{2}=-\bar{v}^{\left(t_{\bar{Q}^{\prime}}\right)}\left(p_{\bar{Q}^{\prime}}\right) I_{4}\left[\dot{X}_{2}\right] v^{\left(t_{\bar{Q}^{\prime}}^{\prime}\right)}\left(p_{\bar{Q}^{\prime}}^{\prime}\right)=-\bar{v}^{\left(t_{\bar{Q}^{\prime}}\right)}\left(p_{\bar{Q}^{\prime}}\right) v^{\left(t_{\bar{Q}^{\prime}}^{\prime}\right)}\left(p_{\bar{Q}^{\prime}}\right)=2 m_{\bar{Q}^{\prime}} \delta_{t_{\bar{Q}^{\prime}}, t_{\bar{Q}^{\prime}}^{\prime}},
\end{aligned}
$$

where $m_{Q}$ and $m_{\bar{Q}^{\prime}}$ are the masses of the heavy quark and antiquark, respectively. ${ }^{36}$

\section{B.2 Evaluation of the phase factor on the solution of the saddle-point equation}

We evaluate now the phase factor

$$
\begin{aligned}
\Phi\left(\mathcal{C}_{\vec{u}}\right) & =\oint_{\mathcal{C}_{\vec{u}}} d \nu \dot{\phi}(1-\cos \omega), \\
\vec{u} & =\left(\dot{x}_{4}, \dot{x}_{1}, \dot{x}_{2}\right)=(\sin \omega \cos \phi, \sin \omega \sin \phi, \cos \omega),
\end{aligned}
$$

for the solution of the saddle-point equation. The paths which we are interested in are given by

$$
x_{4}^{(+)}=\tau(-\sigma) \cos \left(-\frac{\theta \sigma}{b}\right), \quad x_{1}^{(+)}=\tau(-\sigma) \sin \left(-\frac{\theta \sigma}{b}\right), \quad x_{2}^{(+)}=-\sigma,
$$

and

$$
x_{4}^{(-)}=-\tau(\sigma) \cos \left(\frac{\theta \sigma}{b}\right), \quad x_{1}^{(-)}=-\tau(\sigma) \sin \left(\frac{\theta \sigma}{b}\right), \quad x_{2}^{(-)}=\sigma,
$$

with $\sigma \in[-b / 2, b / 2]$ in both cases, and $\tau(\sigma)$ being the solution of the saddle-point equation eq. (3.22). The minus signs are due to the orientation of the path, which is in principle relevant in this calculation, while it was not in the saddle-point equation. Although $\sigma$ is not the natural parameter, so that in the expression above it stands for $\sigma=\sigma(\nu)$, we find convenient for notational simplicity to not show explicitly its dependence on $\nu$. Notice that

\footnotetext{
${ }^{36}$ The minus sign in the contribution of the heavy antiquark compensates for an extra minus sign included in the eikonal approximation for the antiquark propagator.
} 
the symmetry of the path implies that $\sigma(L-\nu)=-\sigma(\nu)$, i.e., reversing the orientation is equivalent to flip the sign of $\sigma$. Moreover, since $\tau(\sigma)=\tau(-\sigma)$ we can write

$$
x_{4}^{(+)}=\tau(\sigma) \cos \left(\frac{\theta \sigma}{b}\right), \quad x_{1}^{(+)}=-\tau(\sigma) \sin \left(\frac{\theta \sigma}{b}\right), \quad x_{2}^{(+)}=-\sigma,
$$

and thus both paths are seen to be connected by a rotation to the path

$$
x_{4}=\tau(\sigma) \cos \left(\frac{\theta \sigma}{b}\right), \quad x_{1}=\tau(\sigma) \sin \left(\frac{\theta \sigma}{b}\right), \quad x_{2}=\sigma,
$$

and so are their derivatives with respect to the natural parameter. As a consequence the phases $\Phi\left(\mathcal{C}_{\vec{u}_{ \pm}}\right)$are both equal to the phase $\Phi\left(\mathcal{C}_{\vec{u}}\right)$ for the path $x,{ }^{37}$ so that the spin factor $\mathcal{Q} \equiv \mathcal{Q}_{+} \mathcal{Q}_{-} \mathcal{Q}_{1} \mathcal{Q}_{2}$ reduces to

$$
\begin{aligned}
\mathcal{Q}= & \mathcal{K} \delta_{s_{Q}^{\prime}, s_{Q}} \delta_{t_{\bar{Q}^{\prime}}, t_{\bar{Q}^{\prime}}^{\prime}} \delta_{s_{q} t_{\bar{q}}} \\
& \times\left[\delta_{s_{q} s_{q}^{\prime}} \delta_{t_{\bar{q}} t_{\bar{q}}}\left(e^{i \Phi^{(M)}\left(\mathcal{C}_{\vec{u}}\right)} \delta_{s_{q},-1}+e^{-i \Phi^{(M)}\left(\mathcal{C}_{\vec{u}}\right)} \delta_{s_{q}, 1}\right)+\delta_{s_{q},-s_{q}^{\prime}} \delta_{\bar{q}_{\bar{q}},-t_{\bar{q}}^{\prime}}\right], \\
\mathcal{K}= & 4(2 p \tilde{\mathcal{N}})^{2} m_{Q^{\prime}} m_{\bar{Q}^{\prime}} \underset{s \rightarrow \infty}{\simeq}-16 m_{1} m_{2} m_{Q} m_{\bar{Q}^{\prime}} .
\end{aligned}
$$

We turn now to the computation of $\Phi\left(\mathcal{C}_{\vec{u}}\right)$. Using the dimensionless variables $t(s)=p \tau(\sigma)$, $s=p \sigma$, with $p=\theta / b$, the derivatives $\dot{x}_{\mu}=d x_{\mu} / d \nu$ with respect to the natural parameter $\nu$ are written as

$$
\left\{\begin{array}{l}
\dot{x}_{4}=\frac{1}{\sqrt{1+t^{2}+t^{\prime 2}}}\left(t^{\prime} \cos s-t \sin s\right) \\
\dot{x}_{1}=\frac{1}{\sqrt{1+t^{2}+t^{\prime 2}}}\left(t^{\prime} \sin s+t \cos s\right) \\
\dot{x}_{2}=\frac{1}{\sqrt{1+t^{2}+t^{\prime 2}}}
\end{array}\right.
$$

where $s \in[-\theta / 2, \theta / 2]$ and the prime denotes derivative with respect to $s$, and

$$
\frac{d s}{d \nu}=\frac{\theta}{b} \frac{1}{\sqrt{1+t^{2}+t^{\prime 2}}}=\frac{\theta}{b} \dot{x}_{2} .
$$

Changing variables to $t=\sinh \varphi$ we have

$$
\left\{\begin{array}{l}
\dot{x}_{4}=\frac{1}{\cosh \varphi \sqrt{1+\varphi^{\prime 2}}}\left(\varphi^{\prime} \cosh \varphi \cos s-\sinh \varphi \sin s\right)=\dot{x}_{2} f_{4} \\
\dot{x}_{1}=\frac{1}{\cosh \varphi \sqrt{1+\varphi^{\prime 2}}}\left(\varphi^{\prime} \cosh \varphi \sin s+\sinh \varphi \cos s\right)=\dot{x}_{2} f_{1} \\
\dot{x}_{2}=\frac{1}{\cosh \varphi \sqrt{1+\varphi^{\prime 2}}} .
\end{array}\right.
$$

Recalling now the properties of the solution $\varphi(s)$,

$$
\lim _{s \rightarrow \pm \frac{\theta}{2}} \varphi^{\prime}(s)= \pm \infty, \quad \varphi(-s)=\varphi(s) \geq 0, \quad \varphi^{\prime}(s) \geq 0, \quad \varphi^{\prime}(0)=0,
$$

\footnotetext{
${ }^{37}$ This can be seen even more directly for $X_{+}$. By definition the path $X_{+}$is the path $x$ with its orientation reversed, $X_{+}(\nu)=x(L-\nu)$, so that $\dot{X}_{+}(\nu)=-\dot{x}(L-\nu)=\mathcal{P} \dot{x}(L-\nu)$. Since the phase $\Phi\left(\mathcal{C}_{\vec{u}}\right)$ changes sign under parity and when reversing the path, the desired equality follows.
} 


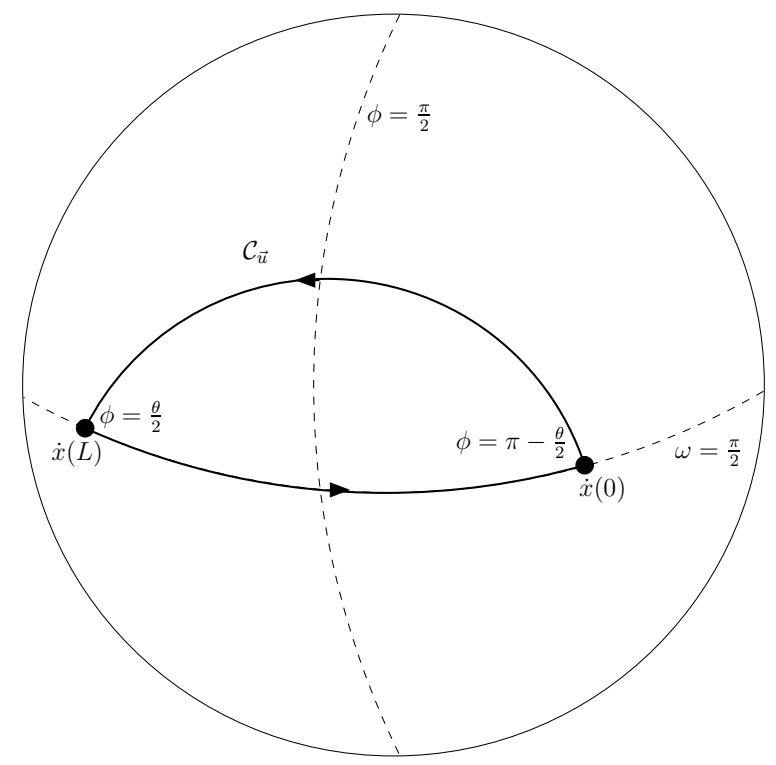

Figure 14. Phase factor for the saddle-point solution. Schematic representation of the path $\vec{u}(\nu)$, see eqs. (B.15) and (B.19). The phase $\Phi\left(\mathcal{C}_{\vec{u}}\right)$ is given by the area enclosed by the path.

one finds ${ }^{38}$

$$
\begin{aligned}
& \dot{x}_{4}\left( \pm \frac{\theta}{2}\right)= \pm \cos \frac{\theta}{2}, \quad \dot{x}_{1}\left( \pm \frac{\theta}{2}\right)=\sin \frac{\theta}{2}, \quad \dot{x}_{2}\left( \pm \frac{\theta}{2}\right)=0, \\
& \dot{x}_{4}(0)=0, \quad \dot{x}_{1}(0)=\tanh \varphi_{0}, \quad \dot{x}_{2}(0)=\frac{1}{\cosh \varphi_{0}},
\end{aligned}
$$

so that

$$
\begin{aligned}
& \omega\left(-\frac{\theta}{2}\right)=\frac{\pi}{2}, \quad \omega(0)=\arccos \frac{1}{\cosh \varphi_{0}}, \quad \omega\left(\frac{\theta}{2}\right)=\frac{\pi}{2}, \\
& \phi\left(-\frac{\theta}{2}\right)=\pi-\frac{\theta}{2}, \quad \phi(0)=\frac{\pi}{2}, \quad \phi\left(\frac{\theta}{2}\right)=\frac{\theta}{2} .
\end{aligned}
$$

One can prove that $\phi \neq 0, \pi, \omega \neq 0, \pi$, so that the path lies in the sector $0<\phi<\pi$, $0<\omega<\pi / 2$ of the sphere and so does not wind around it; also, the sign of $\dot{\omega}$ is the same as the sign of $\varphi^{\prime}$. A schematic representation of the path is given in figure 14. Note that the segment of great circle closing the path lies at $\omega=\pi / 2$. We have therefore

$$
\Phi\left(\mathcal{C}_{\vec{u}}\right)=\oint_{\mathcal{C}_{\vec{u}}} d \nu \dot{\phi}(1-\cos \omega)=-\int_{0}^{L} d \nu \dot{\phi} \cos \omega .
$$

Using now $\tan \phi=\dot{x}_{1} / \dot{x}_{4}$ and $\cos \phi=\dot{x}_{4} / \sqrt{1-\dot{x}_{2}^{2}}$, we can show that

$$
\dot{\phi}=\frac{d s}{d \nu} \frac{\dot{x}_{2}^{2}}{1-\dot{x}_{2}^{2}}\left(f_{1}^{\prime} f_{4}-f_{1} f_{4}^{\prime}\right)
$$

and a straightforward calculation gives

$$
\begin{aligned}
f_{1}^{\prime} f_{4}-f_{1} f_{4}^{\prime} & =2 \varphi^{\prime 2}+(\sinh \varphi)^{2}\left(1+\varphi^{\prime 2}\right)-\sinh \varphi \cosh \varphi \varphi^{\prime \prime} \\
& =2 \varphi^{\prime 2}-\lambda \sinh \varphi(\cosh \varphi)^{2}\left(1+\varphi^{\prime 2}\right)^{\frac{3}{2}}
\end{aligned}
$$

\footnotetext{
${ }^{38}$ In eqs. (B.25) and (B.26) the arguments of the various functions refer to the variable $s$.
} 
where in the last passage we have substituted the equations of motion in $\varphi^{\prime \prime}$. All in all, we get

$$
\dot{\phi}=\frac{d s}{d \nu} \frac{2 \varphi^{\prime 2}-\lambda \sinh \varphi(\cosh \varphi)^{2}\left(1+\varphi^{\prime 2}\right)^{\frac{3}{2}}}{(\cosh \varphi)^{2}\left(1+\varphi^{\prime 2}\right)-1} .
$$

Plugging this in eq. (B.27) and exploiting the symmetries of $\varphi(s)$ we obtain

$$
\Phi\left(\mathcal{C}_{\vec{u}}\right)=-2 \int_{\varphi_{0}}^{\tilde{\varphi}} d \varphi \frac{1}{\varphi^{\prime}} \frac{2 \varphi^{\prime 2}-\lambda \sinh \varphi(\cosh \varphi)^{2}\left(1+\varphi^{\prime 2}\right)^{\frac{3}{2}}}{\left[\cosh \varphi \sqrt{1+\varphi^{\prime 2}}\right]\left[(\cosh \varphi)^{2}\left(1+\varphi^{\prime 2}\right)-1\right]},
$$

and substituting the exact solution eq. (5.14)

$$
\sqrt{1+\varphi^{\prime 2}}=v(\varphi)=\frac{\cosh \varphi}{\frac{\lambda}{2}(f(\tilde{\varphi})-f(\varphi))} \equiv \frac{\cosh \varphi}{r(\varphi)}
$$

we finally obtain

$$
\Phi\left(\mathcal{C}_{\vec{u}}\right)=-2 \int_{\varphi_{0}}^{\tilde{\varphi}} d \varphi \frac{r(\varphi)\left[2 r(\varphi)\left((\cosh \varphi)^{2}-(r(\varphi))^{2}\right)-\lambda \sinh \varphi(\cosh \varphi)^{5}\right]}{(\cosh \varphi)^{2}\left[(\cosh \varphi)^{4}-(r(\varphi))^{2}\right] \sqrt{(\cosh \varphi)^{2}-(r(\varphi))^{2}}}
$$

\section{Spin-factor contribution in the large- $\lambda$ case}

The calculations of the previous appendix are exact, but in order to obtain an analytic expression for the spin-factor contribution we have to make some approximation. We consider therefore the case of large- $\lambda$, corresponding to small $\Delta=\tilde{\varphi}-\varphi_{0}$, in which case the calculation of the phase eq. (B.33) can be explicitly performed. Up to order $\mathcal{O}\left(\Delta^{2}\right)$ we have

$$
\Delta \simeq \frac{\theta}{2}, \quad \lambda \Delta \cosh \tilde{\varphi} \simeq \frac{\lambda \theta}{2} \cosh \tilde{\varphi} \simeq 1,
$$

so that setting $x=(\tilde{\varphi}-\varphi) / \Delta$, and expanding

$$
\begin{aligned}
\cosh \varphi & =\cosh \tilde{\varphi}-\Delta x \sinh \tilde{\varphi}+\mathcal{O}\left(\Delta^{2}\right) \\
r(\varphi) & =x(\cosh \tilde{\varphi}-\Delta x \sinh \tilde{\varphi})+\mathcal{O}\left(\Delta^{2}\right)
\end{aligned}
$$

we obtain to leading order

$$
\Phi\left(\mathcal{C}_{\vec{u}}\right)=2 \int_{0}^{1} d x \frac{x}{\sqrt{1-x^{2}}} \frac{\sinh \tilde{\varphi}}{(\cosh \tilde{\varphi})^{2}-x^{2}}+\mathcal{O}(\Delta)=\pi-2 \arctan \sinh \tilde{\varphi}+\mathcal{O}(\theta) .
$$

Consistently with what we have done in the calculation of the effective action, we neglect higher-order terms when performing the analytic continuation to Minkowski space-time, and since $\tilde{\varphi}$ does not depend on $\theta$ in the given approximation, $\sinh \tilde{\varphi}=\sqrt{\left(b_{c} / b\right)^{2}-1}$, we have

$$
\Phi^{(M)}\left(\mathcal{C}_{\vec{u}}\right)=\left.\Phi\left(\mathcal{C}_{\vec{u}}\right)\right|_{\theta \rightarrow-i \chi}=\pi-2 \arctan \left(\sqrt{\left(\frac{b_{c}}{b}\right)^{2}-1}\right) .
$$

Therefore, the quantity $\Phi^{(M)}\left(\mathcal{C}_{\vec{u}}\right)$ remains real in the region $b \leq b_{c}$ after the analytic continuation to Minkowski space. 
At this point, in order to go to $b>b_{c}$ we have to perform a second analytic continuation, which according to the " $-i \varepsilon$ " prescription leads to

$$
\Phi^{(M)}\left(\mathcal{C}_{\vec{u}}\right) \underset{b>b_{c}}{\rightarrow}=\pi-\frac{2}{i} \log \left[\frac{b}{b_{c}}\left(1+\sqrt{1-\left(\frac{b_{c}}{b}\right)^{2}}\right)\right],
$$

where we have used the representation

$$
\arctan x=\frac{1}{2 i} \log \frac{1+i x}{1-i x}
$$

for the arctangent. Exponentiating this expression we obtain

$$
e^{ \pm i \Phi^{(M)}\left(\mathcal{C}_{\vec{u}}\right)} \underset{b>b_{c}}{\rightarrow}=-\left(\frac{b}{b_{c}}\right)^{2}\left(1 \mp \sqrt{1-\left(\frac{b_{c}}{b}\right)^{2}}\right)^{2} .
$$

However, a comparison of the analytic result eq. (C.4) with numerical calculations for the exact expression eq. (B.33) shows that, although there is good agreement for small values of $b / b_{c}$, the distance between analytic and numerical results increases as $b$ tends to $b_{c}$. In contrast, the same comparison for the Euclidean effective action shows that the distance between analytic and numerical results decreases as $b$ tends to $b_{c}$. For this reason, the extrapolation eq. (C.5) of $\Phi^{(M)}\left(\mathcal{C}_{\vec{u}}\right)$ to $b>b_{c}$ cannot be used, and we have thus preferred not to include this result in the main analysis of the present work, delaying a more careful study to a future publication.

Open Access. This article is distributed under the terms of the Creative Commons Attribution Noncommercial License which permits any noncommercial use, distribution, and reproduction in any medium, provided the original author(s) and source are credited.

\section{References}

[1] A.M. Polyakov, String theory and quark confinement, Nucl. Phys. Proc. Suppl. 68 (1998) 1 [hep-th/9711002] [INSPIRE].

[2] J.M. Maldacena, The Large- $N$ limit of superconformal field theories and supergravity, Adv. Theor. Math. Phys. 2 (1998) 231 [Int. J. Theor. Phys. 38 (1999) 1133] [hep-th/9711200] [INSPIRE].

[3] S. Gubser, I.R. Klebanov and A.M. Polyakov, Gauge theory correlators from noncritical string theory, Phys. Lett. B 428 (1998) 105 [hep-th/9802109] [INSPIRE].

[4] E. Witten, Anti-de Sitter space and holography, Adv. Theor. Math. Phys. 2 (1998) 253 [hep-th/9802150] [INSPIRE].

[5] G. Policastro, D. Son and A. Starinets, The Shear viscosity of strongly coupled $N=4$ supersymmetric Yang-Mills plasma, Phys. Rev. Lett. 87 (2001) 081601 [hep-th/0104066] [INSPIRE].

[6] R.A. Janik and R.B. Peschanski, Asymptotic perfect fluid dynamics as a consequence of AdS/CFT, Phys. Rev. D 73 (2006) 045013 [hep-th/0512162] [INSPIRE]. 
[7] A. Bernamonti and R. Peschanski, Time-dependent AdS/CFT correspondence and the quark-gluon plasma, Nucl. Phys. Proc. Suppl. 216 (2011) 94 [arXiv:1102.0725] [InSPIRE].

[8] E. Witten, Anti-de Sitter space, thermal phase transition and confinement in gauge theories, Adv. Theor. Math. Phys. 2 (1998) 505 [hep-th/9803131] [INSPIRE].

[9] J. Polchinski and M.J. Strassler, Hard scattering and gauge/string duality, Phys. Rev. Lett. 88 (2002) 031601 [hep-th/0109174] [INSPIRE].

[10] A. Karch, E. Katz, D.T. Son and M.A. Stephanov, Linear confinement and AdS/QCD, Phys. Rev. D 74 (2006) 015005 [hep-ph/0602229] [INSPIRE].

[11] R. Janik and R.B. Peschanski, High-energy scattering and the AdS/CFT correspondence, Nucl. Phys. B 565 (2000) 193 [hep-th/9907177] [INSPIRE].

[12] R. Janik and R.B. Peschanski, Minimal surfaces and Reggeization in the AdS/CFT correspondence, Nucl. Phys. B 586 (2000) 163 [hep-th/0003059] [INSPIRE].

[13] R.A. Janik, String fluctuations, AdS/CFT and the soft Pomeron intercept, Phys. Lett. B 500 (2001) 118 [hep-th/0010069] [INSPIRE].

[14] R. Janik and R.B. Peschanski, Reggeon exchange from AdS/CFT, Nucl. Phys. B 625 (2002) 279 [hep-th/0110024] [INSPIRE].

[15] M. Giordano and R. Peschanski, High Energy Bounds on Soft $N=4$ SYM Amplitudes from AdS/CFT, JHEP 05 (2010) 037 [arXiv: 1003.2309] [inSPIRE].

[16] P.D.B. Collins, An Introduction to Regge Theory and High-Energy Physics, Cambridge University Press, Cambridge U.K. (1977).

[17] O. Nachtmann, Considerations concerning diffraction scattering in quantum chromodynamics, Annals Phys. 209 (1991) 436 [INSPIRE].

[18] H.G. Dosch, E. Ferreira and A. Kramer, Nonperturbative QCD treatment of high-energy hadron hadron scattering, Phys. Rev. D 50 (1994) 1992 [hep-ph/9405237] [INSPIRE].

[19] E. Meggiolaro, A Remark on the high-energy quark quark scattering and the eikonal approximation, Phys. Rev. D 53 (1996) 3835 [hep-th/9506043] [INSPIRE].

[20] O. Nachtmann, High-energy collisions and nonperturbative QCD, in Perturbative and nonperturbative aspects of quantum field theory, proceedings of the 35th International University School Of Nuclear And Particle Physics, Schladming Austria (1996), H. Latal and W. Schweiger eds., Springer-Verlag, Heidelberg Germany (1997) [hep-ph/9609365] [INSPIRE].

[21] E.R. Berger and O. Nachtmann, Differential cross-sections for high-energy elastic hadron hadron scattering in nonperturbative QCD, Eur. Phys. J. C 7 (1999) 459 [hep-ph/9808320] [INSPIRE].

[22] H.G. Dosch, in At the frontier of Particle Physics - Handbook of QCD (Boris Ioffe Festschrift). Vol. 2, M. Shifman eds., World Scientific, Singapore (2001), pp. 1195-1236.

[23] E. Meggiolaro, Eikonal propagators and high-energy parton parton scattering in gauge theories, Nucl. Phys. B 602 (2001) 261 [hep-ph/0009261] [INSPIRE].

[24] A. Shoshi, F. Steffen and H. Pirner, $S$ matrix unitarity, impact parameter profiles, gluon saturation and high-energy scattering, Nucl. Phys. A 709 (2002) 131 [hep-ph/0202012] [INSPIRE]. 
[25] A. Shoshi, F. Steffen, H.G. Dosch and H. Pirner, Confining QCD strings, Casimir scaling and a Euclidean approach to high-energy scattering, Phys. Rev. D 68 (2003) 074004 [hep-ph/0211287] [INSPIRE].

[26] E.V. Shuryak and I. Zahed, Instanton induced effects in QCD high-energy scattering, Phys. Rev. D 62 (2000) 085014 [hep-ph/0005152] [INSPIRE].

[27] M. Giordano and E. Meggiolaro, High-energy hadron-hadron (dipole-dipole) scattering from lattice QCD, Phys. Rev. D 78 (2008) 074510 [arXiv:0808.1022] [InSPIRE].

[28] M. Giordano and E. Meggiolaro, Instanton effects on Wilson-loop correlators: a new comparison with numerical results from the lattice, Phys. Rev. D 81 (2010) 074022 [arXiv: 0910.4505] [inSPIRE].

[29] R.C. Brower, J. Polchinski, M.J. Strassler and C.-I. Tan, The Pomeron and gauge/string duality, JHEP 12 (2007) 005 [hep-th/0603115] [INSPIRE].

[30] R.C. Brower, M.J. Strassler and C.-I. Tan, On the eikonal approximation in AdS space, JHEP 03 (2009) 050 [arXiv:0707.2408] [INSPIRE].

[31] R.C. Brower, M.J. Strassler and C.-I. Tan, On The Pomeron at Large 't Hooft Coupling, JHEP 03 (2009) 092 [arXiv:0710.4378] [InSPIRE].

[32] R.C. Brower, M. Djuric and C.-I. Tan, Odderon in gauge/string duality, JHEP 07 (2009) 063 [arXiv:0812.0354] [INSPIRE].

[33] R. Brower, M. Djuric and C.-I. Tan, Elastic and Diffractive Scattering after AdS/CFT, arXiv:0911. 3463 [INSPIRE].

[34] L. Cornalba, M.S. Costa, J. Penedones and R. Schiappa, Eikonal Approximation in AdS/CFT: From Shock Waves to Four-Point Functions, JHEP 08 (2007) 019 [hep-th/0611122] [INSPIRE].

[35] L. Cornalba, M.S. Costa, J. Penedones and R. Schiappa, Eikonal Approximation in AdS/CFT: Conformal Partial Waves and Finite N Four-Point Functions, Nucl. Phys. B 767 (2007) 327 [hep-th/0611123] [INSPIRE].

[36] L. Cornalba, M.S. Costa and J. Penedones, Eikonal approximation in AdS/CFT: Resumming the gravitational loop expansion, JHEP 09 (2007) 037 [arXiv:0707.0120] [INSPIRE].

[37] L. Cornalba, Eikonal methods in AdS/CFT: Regge theory and multi-reggeon exchange, arXiv: 0710.5480 [INSPIRE].

[38] L. Cornalba, M.S. Costa and J. Penedones, Eikonal Methods in AdS/CFT: BFKL Pomeron at Weak Coupling, JHEP 06 (2008) 048 [arXiv:0801.3002] [INSPIRE].

[39] J.L. Albacete, Y.V. Kovchegov and A. Taliotis, DIS on a Large Nucleus in AdS/CFT, JHEP 07 (2008) 074 [arXiv:0806.1484] [INSPIRE].

[40] A. Taliotis, DIS from the AdS/CFT correspondence, Nucl. Phys. A 830 (2009) 299C-302C [arXiv: 0907.4204] [INSPIRE].

[41] A. Mueller, A. Shoshi and B.-W. Xiao, Deep inelastic and dipole scattering on finite length hot $N=4$ SYM matter, Nucl. Phys. A 822 (2009) 20 [arXiv:0812.2897] [INSPIRE].

[42] E. Levin and I. Potashnikova, Soft interaction at high energy and $N=4 S Y M$, JHEP 06 (2009) 031 [arXiv: 0902.3122] [INSPIRE].

[43] D. Kharzeev and E. Levin, D-instantons and multiparticle production in $N=4 S Y M$, JHEP 01 (2010) 046 [arXiv:0910.3355] [INSPIRE]. 
[44] E. Avsar, Y. Hatta and T. Matsuo, Odderon in baryon-baryon scattering from the AdS/CFT correspondence, JHEP 03 (2010) 037 [arXiv:0912.3806] [INSPIRE].

[45] Y. Makeenko, Effective String Theory and QCD Scattering Amplitudes, Phys. Rev. D 83 (2011) 026007 [arXiv: 1012.0708] [InSPIRE].

[46] R.A. Brandt, F. Neri and D. Zwanziger, Lorentz invariance from classical particle paths in quantum field theory of electric and magnetic charge, Phys. Rev. D 19 (1979) 1153 [INSPIRE].

[47] R.A. Brandt, A. Gocksch, M. Sato and F. Neri, Loop space, Phys. Rev. D 26 (1982) 3611 [INSPIRE].

[48] A.M. Polyakov, Fermi-Bose Transmutations Induced by Gauge Fields, Mod. Phys. Lett. A 3 (1988) 325 [inSPIRE].

[49] G. Korchemsky, Quantum geometry of the Dirac fermions, Phys. Lett. B 232 (1989) 334 [INSPIRE].

[50] G. Korchemsky, Quantum geometry of Dirac fermions, Int. J. Mod. Phys. A 7 (1992) 339 [INSPIRE].

[51] E. Meggiolaro, The High-energy quark quark scattering: From Minkowskian to Euclidean theory, Z. Phys. C 76 (1997) 523 [hep-th/9602104] [inSPIRE].

[52] E. Meggiolaro, The Analytic continuation of the high-energy quark quark scattering amplitude, Eur. Phys. J. C 4 (1998) 101 [hep-th/9702186] [inSPIRE].

[53] E. Meggiolaro, The Analytic continuation of the high-energy parton-parton scattering amplitude with an IR cutoff, Nucl. Phys. B 625 (2002) 312 [hep-ph/0110069] [INSPIRE].

[54] E. Meggiolaro, On the loop-loop scattering amplitudes in Abelian and non-Abelian gauge theories, Nucl. Phys. B 707 (2005) 199 [hep-ph/0407084] [INSPIRE].

[55] M. Giordano and E. Meggiolaro, Analyticity and crossing symmetry of the eikonal amplitudes in gauge theories, Phys. Rev. D 74 (2006) 016003 [hep-ph/0602143] [INSPIRE].

[56] E. Meggiolaro, Can the Pomeron be derived from a Euclidean-Minkowskian duality?, Phys. Lett. B 651 (2007) 177 [hep-ph/0612307] [INSPIRE].

[57] M. Giordano and E. Meggiolaro, A Nonperturbative foundation of the Euclidean-Minkowskian duality of Wilson-loop correlation functions, Phys. Lett. B 675 (2009) 123 [arXiv:0902.4145] [INSPIRE].

[58] L.F. Alday and J.M. Maldacena, Gluon scattering amplitudes at strong coupling, JHEP 06 (2007) 064 [arXiv: 0705.0303] [INSPIRE].

[59] S.G. Naculich and H.J. Schnitzer, Regge behavior of gluon scattering amplitudes in $N=4$ SYM theory, Nucl. Phys. B 794 (2008) 189 [arXiv: 0708.3069] [InSPIRE].

[60] M. Giordano, R. Peschanski and S. Seki, $\mathcal{N}=4$ SYM Regge Amplitudes and Minimal Surfaces in AdS/CFT Correspondence, arXiv:1110.3680 [INSPIRE].

[61] J.M. Maldacena, Wilson loops in large-N field theories, Phys. Rev. Lett. 80 (1998) 4859 [hep-th/9803002] [INSPIRE].

[62] S.-J. Rey and J.-T. Yee, Macroscopic strings as heavy quarks in large- $N$ gauge theory and anti-de Sitter supergravity, Eur. Phys. J. C 22 (2001) 379 [hep-th/9803001] [INSPIRE]. 
[63] D.J. Gross and H. Ooguri, Aspects of large- $N$ gauge theory dynamics as seen by string theory, Phys. Rev. D 58 (1998) 106002 [hep-th/9805129] [INSPIRE].

[64] N. Drukker, D.J. Gross and H. Ooguri, Wilson loops and minimal surfaces, Phys. Rev. D 60 (1999) 125006 [hep-th/9904191] [INSPIRE].

[65] A. Brandhuber, N. Itzhaki, J. Sonnenschein and S. Yankielowicz, Wilson loops, confinement and phase transitions in large- $N$ gauge theories from supergravity, JHEP 06 (1998) 001 [hep-th/9803263] [INSPIRE].

[66] Y. Kinar, E. Schreiber and J. Sonnenschein, $Q$ anti- $Q$ potential from strings in curved space-time: Classical results, Nucl. Phys. B 566 (2000) 103 [hep-th/9811192] [INSPIRE].

[67] Y. Kinar, E. Schreiber, J. Sonnenschein and N. Weiss, Quantum fluctuations of Wilson loops from string models, Nucl. Phys. B 583 (2000) 76 [hep-th/9911123] [INSPIRE].

[68] P.A. Frampton, Dual resonance models and superstrings, World Scientific, Singapore (1986).

[69] M. Giordano, Wilson-loop formalism for Reggeon exchange in soft high-energy scattering, to appear.

[70] A. Armoni, Beyond The Quenched (or Probe Brane) Approximation in Lattice (or Holographic) QCD, Phys. Rev. D 78 (2008) 065017 [arXiv:0805.1339] [INSPIRE].

[71] M. Giordano and R. Peschanski, Reggeon exchange from Gauge/Gravity duality: loop corrections and unitarity, work in progress.

[72] U. Dierkes, S. Hildebrandt, A. Küster and O. Wohlrab, Minimal Surfaces I: Boundary Value Problems, Springer-Verlag, Berlin Germany (1992).

[73] M. Froissart, Asymptotic behavior and subtractions in the Mandelstam representation, Phys. Rev. 123 (1961) 1053 [INSPIRE].

[74] A. Martin, Extension of the axiomatic analyticity domain of scattering amplitudes by unitarity. I, Nuovo Cim. A 42 (1966) 930.

[75] L. Łukaszuk and A. Martin, Absolute upper bounds for $\pi \pi$ scattering, Nuovo Cim. A 52 (1967) 122.

[76] M. Abramowitz and I. A. Stegun, Handbook of Mathematical Functions with Formulas, Graphs, and Mathematical tables, Dover, New York U.S.A. (1964).

[77] I. Korchemskaya and G. Korchemsky, Polyakov spin factor, Berry phase and random walk of spinning particles, J. Phys. A 24 (1991) 4511 [inSPIRE]. 